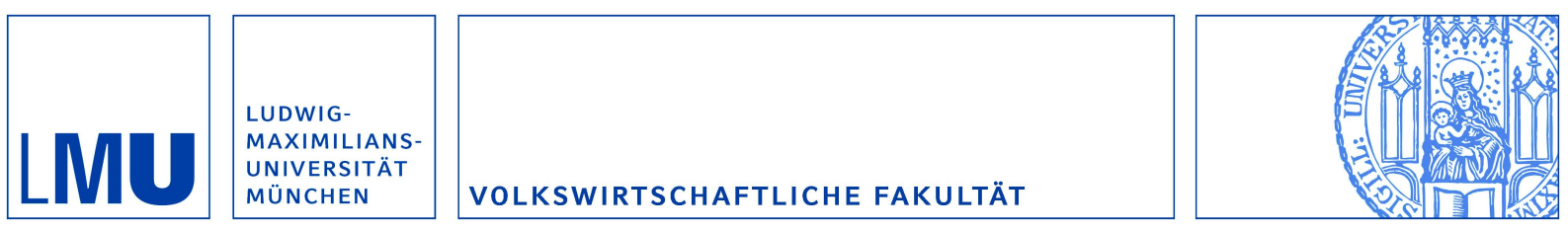

Meya, Johannes; Poutvaara, Panu und Schwager, Robert:

Pocketbook Voting, Social Preferences, and Expressive Motives in Referenda

Munich Discussion Paper No. 2017-12

Department of Economics

University of Munich

Volkswirtschaftliche Fakultät

Ludwig-Maximilians-Universitäł München

Online at https://doi.org/10.5282/ubm/epub.38425 


\title{
Pocketbook Voting, Social Preferences, and Expressive Motives in Referenda*
}

\author{
Johannes Meya $^{\dagger} \quad$ Panu Poutvaara ${ }^{\ddagger} \quad$ Robert Schwager ${ }^{\S}$
}

\begin{abstract}
May 2017
Abstract

We develop and test a theory of voting and turnout decisions that integrates self-interest, social preferences, and expressive motives. Our model implies that if pocketbook benefits are relevant, voters either perceive their impact on the outcome to be non-negligible, or expressive motivations do not play a role in the decision on how to vote. Conversely, if own pocketbook benefits do not explain voting, then voting is expressive. If the perceived probability of being pivotal is non-negligible, social preferences and expressive concerns are observationally equivalent. Our empirical analysis studies collective choices which are analogous to decisions on local public goods. We consider referenda among university students on whether to collectively purchase deeply discounted flat rate tickets for public transportation and cultural amenities. Individual usage data allow quantifying the monetary benefits associated with each ticket. As voters had precise information on the individual costs and benefits, our setting comprises a real-world laboratory of direct democracy. We find that monetary benefits strongly influence participation and voting. However, social or expressive motives, such as stated altruism, environmental concerns, and paternalism, are decisive for a significant minority. Our results rule out purely expressive voting and imply that a substantial share of the electorate perceived their impact on the outcome to be non-negligible.
\end{abstract}

Keywords: pocketbook voting, social preferences, expressive voting, instrumental voting, public goods, altruism, referendum

JEL classification: D72, H41, D64

*This is a thoroughly revised and substantially extended version of CESifo Working Paper 5267, entitled 'Pocketbook voting and social preferences in referenda'. We thank Thushyanthan Baskaran, Mikael Elinder, Ingo Geishecker, Arye Hillman, Henrik Jordahl, Martin Kocher, Niklas Potrafke, Andrei Shleifer, and participants in several seminars and conferences for helpful comments, Felix Albrecht for research assistance, and everyone who helped with the data collection. Johannes Meya and Robert Schwager gratefully acknowledge financial support from the German Federal Ministry of Education and Research under grant number 01PW11004. Responsibility for the content remains with the authors.

${ }^{\dagger}$ Bundesnetzagentur, Bonn, Germany, jmeya@gwdg.de. The author primarily wrote this paper when he was doctoral student and postdoctoral researcher at University of Goettingen. The paper is written in the author's private capacity, is exclusively the author's responsibility, and does not in any way reflect the position of Bundesnetzagentur.

${ }^{\ddagger}$ LMU Munich, Ifo Institute - Leibniz Institute for Economic Research at the University of Munich, CESifo and IZA, Poschingerstrasse 5, Germany - 81679 Munich, poutvaara@ifo.de

${ }^{\S}$ University of Goettingen, Department of Economics, Platz der Goettinger Sieben 3, Germany - 37073 Goettingen, rschwag@uni-goettingen.de 


\section{Introduction}

Referenda are an integral part of democracy in several jurisdictions, such as California and Switzerland. Prominent examples include Proposition 13 in 1978 which decisively shaped local public finances in California by bounding the property tax rate (see California State Board of Equalization, 2012, p. 1) and the referendum on the Gotthard train tunnel in Switzerland in 1992. In recent years, other countries such as Germany, Italy, and the United Kingdom have also increasingly held referenda.

Despite their increasing popularity, referenda are controversial. Proponents welcome the broad public debate and the democratic legitimacy of decisions ensured by referenda. In this spirit, Rousseau (2012 [1762], p. 65) argued 250 years ago that "Every law the people has not ratified in person is null and void - is, in fact, not a law." Opponents fear that uninformed or ideologically biased citizens either do not bother to vote or make inefficient or inequitable decisions. Schumpeter (1994 [1942], p. 261) was convinced that "[the private citizen] expends less disciplined effort on mastering a political problem than he expends on a game of bridge."

We contribute to this debate by theoretically and empirically analyzing the motives for participating in a referendum and for voting against or in favor of a proposal. Our set-up is particularly tailored to public policy decisions at the local level, where referenda are especially frequent. The importance of local direct democracy was already highlighted by De Tocqueville (1981 [1835], p. 124-127), who emphasized that citizens can and should be trusted to decide independently on their own affairs. In the same spirit, Tiebout (1956) shows that independent local jurisdictions take efficient decisions on local public goods as long as there are no external effects. By analyzing voters' motives in such decisions, we contribute to the understanding of local democracy.

We propose a simple theoretical model describing the decisions on whether and how to vote on a tax-financed provision of a public good. The motives we consider are 'pocketbook voting', social preferences, and expressive concerns. Pocketbook voting refers to voting along own monetary interests. The latter two motives relate to how the issue on which the vote takes is seen related to others, but differ in one crucial aspect. Agents with social preferences want to affect the outcome of the vote, for example because they have altruistic or paternalistic feelings towards other members of society, or because they care for the common good. In this sense, social preferences, like pocketbook motives, are instrumental. In contrast, expressive voters derive utility from the act of voting in a way which is in line with what they think would be socially good or morally just, independently of whether their vote affects the actual outcome. 
Using this model, we characterize the individual voting decision as a function of the size of monetary gains or losses conferred by the public good, the strength of social or expressive motives, and the perceived probability of changing the referendum outcome. When expressive motives are relevant, they will dominate the voting decision as long as the voter considers the probability of being pivotal to be negligible. Conversely, in the presence of expressive concerns, pocketbook benefits affect the voting decision only if voters put a sufficiently high estimate on this probability. If this is the case, however, voters also expect to affect the outcome for other members of society. Hence, when pocketbook voting is observed, an empirical finding that voters care about common goods or benefits of others cannot unequivocally be attributed to expressive voting, but may as well reflect social preferences, or both motives.

To sum up, our theoretical model implies that if voters consider the probability of being pivotal to be strictly positive, social preferences and expressive motives are observationally equivalent. Therefore, expressive voting can be shown to exist only in the case where pocketbook voting is not observed: if voters do not react to pocketbook benefits they must believe that their influence on the outcome of the vote is negligible, and any concern for common goods or benefits of others must therefore be expressive.

When there are no expressive motives, we show that pocketbook voting affects the voting decision even when the probability of being pivotal is arbitrarily small. This is because in the absence of expressive voting, all remaining benefits of voting are instrumental, and hence weighted down with the same probability. As a consequence, a theory without expressive motives is consistent with empirically observing pocketbook voting without placing restrictions on the subjective probability of affecting the outcome.

The decision to participate in the referendum is driven by the same variables as the voting decision together with benefits and costs which are unrelated to how one votes, such as a civic duty motive for voting and time cost to cast one's ballot. We show that when expressive motives are relevant, abstention can be a rational choice even when voting is costless. This arises when monetary benefits and expressive concerns suggest different voting decisions, for example when the individual gains in monetary terms from the public good but thinks that others lose from it. Then, abstaining is a cheaper way of satisfying expressive motives than voting against one's own pocketbook interest.

We test our theory with the help of referenda on flat rate tickets for students at University of Goettingen in Germany. If passed, these tickets give all students the right to unlimited use of a facility, such as public transportation or cultural amenities. The price of each ticket is very attractive compared with that for indi- 
vidual purchases; however, buying a ticket becomes compulsory for every student if the majority vote in favor. These tickets therefore share essential features with tax-financed public projects or local public goods. Collectively procuring a ticket reduces the per capita cost of the respective facility so that frequent users stand to gain substantially from approval by the majority. At the same time, some voters use the facility in question very little or not at all but are still forced to pay as much for it as anyone else.

Investigating these referenda is particularly promising because they involve easyto-understand public policy decisions. In particular, in our setting, the voters knew exactly what a ticket would cost, and the benefits were clearly defined. This setting fits particularly well to local democracy in an independent, self-financed jurisdiction in the sense of Tiebout (1956). In fact, the student body is quite similar to the citizens of a small jurisdiction, who decide democratically on a public good which is used and financed exclusively by them. In contrast, if the vote had involved, for example, a large infrastructure project, then the costs and benefits would have been more widespread and uncertain. Different voting decisions could also then reflect different subjective expectations for possible deviations from projections and differences in risk attitudes. In this sense, the referenda that we study are like a 'laboratory' for direct democratic decisions, where confounding influences are reduced to a minimum.

Our first dataset is on a referendum regarding a ticket for regional trains held in 2010. This dataset was collected online. It also encompasses students who did not participate in the referendum, allowing for an analysis of the decision whether to vote. The second dataset covers votes on tickets for regional trains, cultural facilities, and local buses held in 2013. It was collected with a paper-based exit poll, therefore being restricted to voters.

Each dataset contains detailed information on usage behavior, votes, political preferences and other characteristics of over a thousand respondents. In the first dataset, we construct a monetary measure of the individual benefits conferred by the ticket. To do so, we combine information on the number of trips to visit parents with regular ticket prices that we derive from parental address data. Subtracting the price of the flat rate ticket yields a measure of the net monetary savings an individual student obtains from the ticket. An attractive feature of this measure arises from the fact that the ticket in question covered only rail tracks served by one company, while tracks served by other operators were not included. Hence, residence of parents on either subset of tracks induces a quasi-random variation which is unrelated to the variation created by distance to the university. In the second dataset, usage is reported in categorical variables.

Additionally, students were asked the extent to which the interests of others 
shaped their voting decisions. Other motives included social goals, such as promoting local cultural life, and expressing protest against the pricing policy of the railway company. These variables capture social preferences and expressive motives of students.

Our primary focus is on the probability of voting in favor of these tickets. We find strong evidence for pocketbook voting. Most students vote in line with their monetary interests. In the first dataset, among those who gain from the train ticket, a 10 percent increase in net savings raises the probability of voting in favor by 0.6 to 0.7 percentage points. This finding translates into widely differing predictions, given that positive net savings vary between zero and more than three thousand euros per year. Also among those who benefit less from the ticket than it costs, a lower net loss tends to increase the probability to support the ticket, even though the results are mostly not statistically significant. In the second dataset, a student who uses a facility very often is between 52 and 77 percentage points more likely to vote in favor of the corresponding ticket than a student who never uses it.

However, our results show that monetary self-interest is not the entire story. Variables capturing social preferences or expressive motives, such as stated altruism and merit good considerations, show highly significant and economically relevant effects. A sizable share of students who do not stand to gain from a ticket in monetary terms vote in favor because of such motives. The analysis suggests that these motives were likely pivotal in one of the four referenda and close to being pivotal in another. Together with pocketbook voting, they can rationalize almost all votes cast. In contrast, party preferences are mostly not relevant to the voting decision.

Based on our theory, the presence of pocketbook voting means that we cannot distinguish whether the significance of the variables related to other students or public good considerations stems from social preferences, expressive motives, or both. However, our theory also implies that if monetary benefits affect the voting decision then voters either perceive the probability to change the outcome to be nonnegligible, or expressive motives are absent. From this we conclude that observed behavior is inconsistent with a theory of purely expressive voting.

In the last part of the paper, the dependent variable is participation in the referendum. We find that among those who gain from the ticket, the probability of taking part increases with net savings. This result suggests that a substantial share of the electorate perceived their impact on the outcome to be non-negligible. Stated altruism is also found to affect the participation decision, albeit in a specific way. Students who expect that their preferred outcome would cause losses to others and, at the same time, mention concern for others are less likely to participate. A possible explanation for this finding is that abstaining is an attractive compromise 
when own monetary gains and expressive concerns for others call for different voting decisions.

The remainder of the paper is structured as follows. In Section 2, we relate our work to the literature on voting motives. Section 3 contains the theoretical model on which we base our empirical work. Section 4 presents the data and some background information. In Section 5, we give a descriptive overview of voting and participation motives. Econometric results on voting and participation decisions are presented in Sections 6 and 7, respectively. Section 8 concludes the paper.

\section{Related literature}

Our paper contributes to the literature on the motives of voters. A central question in this literature is the extent to which citizens vote according to their narrowly defined self-interest and to which voting decisions are driven by social considerations or expressive motives. In addition, research has investigated whether such motives determine the participation decision. Ours is the first paper that analyzes both the decisions whether to vote and how to vote in the presence of self-interest and social considerations.

Pocketbook voting is the starting point in models of income redistribution that build on Meltzer and Richard (1981), in the theory of probabilistic voting (Lindbeck and Weibull, 1993; Dixit and Londregan, 1996) and in the median voter models of local public finance (Romer et al., 1992; Epple and Romano, 1996; Epple et al., 2001). The empirical literature on economic voting has mostly focused on representative democracy. Lewis-Beck and Stegmaier (2007) survey more than 400 studies without finding much evidence for pocketbook voting. Most of these studies suffer from severe identification problems: they assume that voters attribute all changes in their financial situation to the policies of the incumbent government. When analyzing well defined past policies, Levitt and Snyder (1997) and Manacorda et al. (2011) find that voters increase their support for the government if they have benefited from its transfers in the past. Elinder et al. (2015) find strong evidence for prospective pocketbook voting: voters already react when reforms appear as electoral promises.

Several authors specifically seek to empirically detect pocketbook motives in referenda. In an early contribution, Deacon and Shapiro (1975) find that voters in cities connected to the Bay Area Rapid Transit (BART) system were more likely to favor a proposition that would shift gas tax revenues to public transportation. Similarly, voters in precincts that are located near sports stadiums feel more positively toward subsidizing them (Coates and Humphreys, 2006). Intriguingly, according to Potrafke (2013), this finding does not hold for concert halls. Vlachos (2004) concludes that voting patterns in the Swedish referendum on EU membership are in 
line with conflicting regional interests. These studies are based on voting results at regional or local level, while we analyze voting at individual level.

Pocketbook voting has been challenged by the theory of expressive voting, proposed by Tullock (1971) and Brennan and Buchanan (1984) and further developed by Brennan and Hamlin (1998), Hillman (2010), and Hamlin and Jennings (2011). This theory maintains that voters perceive their probability of being pivotal to be negligible, and that they derive utility from voting in a particular way irrespective of the outcome. In this view, a voter who supports a proposal which benefits other members of society does not aim at actually raising others' utility, as social preferences would imply, but wants to sustain an image as a socially-minded person at low cost.

Social preferences and expressive motives have to date been neglected in the empirical analysis of real world referenda. This neglect contrasts with experimental studies on voting behavior that typically find that in addition to monetary selfinterest, voting reflects various types of social preferences. In particular, Tyran and Sausgruber (2006) show that inequity aversion in the sense of Fehr and Schmidt (1999) is important in laboratory elections. Introducing a novel random price voting mechanism, Messer et al. (2010) conclude that subjects' behavior is better explained by pure altruism than by inequity aversion. Balafoutas et al. (2013) find that while voting on redistribution is mostly predicted by self-interest, there is greater support for redistribution when inequalities are arbitrary than when they reflect performance in an experimental task. This finding is in line with the theoretical insights in Piketty (1995) as well as in Bénabou and Tirole (2006) and the conclusions derived by Fong (2001) and Alesina and Angeletos (2005) from survey evidence.

Experimental evidence on expressive voting is mixed. Several studies confront subjects with a vote between an own monetary payout and a gift to charity. In such a setting, Carter and Guerette (1992) and Fischer (1996) exogenously vary the probability that the individual's choice is decisive. While this has very limited impact on support of the charitable option in the experiment conducted by Carter and Guerette (1992), Fischer (1996) finds that subjects are more likely to support charity if they anticipate not to affect the outcome. Tyran (2004) allows subjects who voted against the proposal to withhold their contribution to charity when the proposal passes. He does not find any difference in behavior between this treatment and the low-cost situation where the contribution is almost surely independent of the individual vote, contradicting expressive voting.

In general, it should be noted that pocketbook voting does not exclude taking into account what is good for others, or expressing a concern for others. Fiorina (1978) and Markus (1988) conclude that both self-interest and convictions on what benefits society matter to American voters. In Sweden, most survey respondents 
admitted that their own interest mattered either as much as or more than their convictions regarding what benefits society when they chose which party to vote for (Carlsson and Johansson-Stenman, 2010).

We also contribute to the literature on voter turnout. Downs (1957) as well as Riker and Ordeshook (1968) already presented the idea that the more that is at stake, the more likely an individual is to vote. Indeed, Andersen et al. (2014) observe that turnout in Norwegian local elections is higher in jurisdictions with high hydropower income. Alternative explanations suggest that voting is driven by ethical concerns (Harsanyi, 1980; Coate and Conlin, 2004), social pressure (Gerber et al., 2008; Funk, 2010), expressive motivations (Brennan and Buchanan, 1984; Brennan and Hamlin, 1998; Coate et al., 2008; Hillman, 2010), or social identity (Akerlof and Kranton, 2000; Ben-Bassat and Dahan, 2012; Hillman et al., 2015).

Finally, our paper is also broadly related to the literature that investigates the impact of direct democracy on the public budget. Referenda are associated with lower public spending in both the US (Matsusaka, 1995) and Switzerland (Feld and Kirchgässner, 2001; Feld and Matsusaka, 2003; Funk and Gathmann, 2011). The focus of our paper differs from these contributions in that we do not aim to compare direct and representative democracy. Nevertheless, our finding that those who benefit most are most likely to vote suggests that direct democracy does not necessarily result in underspending.

Extending previous literature, our paper enhances both the theoretical and the empirical understanding of the motives for voting. We propose a theoretical model of the decisions whether and how to vote which integrates pocketbook motives, instrumental social preferences and expressive concerns. Empirically, we find evidence for pocketbook voting and show that the likelihood of participating increases with the personal stake. These results are broadly in line with the contributions mentioned above. However, we go further by analyzing individual voting decisions in referenda rather than relying on regional vote shares. Moreover, we study the role of social preferences and expressive concerns in real world referenda. This complements existing literature on such motives which to date has mostly been confined to laboratory experiments.

\section{Theoretical framework}

We present a simple theoretical framework on how voters decide whether to vote in a referendum, and then how to vote, provided that they decide to vote. We solve the model backwards, analyzing first the decision on how to vote, conditional on voting. Voting takes place on whether to collectively purchase a public good. If the proposal is passed, all members of society get access to the public good and have to 
pay for it. We denote the case in which the public good is passed by superscript 1 , and the case in which the public good is not passed by superscript 0 .

\subsection{How to vote if voting}

The utility of voters is driven by pocketbook benefits, social preferences, and expressive motivations. Pocketbook benefits arise from the individual's use of the public good and the monetary cost he or she has to pay for it, and relate to the outcome of the vote. Social preferences relate to how the voter values the effects of the voting outcome on other people. They include the valuation of the benefits which other members of the polity enjoy when the public good is provided, the quality of local culture, the public transportation system, and the environment. Expressive motivations are based on personal utility from the act of voting in a certain way, independently on whether this affects the outcome of the vote. We describe these components of utility one after the other, starting with pocketbook benefits.

In the context of the collectively purchased tickets we analyze, providing the public good amounts to granting free and unlimited use of some facility, financed by a compulsory fee $t$ collected from every member of society. In contrast, if the public good is not provided, individuals have to pay a price $\tau>0$ per use of the facility. ${ }^{1}$ Once the decision on the public good is taken, voter $i$ will choose the intensity of use $h_{i}$, which is non-negative and varies continuously. He or she maximizes a quasilinear benefit $b_{i}=w_{i}\left(h_{i}\right)+m_{i}-T_{i}$. In this expression, $m_{i}$ is voter $i$ 's income and $T_{i}$ is voter $i$ 's payment for the use of the facility. The function $w_{i}$ is strictly increasing and strictly concave up to some satiation point, where the marginal benefit $w_{i}^{\prime}$ is zero, and stays zero for higher intensities of use. Since some agents may have no preference at all for the public good, we allow the satiation point to be $h_{i}=0$.

If the public good is (is not) provided, we have $T_{i}=t\left(T_{i}=\tau h_{i}\right)$, and the resulting optimal use is denoted by $h_{i}^{1}\left(h_{i}^{0}\right)$. Since any additional use is costless once the public good is provided, $h_{i}^{1}$ is the satiation point, i.e., $w_{i}^{\prime}\left(h_{i}^{1}\right)=0$. The optimal value $h_{i}^{0}$ is given by the solution to the first order condition $w_{i}^{\prime}\left(h_{i}^{0}\right)=\tau$ if $w_{i}^{\prime}(0)>\tau$, and is $h_{i}^{0}=0$ if $w_{i}^{\prime}(0) \leq \tau$. Using these values in the benefit $b_{i}$, we find the indirect utility of individual $i$ in case the public good is provided, $b_{i}^{1}=w_{i}\left(h_{i}^{1}\right)+m_{i}-t$, and in case it is not provided, $b_{i}^{0}=w_{i}\left(h_{i}^{0}\right)+m_{i}-\tau h_{i}^{0}$.

We capture the other characteristics of the vote by a vector $\mathbf{a}_{i}$ for voter $i$. This vector has $K$ components labelled $k=1,2, \ldots, K$. For any vector $\mathbf{x}$, we define $\mathbf{x}_{(-k)}$ as the vector $\mathbf{x}$ without the component $k$. The vector $\mathbf{a}_{i}^{1}\left(\mathbf{a}_{i}^{0}\right)$ gives voter $i$ 's estimate of the situation with (without) the public good. Components $a_{k i}^{1}\left(a_{k i}^{0}\right)$,

\footnotetext{
${ }^{1}$ The price $\tau$ is to be interpreted as a payment for a normalized unit of use, say one visit to a local theater, one bus ride, or traveling a certain distance on local trains.
} 


\begin{tabular}{|c|c|c|c|}
\hline \multirow[b]{3}{*}{ votes } & \multirow[b]{3}{*}{$\begin{array}{l}\text { in favor } \\
\text { against }\end{array}$} & \multicolumn{2}{|c|}{ public good } \\
\hline & & is passed & is not passed \\
\hline & & $\begin{array}{c}\lambda_{i} b_{i}^{1}+\left(\boldsymbol{\zeta}_{i}+\boldsymbol{\psi}_{i}\right) \cdot \mathbf{a}_{i}^{1} \\
\lambda_{i} b_{i}^{1}+\boldsymbol{\zeta}_{i} \cdot \mathbf{a}_{i}^{1}+\boldsymbol{\psi}_{i} \cdot \mathbf{a}_{i}^{0}\end{array}$ & $\begin{array}{c}\lambda_{i} b_{i}^{0}+\boldsymbol{\zeta}_{i} \cdot \mathbf{a}_{i}^{0}+\boldsymbol{\psi}_{i} \cdot \mathbf{a}_{i}^{1} \\
\lambda_{i} b_{i}^{0}+\left(\boldsymbol{\zeta}_{i}+\boldsymbol{\psi}_{i}\right) \cdot \mathbf{a}_{i}^{0}\end{array}$ \\
\hline
\end{tabular}

Table 1: Utility of voter $i$ resulting from his or her vote and the outcome.

$k=1,2, \ldots, K$, describe, for example, voter $i$ 's estimate of other voters' monetary utility, the situation of local culture, environmental quality, or the supply and the quality of local transportation, if the public good is passed (is not passed). These aspects of the vote may give rise to social preferences or to expressive utility. In the first case, voter $i$ enjoys utility from the actual provision of the public good. For example, altruism in this sense means that voter $i$ casts his or her vote in order to change the outcome in the way others prefer. Voting based on social preferences is therefore necessarily instrumental, i.e., the voter wants to and expects to influence the decision with some probability larger than zero.

In contrast to this view, an influential strand of public choice literature (see Tullock, 1971; Hillman, 2010) has argued that voting tends to be expressive, at least in large electorates. In this case, utility is derived from the act of voting in a particular way. Then, the estimates $\mathbf{a}_{i}^{1}$ and $\mathbf{a}_{i}^{0}$ provide utility if the voter votes in favor of (against) the public good, irrespective of the actual outcome of the referendum. Expressive motivations could include voting which is like cheerleading (Brennan and Hamlin, 1998), a preference for expressing one's preference, or protest voting for the sake of protesting.

We assume that voter $i$ combines pocketbook and other considerations with linear preferences. Monetary benefits yield a marginal utility of $\lambda_{i}>0$, the weights of instrumental social concerns are given by the vector $\boldsymbol{\zeta}_{i}$, and the weights of expressive motives are given by the vector $\boldsymbol{\psi}_{i}$. Here, we assume that $\zeta_{k i} \psi_{k i} \geq 0$. This means that people do not have expressive benefits from voting against the outcome they find socially desirable. Moreover, we adopt the notational convention that $a_{k i}^{1}-a_{k i}^{0} \geq 0$ for all $k=1,2, \ldots, K$. This is without loss of generality since, if voter $i$ has a negative social or expressive valuation of component $k$, this is expressed by weights $\zeta_{k i} \leq 0$ and $\psi_{k i} \leq 0$. Furthermore, if a variable such as savings of others can take on positive and negative values, we can always split it in two non-negative variables. Depending on individual $i$ 's vote and the referendum outcome, four cases can arise, with utilities collected in Table $1 .^{2}$

Voter $i$ anticipates that by voting in favor of the public good rather than abstaining he or she increases the probability that the public good passes by $p_{i}^{1}$. Similarly,

\footnotetext{
${ }^{2}$ We discuss abstention in Section 3.2.
} 
by voting against the public good rather than abstaining he or she decreases the probability that the public good passes by $p_{i}^{0}$. Then, by switching his or her vote from no to yes he or she increases the probability that the public good passes by $p_{i}=p_{i}^{1}+p_{i}^{0}$. These subjective probabilities may but need not coincide with objective probabilities. We allow for the case where an individual considers his or her influence on the outcome to be negligible or zero, i.e. $p_{i}=0 .^{3}$

In line with Card et al. (2012), we derive $i$ 's preference on how to vote by comparing his or her expected utility if he or she votes in favor of or against the public good. From Table 1, the change in expected utility procured by switching from 'no' to 'yes' is $y_{i}=p_{i}\left[\lambda_{i}\left(b_{i}^{1}-b_{i}^{0}\right)+\boldsymbol{\zeta}_{i} \cdot\left(\mathbf{a}_{\mathbf{i}}^{\mathbf{1}}-\mathbf{a}_{i}^{0}\right)\right]+\boldsymbol{\psi}_{i} \cdot\left(\mathbf{a}_{i}^{1}-\mathbf{a}_{i}^{0}\right)$, or, equivalently,

$$
y_{i}=p_{i} \lambda_{i}\left(b_{i}^{1}-b_{i}^{0}\right)+\left(p_{i} \boldsymbol{\zeta}_{i}+\boldsymbol{\psi}_{i}\right) \cdot\left(\mathbf{a}_{i}^{1}-\mathbf{a}_{i}^{0}\right) .
$$

Individual $i$ votes for the public good if and only if $y_{i} \geq 0$.

In equation (1), the equivalent variation $b_{i}^{1}-b_{i}^{0}=w_{i}\left(h_{i}^{1}\right)-w_{i}\left(h_{i}^{0}\right)+\tau h_{i}^{0}-t$ is a monetary measure of the private gain individual $i$ obtains if the public good is provided, and the vector $\mathbf{a}_{i}^{1}-\mathbf{a}_{i}^{0}$ captures how the variables describing social and expressive motives change if the voter switches from 'no' to 'yes'.

The voting decision depends both on instrumental and expressive motivations:

Proposition 1. (i) For $p_{i}>0: y_{i} \gtreqless 0 \Longleftrightarrow b_{i}^{1}-b_{i}^{0} \gtreqless-\frac{\left(p_{i} \boldsymbol{\zeta}_{i}+\boldsymbol{\psi}_{i}\right) \cdot\left(\mathbf{a}_{i}^{1}-\mathbf{a}_{i}^{0}\right)}{p_{i} \lambda_{i}}$.

(ii) For $k=1,2, \ldots, K$ :

(a) if $p_{i} \zeta_{k i}+\psi_{k i} \gtrless 0$, then $y_{i} \gtrless 0 \Longleftrightarrow$

$$
a_{k i}^{1}-a_{k i}^{0}>-\frac{p_{i} \lambda_{i}\left(b_{i}^{1}-b_{i}^{0}\right)+\left(p_{i} \boldsymbol{\zeta}_{(-k) i}+\boldsymbol{\psi}_{(-k) i}\right) \cdot\left(\mathbf{a}_{(-k) i}^{1}-\mathbf{a}_{(-k) i}^{0}\right)}{p_{i} \zeta_{k i}+\psi_{k i}} ;
$$

(b) if $p_{i} \zeta_{k i}+\psi_{k i}=0$, then $\forall a_{k i}^{1}-a_{k i}^{0}$ :

$$
y_{i} \gtreqless 0 \Longleftrightarrow p_{i} \lambda_{i}\left(b_{i}^{1}-b_{i}^{0}\right)+\left(p_{i} \boldsymbol{\zeta}_{(-k) i}+\boldsymbol{\psi}_{(-k) i}\right) \cdot\left(\mathbf{a}_{(-k) i}^{1}-\mathbf{a}_{(-k) i}^{0}\right) \gtreqless 0 .
$$

\footnotetext{
${ }^{3}$ In our empirical application, the decision rule stipulates that the public good fails in case of a tie. Then, $p_{i}^{1}$ is the probability that among the voters other than $i$, there is an equal number of votes in favor and against the public good. Similarly, $p_{i}^{0}$ is the probability that among the other voters the number of favorable votes exceeds the number of negative votes by 1 . For an equilibrium analysis of the probability of affecting the voting outcome in a finite electorate, see Börgers (2004) and Taylor and Yildirim (2010).
} 
(iii) (a) If $\boldsymbol{\psi}_{i} \cdot\left(\mathbf{a}_{i}^{1}-\mathbf{a}_{i}^{0}\right) \gtrless 0$, then $y_{i} \gtrless 0 \Longleftrightarrow \lambda_{i}\left(b_{i}^{1}-b_{i}^{0}\right)+\boldsymbol{\zeta}_{i} \cdot\left(\mathbf{a}_{i}^{1}-\mathbf{a}_{i}^{0}\right) \underset{\leq}{\geq} 0$ or

$$
\begin{aligned}
& \left(\lambda_{i}\left(b_{i}^{1}-b_{i}^{0}\right)+\boldsymbol{\zeta}_{i} \cdot\left(\mathbf{a}_{i}^{1}-\mathbf{a}_{i}^{0}\right) \lessgtr 0 \text { and } p_{i}<-\frac{\boldsymbol{\psi}_{i} \cdot\left(\mathbf{a}_{i}^{1}-\mathbf{a}_{i}^{0}\right)}{\lambda_{i}\left(b_{i}^{1}-b_{i}^{0}\right)+\boldsymbol{\zeta}_{i} \cdot\left(\mathbf{a}_{i}^{1}-\mathbf{a}_{i}^{0}\right)}\right) ; \\
& \text { (b) if } p_{i}=0 \text {, then } y_{i} \gtreqless 0 \Longleftrightarrow \boldsymbol{\psi}_{i} \cdot\left(\mathbf{a}_{i}^{1}-\mathbf{a}_{i}^{0}\right) \gtreqless 0 .
\end{aligned}
$$

Proof. Each of the three claims follows from solving the relevant cases of $y_{i} \gtreqless 0$, using (1).

The first item in this proposition states that if the probability of being pivotal is strictly larger than zero, the individual will vote in line with own monetary interest provided this is sufficiently large. Point (a) in the second item states that if social or expressive preferences are present, their joint effect will determine the voting decision provided they are strong enough. For completeness, point (b) says that if social and expressive weights attached to some aspect are zero, the voting decision is independent of this aspect. The third item addresses the relative importance of expressive and instrumental motives. A voter who has expressive concerns which are strictly different from zero will always vote in line with these concerns when the probability of changing the outcome is sufficiently small. Then pocketbook benefits and instrumental social concerns should have no impact on the decision in an estimation based on equation (1).

The following corollary shows that for a voter without expressive concerns, the trade-off between pocketbook considerations and social preferences does not depend on the probability of changing the outcome:

Corollary 1. If $\boldsymbol{\psi}_{i} \cdot\left(\mathbf{a}_{i}^{1}-\mathbf{a}_{i}^{0}\right)=0$ and $p_{i}>0$, then

$$
y_{i} \gtreqless 0 \Longleftrightarrow b_{i}^{1}-b_{i}^{0} \gtreqless-\frac{\boldsymbol{\zeta}_{i} \cdot\left(\mathbf{a}_{i}^{1}-\mathbf{a}_{i}^{0}\right)}{\lambda_{i}} .
$$

Proof. The corollary follows directly from Proposition 1(i).

The corollary arises since all benefits from changing the outcome are weighted down equally. Notice that, while this argument does not literally hold if $p_{i}$ is exactly zero, by a trembling hand argument, a rational voter should still vote as if this probability were positive.

An econometric investigation of equation (1) will deliver estimates for the coefficient $\beta_{i}:=p_{i} \lambda_{i}$, which measures the impact of pocketbook motives, and the coefficients $\boldsymbol{\alpha}_{i}:=p_{i} \boldsymbol{\zeta}_{i}+\boldsymbol{\psi}_{i}$, which measure the joint impact of social and expressive concerns. It is worth noting that these coefficients are determined only up to a common scaling factor, as the sign of equation (1) is not affected if coefficients are 


$$
\begin{aligned}
& \begin{array}{ll}
\text { (a) } \boldsymbol{\psi}_{i}=0 . & \text { (b) } \boldsymbol{\psi}_{i} \neq 0 .
\end{array}
\end{aligned}
$$

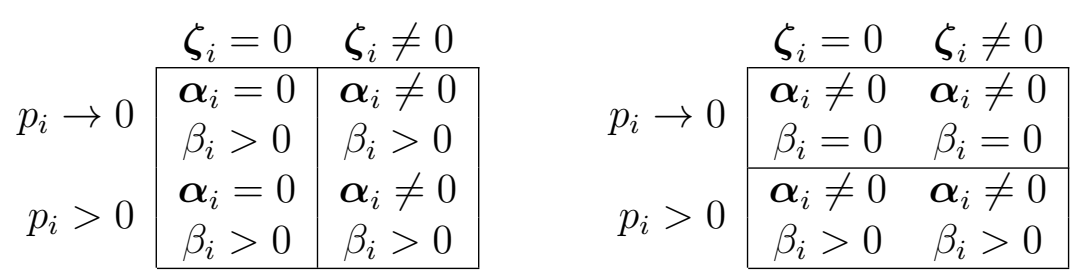

Table 2: Coefficients implied by the model when expressive motives are absent (a) and present (b).

multiplied by a positive constant. If voting depends on monetary benefits, we will estimate a positive coefficient $\beta_{i}>0$, provided the sample contains, among individuals with similar values of the variables $\mathbf{a}_{i}^{1}-\mathbf{a}_{i}^{0}$, both observations with $b_{i}^{1}-b_{i}^{0}$ small enough such that $y_{i}<0$ and large enough such that $y_{i} \geq 0$ (Proposition 1(i)). Similarly, if voting depends on $\mathbf{a}_{i}^{1}-\mathbf{a}_{i}^{0}$ and the sample contains, for similar values of monetary benefits, both observations with realizations of these variables such that $y_{i}<0$ and such that $y_{i} \geq 0$, we will estimate $\boldsymbol{\alpha}_{i} \neq 0$ (Proposition 1(ii)).

Table 2 summarizes the signs of the coefficients as implied by Proposition 1 and Corollary 1 . The table is organized according to alternative assumptions about the parameters of the model. The two panels (a) and (b) distinguish the cases where expressive motives are not or are present, ${ }^{4}$ and the columns distinguish the cases where social motives are not or are present. The rows relate to the voter's perception of his or her influence on the voting outcome. Here, $p_{i} \rightarrow 0\left(p_{i}>0\right)$ means that this influence is considered to be negligible (non-negligible).

Each cell gives the signs of the coefficients implied by the assumptions defining the cell. For example, in the upper-left cell of panel (a), neither social motives nor expressive concerns are present, so that one will find $\boldsymbol{\alpha}_{i}=0$. In addition, the voter does not think that he or she has a noticeable influence on the outcome. According to Corollary 1, however, his or her vote will still depend on monetary interests and hence the estimation will yield $\beta_{i}>0$. As is apparent from Table 2, different cells may yield the same prediction. This is highlighted by the lines in the two panels, which group constellations which lead to the same coefficients, that is, are observationally equivalent.

To test the implications of the model, we formulate two simple null-hypotheses about the coefficients:

H1 $\beta_{i}=0$,

\footnotetext{
${ }^{4}$ In Table 2, we associate the absence of expressive motives with the vector of weights being zero, $\boldsymbol{\psi}_{i}=0$. This is stronger than assuming $\boldsymbol{\psi}_{i} \cdot\left(\mathbf{a}_{i}^{1}-\mathbf{a}_{i}^{0}\right)=0$, since the latter may arise also if conflicting expressive concerns happen to cancel each other out.
} 
H2 $\alpha_{i}=0$.

The corresponding alternative hypotheses are $\beta_{i}>0$ for $\mathbf{H} \mathbf{1}$ and $\boldsymbol{\alpha}_{i} \neq 0$ for $\mathbf{H} \mathbf{2}$.

These hypotheses yield four possible cases. First, it may turn out that we cannot reject either of the two null-hypotheses. As is apparent from Table 2, this case cannot arise in the model, and hence, if this is the empirical outcome, the model must be considered as invalidated. This is the consequence of the assumption $\lambda_{i}>0$, which states that voters have a taste for money.

Second, we might reject Hypothesis $\mathbf{H 1}$ but not reject Hypothesis $\mathbf{H 2}$, as in the left half of Table 2 (a). This is the case of pure pocketbook voting. In this case, neither social nor expressive motives can be identified in the data.

Third, when Hypothesis $\mathbf{H} \mathbf{1}$ is not rejected and Hypothesis $\mathbf{H} \mathbf{2}$ is rejected, as in the upper half of Table 2 (b), we must conclude that voters consider the probability to affect the outcome to be negligible. This is the case of purely expressive voting. In this case, since voters believe that their influence on the outcome of the vote is negligible, social preferences also have a negligible impact. Thus, if voters care about benefits of others, this must be expressive, i.e., we can conclude that $\boldsymbol{\psi}_{i} \neq 0$. In this circumstance, we would have proved the existence of expressive motives.

Fourth, the empirical analysis may reject both null-hypotheses, lending support to the alternative hypotheses of non-zero coefficients for both kinds of variables. As can be seen in Table 2, this can arise in two circumstances. In the right half of panel (a), expressive motives are absent, but social motives alone produce a significant coefficient for the variables $\mathbf{a}_{i}^{1}-\mathbf{a}_{i}^{0}$. In the bottom half of panel (b), expressive motives are present and voters perceive the probability to affect the decision to be non-negligible. An empirical finding which rejects both hypotheses would be in contrast to the logic of purely expressive voting: The fact that pocketbook benefits are relevant in a large electorate indicates that either voters do not perceive their impact on the outcome to be negligible, or expressive motivations are negligible for the decision on how to vote.

In the fourth case, one has information only on the composite weight $\boldsymbol{\alpha}_{i}=$ $p_{i} \boldsymbol{\zeta}_{i}+\boldsymbol{\psi}_{i}$. One then cannot determine whether the aspects collected in the vector $\mathbf{a}_{i}$ are relevant because the individual cares about implemented policies, possibly including paternalism and other social preferences such as the environment, or because of expressive behavior relating to the same aspects. Hence, voting based on instrumental social preferences and voting based on expressive motives are observationally equivalent, as long as $p_{i}>0$.

To sum up, rejecting Hypothesis $\mathbf{H 1}$ establishes the relevance of pocketbook considerations for voting. Rejecting Hypothesis H2 shows that expressive motives, social preferences, or both affect the voting decision. A sufficient condition implying that this effect is due to expressive motives, however, would require that at the same 


\begin{tabular}{cr|cc|}
\multicolumn{2}{c}{ is passed } & public good \\
\cline { 3 - 4 } voter $i$ & participates & is not passed \\
\cline { 3 - 4 } & abstains & $\lambda_{i} b_{i}^{1}+\left(\boldsymbol{\zeta}_{i}+\boldsymbol{\psi}_{\boldsymbol{i}}\right) \cdot \mathbf{a}_{i}^{1}+d_{i}-c_{i}$ & $\lambda_{i} b_{i}^{0}+\boldsymbol{\zeta}_{i} \cdot \mathbf{a}_{i}^{0}+\boldsymbol{\psi}_{\boldsymbol{i}} \cdot \mathbf{a}_{i}^{1}+d_{i}-c_{i}$ \\
$\lambda_{i} b_{i}^{1}+\boldsymbol{\zeta}_{i} \cdot \mathbf{a}_{i}^{1}+\boldsymbol{\psi}_{\boldsymbol{i}} \cdot \mathbf{a}_{i}^{-}$ & $\lambda_{i} b_{i}^{0}+\boldsymbol{\zeta}_{i} \cdot \mathbf{a}_{i}^{0}+\boldsymbol{\psi}_{\boldsymbol{i}} \cdot \mathbf{a}_{i}^{-}$ \\
\cline { 3 - 5 } & & &
\end{tabular}

(a) $y_{i} \geq 0$.

public good

is passed is not passed

\begin{tabular}{rr|cc|}
\cline { 3 - 4 } voter $i$ & participates & $\lambda_{i} b_{i}^{1}+\boldsymbol{\zeta}_{i} \cdot \mathbf{a}_{i}^{1}+\boldsymbol{\psi}_{\boldsymbol{i}} \cdot \mathbf{a}_{i}^{0}+d_{i}-c_{i}$ & $\lambda_{i} b_{i}^{0}+\left(\boldsymbol{\zeta}_{i}+\boldsymbol{\psi}_{\boldsymbol{i}}\right) \cdot \mathbf{a}_{i}^{0}+d_{i}-c_{i}$ \\
& abstains & $\lambda_{i} b_{i}^{1}+\boldsymbol{\zeta}_{i} \cdot \mathbf{a}_{i}^{1}+\boldsymbol{\psi}_{\boldsymbol{i}} \cdot \mathbf{a}_{i}^{-}$ & $\lambda_{i} b_{i}^{0}+\boldsymbol{\zeta}_{i} \cdot \mathbf{a}_{i}^{0}+\boldsymbol{\psi}_{\boldsymbol{i}} \cdot \mathbf{a}_{i}^{-}$
\end{tabular}

(b) $y_{i}<0$.

Table 3: Utility of voter $i$ resulting from participating or abstaining. In panel a (b), the voter anticipates to vote in favor of (against) the public good.

time Hypothesis $\mathbf{H 1}$ is not rejected, i.e., one should not find an impact of monetary benefits on the voting decision.

\subsection{Participation decision}

We now turn to the decision whether to vote. Similar to the decision on how to vote, this decision depends on monetary benefits, social preferences, and expressive motivations. If the individual abstains, the variables describing expressive motives take on values $\mathbf{a}_{i}^{-}$, which in general may differ from the values $\mathbf{a}_{i}^{0}$ taken on if $i$ votes against and $\mathbf{a}_{i}^{1}$ taken on if $i$ votes in favor of the public good. In addition, as already observed by Riker and Ordeshook (1968), participation may induce costs and benefits unrelated to how the individual votes, which we denote by $c_{i}$ and $d_{i}$, respectively. Examples for $\operatorname{costs} c_{i}$ are the time needed to cast one's ballot or the effort to make up one's mind on how to vote. Examples for benefits $d_{i}$ are a civic duty motive for voting, the utility conferred by adhering to a social norm which advocates participation, or the benefit derived from not breaking with a long-formed habit of participating. We assume that the voter does not obtain the benefit $d_{i}$ if he or she casts an empty or spoilt ballot but that he or she still incurs the cost $c_{i}$ of voting. Therefore, casting an empty ballot is dominated by not turning out.

When calculating his or her utility, the voter anticipates that he or she will vote in favor of (against) providing the public good if $y_{i} \geq 0\left(y_{i}<0\right)$. The utilities resulting from the voter's choices in these two cases are collected in Tables 3(a) and (b). From Table 3(a), when $y_{i} \geq 0$, the difference between the expected utilities 
derived from participation and from abstention is given by

$$
z_{i}=p_{i}^{1}\left[\lambda_{i}\left(b_{i}^{1}-b_{i}^{0}\right)+\boldsymbol{\zeta}_{i} \cdot\left(\mathbf{a}_{i}^{1}-\mathbf{a}_{i}^{0}\right)\right]+\boldsymbol{\psi}_{i} \cdot\left(\mathbf{a}_{i}^{1}-\mathbf{a}_{i}^{-}\right)+d_{i}-c_{i} .
$$

Similarly, from Table 3(b), we find for $y_{i}<0$ :

$$
z_{i}=p_{i}^{0}\left[\lambda_{i}\left(b_{i}^{0}-b_{i}^{1}\right)+\boldsymbol{\zeta}_{i} \cdot\left(\mathbf{a}_{i}^{0}-\mathbf{a}_{i}^{1}\right)\right]+\boldsymbol{\psi}_{i} \cdot\left(\mathbf{a}_{i}^{0}-\mathbf{a}_{i}^{-}\right)+d_{i}-c_{i} .
$$

Using the indicators

$$
I_{i}^{1}=\left\{\begin{array}{ll}
1 & \text { if } y_{i} \geq 0 \\
0 & \text { otherwise }
\end{array} \quad \text { and } \quad I_{i}^{0}= \begin{cases}1 & \text { if } y_{i}<0 \\
0 & \text { otherwise }\end{cases}\right.
$$

for the expected vote, equations (2) and (3) can be presented as

$$
\begin{aligned}
z_{i}=\left(p_{i}^{1} I_{i}^{1}-p_{i}^{0} I_{i}^{0}\right)\left[\lambda_{i}\left(b_{i}^{1}-b_{i}^{0}\right)+\boldsymbol{\zeta}_{i} \cdot\left(\mathbf{a}_{i}^{1}-\mathbf{a}_{i}^{0}\right)\right] \\
+\boldsymbol{\psi}_{i} \cdot\left[I_{i}^{1}\left(\mathbf{a}_{i}^{1}-\mathbf{a}_{i}^{-}\right)+I_{i}^{0}\left(\mathbf{a}_{i}^{0}-\mathbf{a}_{i}^{-}\right)\right]+d_{i}-c_{i} .
\end{aligned}
$$

Individual $i$ will participate in the vote if $z_{i} \geq 0$.

Equation (4) takes a particularly simple form if one makes the additional assumption that expressive feelings created from abstention are the same as those created from voting against one's preferences, that is, $\mathbf{a}_{i}^{-}=\mathbf{a}_{i}^{0}$ if $y_{i} \geq 0$ and $\mathbf{a}_{i}^{-}=\mathbf{a}_{i}^{1}$ if $y_{i}<0$. Using this, equation (4) becomes

$$
\begin{aligned}
z_{i}=\left(p_{i}^{1} I_{i}^{1}-p_{i}^{0} I_{i}^{0}\right) \lambda_{i}\left(b_{i}^{1}-b_{i}^{0}\right) & \\
& +\left[\left(p_{i}^{1} \boldsymbol{\zeta}_{i}+\boldsymbol{\psi}_{i}\right) I_{i}^{1}-\left(p_{i}^{0} \boldsymbol{\zeta}_{i}+\boldsymbol{\psi}_{i}\right) I_{i}^{0}\right] \cdot\left(\mathbf{a}_{i}^{1}-\mathbf{a}_{i}^{0}\right)+d_{i}-c_{i} .
\end{aligned}
$$

Combining Proposition 1 and equation (5) shows how pocketbook considerations, social preferences, and expressive motives together with other benefits and costs of voting determine participation decisions:

Proposition 2. (i) (a) For $p_{i}^{1}>0, z_{i}>0$ and $y_{i}>0$ if and only if

$$
b_{i}^{1}-b_{i}^{0}>\max \left\{\frac{\left(p_{i} \boldsymbol{\zeta}_{i}+\boldsymbol{\psi}_{i}\right) \cdot\left(\mathbf{a}_{i}^{0}-\mathbf{a}_{i}^{1}\right)}{p_{i} \lambda_{i}}, \frac{\left(p_{i}^{1} \boldsymbol{\zeta}_{i}+\boldsymbol{\psi}_{i}\right) \cdot\left(\mathbf{a}_{i}^{0}-\mathbf{a}_{i}^{1}\right)}{p_{i}^{1} \lambda_{i}}+\frac{c_{i}-d_{i}}{p_{i}^{1} \lambda_{i}}\right\}
$$

(b) for $p_{i}^{0}>0, z_{i}>0$ and $y_{i}<0$ if and only if

$$
b_{i}^{0}-b_{i}^{1}>\max \left\{\frac{\left(p_{i} \boldsymbol{\zeta}_{i}+\boldsymbol{\psi}_{i}\right) \cdot\left(\mathbf{a}_{i}^{1}-\mathbf{a}_{i}^{0}\right)}{p_{i} \lambda_{i}}, \frac{\left(p_{i}^{0} \boldsymbol{\zeta}_{i}+\boldsymbol{\psi}_{i}\right) \cdot\left(\mathbf{a}_{i}^{1}-\mathbf{a}_{i}^{0}\right)}{p_{i}^{0} \lambda_{i}}+\frac{c_{i}-d_{i}}{p_{i}^{0} \lambda_{i}}\right\} .
$$

(ii) For $k=1,2, \ldots, K$ : 
(a) if $p_{i}^{1} \zeta_{k i}+\psi_{k i}>0$, then $z_{i}>0$ and $y_{i}>0$ if and only if

$$
\begin{aligned}
& a_{k i}^{1}-a_{k i}^{0}>\max \left\{\frac{p_{i} \lambda_{i}\left(b_{i}^{0}-b_{i}^{1}\right)+\left(p_{i} \boldsymbol{\zeta}_{(-k) i}+\boldsymbol{\psi}_{(-k) i}\right) \cdot\left(\mathbf{a}_{(-k) i}^{0}-\mathbf{a}_{(-k) i}^{1}\right)}{p_{i} \zeta_{k i}+\psi_{k i}},\right. \\
& \left.\quad \frac{p_{i}^{1} \lambda_{i}\left(b_{i}^{0}-b_{i}^{1}\right)+\left(p_{i}^{1} \boldsymbol{\zeta}_{(-k) i}+\boldsymbol{\psi}_{(-k) i}\right) \cdot\left(\mathbf{a}_{(-k) i}^{0}-\mathbf{a}_{(-k) i}^{1}\right)}{p_{i}^{1} \zeta_{k i}+\psi_{k i}}+\frac{c_{i}-d_{i}}{p_{i}^{1} \zeta_{k i}+\psi_{k i}}\right\} ;
\end{aligned}
$$

(b) if $p_{i}^{0} \zeta_{k i}+\psi_{k i}<0$, then $z_{i}>0$ and $y_{i}<0$ if and only if

$$
\begin{aligned}
& a_{k i}^{1}-a_{k i}^{0}>\max \left\{\frac{p_{i} \lambda_{i}\left(b_{i}^{1}-b_{i}^{0}\right)+\left(p_{i} \boldsymbol{\zeta}_{(-k) i}+\boldsymbol{\psi}_{(-k) i}\right) \cdot\left(\mathbf{a}_{(-k) i}^{1}-\mathbf{a}_{(-k) i}^{0}\right)}{-\left(p_{i} \zeta_{k i}+\psi_{k i}\right)},\right. \\
& \left.\quad \frac{p_{i}^{0} \lambda_{i}\left(b_{i}^{1}-b_{i}^{0}\right)+\left(p_{i}^{0} \boldsymbol{\zeta}_{(-k) i}+\boldsymbol{\psi}_{(-k) i}\right) \cdot\left(\mathbf{a}_{(-k) i}^{1}-\mathbf{a}_{(-k) i}^{0}\right)}{-\left(p_{i}^{0} \zeta_{k i}+\psi_{k i}\right)}+\frac{c_{i}-d_{i}}{-\left(p_{i}^{0} \zeta_{k i}+\psi_{k i}\right)}\right\} .
\end{aligned}
$$

Proof: Claim (i)(a). Observe first that $p_{i}^{1}>0$ implies $p_{i}>0$. Thus, by Proposition $1(\mathrm{i}), b_{i}^{1}-b_{i}^{0}>\left(p_{i} \boldsymbol{\zeta}_{i}+\boldsymbol{\psi}_{i}\right) \cdot\left(\mathbf{a}_{i}^{0}-\mathbf{a}_{i}^{1}\right) /\left(p_{i} \lambda_{i}\right)$ is equivalent to $y_{i}>0$. Hence, $I_{i}^{1}=1$ and $I_{i}^{0}=0$. Inserting these indicators in equation (5) and solving $z_{i}>0$ yields $b_{i}^{1}-b_{i}^{0}>\left(p_{i}^{1} \boldsymbol{\zeta}_{i}+\boldsymbol{\psi}_{i}\right) \cdot\left(\mathbf{a}_{i}^{0}-\mathbf{a}_{i}^{1}\right) /\left(p_{i}^{1} \lambda_{i}\right)+\left(c_{i}-d_{i}\right) /\left(p_{i}^{1} \lambda_{i}\right)$. The two conditions given in the claim are jointly satisfied if and only if $y_{i}>0$ and $z_{i}>0$. The other claims follow from the same logic.

The first part of this proposition states that sufficiently large monetary gains guarantee participation and voting in favor of the public good, and sufficiently large monetary losses guarantee participation and voting against. The second part states that if some social or expressive concern is sufficiently strong, the individual will participate and vote in line with his or her evaluation of this concern.

If $c_{i}-d_{i}>0$, implying a net cost of voting, participation requires either a sufficiently strong expressive motivation, or sufficiently different valuations of outcomes combined with a sufficiently large subjective probability of affecting the outcome. In particular, if expressive concerns are absent, then even substantial monetary stakes do not guarantee participation if that probability is negligible. This contrasts with the voting decision where, as shown in Corollary 1, the probability of changing the outcome is not relevant when the individual has no expressive preferences. Consequently, the overestimation of small probabilities which is often found in experiments $^{5}$ can be expected to play a bigger role in the turnout than in the voting

\footnotetext{
${ }^{5}$ Already Kahneman and Tversky (1979) mention that people often overestimate probabilities of rare events. For experimental evidence on overestimation of the probability to affect an election outcome, see Duffy and Tavits (2008).
} 
decision.

If one finds that pocketbook motives play a role in determining participation, then this suggests that the subjective probability of changing the outcome is not negligible. Consequently, in this case, it is not possible to disentangle instrumental social preferences and expressive motives. Similar to the voting decision, these two motivations are observationally equivalent if pocketbook considerations contribute to explaining participation. If, instead, pocketbook considerations are not found to affect the participation decision, then it is plausible that the joint effect of instrumental social concerns and expressive motives is driven by the latter.

Even for $c_{i}-d_{i}=0$, the individual may abstain. Proposition 2(i) implies:

Corollary 2. If $c_{i}-d_{i}=0$, then $z_{i}<0$ if

(a) $\boldsymbol{\psi}_{i} \cdot\left(\mathbf{a}_{i}^{0}-\mathbf{a}_{i}^{1}\right)>0$ and

$$
\left(\frac{\boldsymbol{\zeta}_{i}}{\lambda_{i}}+\frac{\boldsymbol{\psi}_{i}}{p_{i} \lambda_{i}}\right) \cdot\left(\mathbf{a}_{i}^{0}-\mathbf{a}_{i}^{1}\right)<b_{i}^{1}-b_{i}^{0}<\left(\frac{\boldsymbol{\zeta}_{i}}{\lambda_{i}}+\frac{\boldsymbol{\psi}_{i}}{p_{i}^{1} \lambda_{i}}\right) \cdot\left(\mathbf{a}_{i}^{0}-\mathbf{a}_{i}^{1}\right) ; \quad \text { or }
$$

(b) $\boldsymbol{\psi}_{i} \cdot\left(\mathbf{a}_{i}^{1}-\mathbf{a}_{i}^{0}\right)>0$ and

$$
\left(\frac{\boldsymbol{\zeta}_{i}}{\lambda_{i}}+\frac{\boldsymbol{\psi}_{i}}{p_{i} \lambda_{i}}\right) \cdot\left(\mathbf{a}_{i}^{1}-\mathbf{a}_{i}^{0}\right)<b_{i}^{0}-b_{i}^{1}<\left(\frac{\boldsymbol{\zeta}_{i}}{\lambda_{i}}+\frac{\boldsymbol{\psi}_{i}}{p_{i}^{0} \lambda_{i}}\right) \cdot\left(\mathbf{a}_{i}^{1}-\mathbf{a}_{i}^{0}\right) .
$$

If pocketbook and expressive motivations go in opposite directions the individual will vote in line with expressive concerns if potential private gains or losses are small, and in line with pocketbook concerns if private gains or losses are large. However, there is an intermediate range, as given in the corollary, where rational abstention may occur. This is because casting a different vote affects the probability of the outcome which is better from a pocketbook perspective by more than abstaining, i.e. $p_{i}>p_{i}^{0}, p_{i}^{1}$. Moreover, we assume that expressive feelings from abstention are the same as expressive feelings from voting differently than one would have actually done. Therefore, in a situation where there is a conflict between pocketbook and expressive concerns, abstaining is a cheaper way of obtaining the expressive benefit than voting against one's pocketbook interest.

\section{Data}

\subsection{Institutional background}

University of Goettingen holds referenda among students on whether they should collectively purchase flat rate tickets, called 'Semestertickets'. These tickets give 
all students at the university the right to use a specific service as often as they wish. The price of a ticket is very attractive compared with prices for individual use; however, once a ticket is accepted in the referendum, its price is collected as part of the registration fee from all students with very few exemptions.

Referenda are usually held yearly over at least three consecutive days in January at several locations on campus, and voting by mail is possible. For a ticket to be accepted, a double threshold must be passed: more than $50 \%$ of the votes must be in favor of the ticket, and, at the same time, at least $15 \%$ of the total number of students must vote in favor.

We analyze votes on tickets for regional trains, local buses, and cultural amenities. The most important ticket is the train ticket which grants free travel on local trains. It was introduced in 2004. Until 2010, it covered, with only minor changes, all of the tracks depicted in Figure 1, served by several operators. The vote in 2010, however, was preceded by complaints from student representatives about the price charged by Deutsche Bahn for its section of the tracks. As a result, the ticket was split in two. The first ticket covered the offer by the two companies Metronom and Cantus, henceforth called the MetroCan ticket (the tracks are depicted as solid red lines in Figure 1), and was approved in January 2010. The second ticket covered the tracks served by Deutsche Bahn and two smaller companies (depicted as blue dashed lines in Figure 1). The latter companies are jointly referred to as Bahn throughout this paper. After some negotiations with Bahn, a referendum on the Bahn ticket was held in May 2010. The ticket cost 42.24 euros per semester. Of approximately 22,800 students registered at that time, $25 \%$ participated in the referendum, of whom $77 \%$ voted yes.

In subsequent years the train ticket proposal again covered all tracks. The culture ticket was introduced in October 2012. It offers free or highly discounted entrance to a number of cultural institutions and events, such as theaters, museums, and concerts. The bus ticket, which covers all buses within Goettingen and two nearby villages, would have been a novelty in 2013. In the 2013 referenda, the prices per semester amounted to 8.55 euros for the culture ticket, 25.80 euros for the bus ticket and 95.04 euros for the train ticket, and approximately $36 \%$ of almost 25,600 students took part in each referendum. Two of the three referenda were close. While the culture ticket just passed with $53 \%$ approval, the bus ticket failed with $46 \%$ support. An overwhelming majority of $82 \%$ voted in favor of the train ticket.

\subsection{Dataset I}

Information in Dataset I refers to the referendum on the train ticket covering the Bahn tracks in May 2010. To obtain data on the voting and traveling behavior 
Figure 1: Map

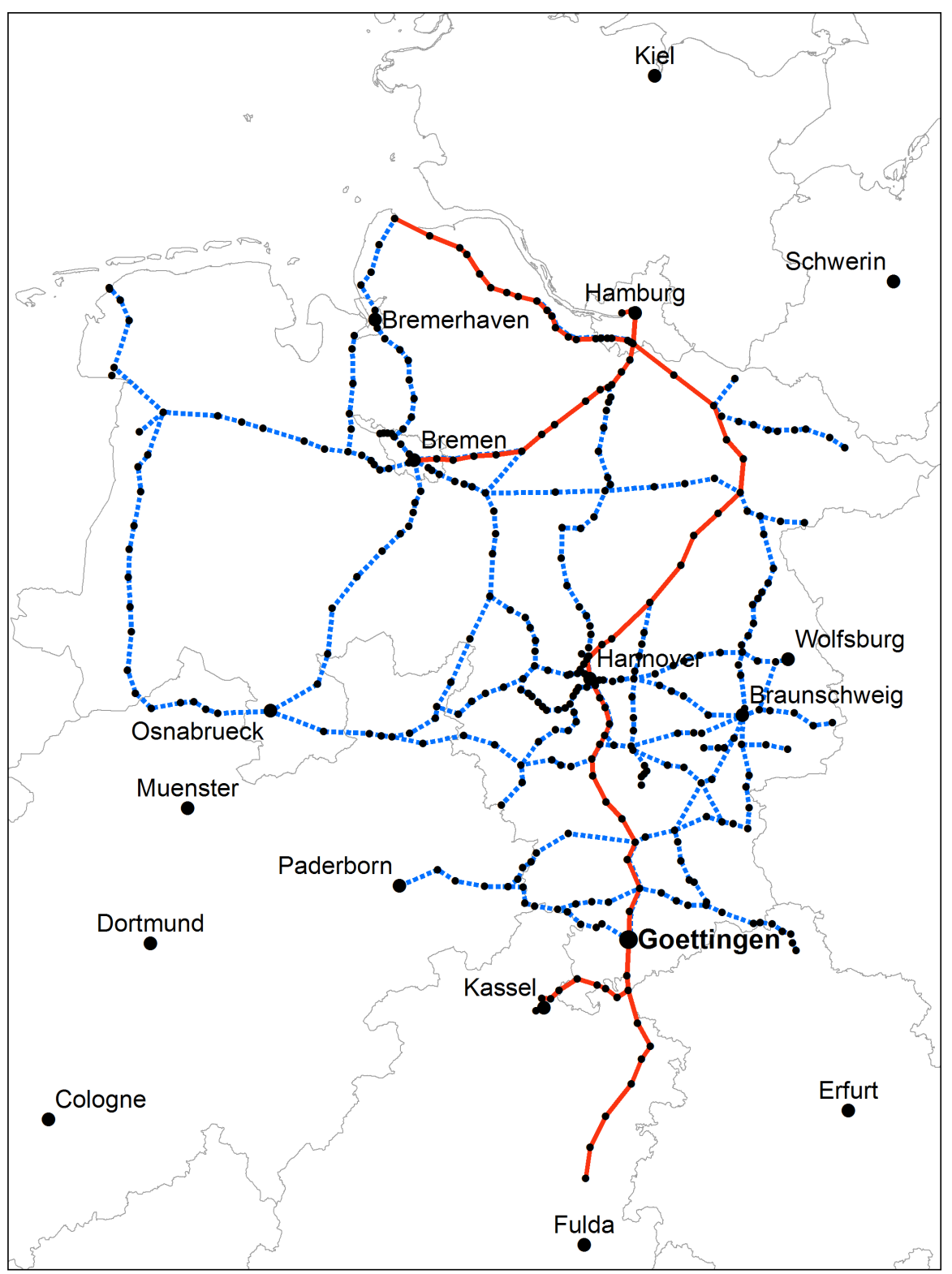

The tracks covered by the Bahn (dashed blue lines) and MetroCan (solid red lines) tickets. The gray lines are state boundaries. 
Table 4: Summary statistics Dataset I

\begin{tabular}{|c|c|c|c|c|}
\hline \multirow[b]{2}{*}{ Variable } & \multicolumn{2}{|c|}{ All } & \multicolumn{2}{|c|}{ Vote on Bahn ticket $=1$} \\
\hline & $\mathrm{N}$ & Mean & $\mathrm{N}$ & Mean \\
\hline Vote on Bahn ticket & 1189 & 0.70 & 828 & 1.00 \\
\hline Bahn ticket: yes & 818 & 0.68 & 818 & 0.68 \\
\hline Savings $\diamond$ & 1189 & 255.09 & 828 & 302.92 \\
\hline Own price threshold ${ }^{\mathbf{s}}$ & 1125 & 69.76 & 783 & 72.31 \\
\hline Exp. ave. price threshold ${ }^{\curvearrowright}$ & 1099 & 63.20 & 764 & 63.03 \\
\hline Leisure/work & 1189 & 0.06 & 828 & 0.06 \\
\hline Visiting others & 1174 & 0.65 & 819 & 0.67 \\
\hline Female & 1176 & 0.57 & 817 & 0.54 \\
\hline Freshman & 1099 & 0.15 & 768 & 0.18 \\
\hline Altruist $(-)$ & 1074 & 0.14 & 741 & 0.13 \\
\hline Altruist $(+)$ & 1074 & 0.34 & 741 & 0.33 \\
\hline Protest & 1189 & 0.21 & 828 & 0.24 \\
\hline Christian Democrats & 911 & 0.21 & 645 & 0.22 \\
\hline Social Democrats & 911 & 0.24 & 645 & 0.27 \\
\hline Liberal Democrats & 911 & 0.11 & 645 & 0.11 \\
\hline Green & 911 & 0.35 & 645 & 0.33 \\
\hline Left & 911 & 0.03 & 645 & 0.02 \\
\hline Other parties & 911 & 0.05 & 645 & 0.05 \\
\hline
\end{tabular}

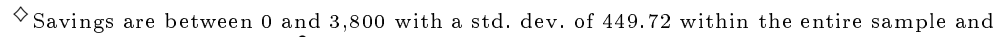
487.99 among the voters; *own price thresholds are between 0 and 750 with a std. dev. of 65.22 and 64.14 , respectively; ${ }^{\circ}$ and expected average price thresholds are between 10 and 720 with a std. dev. of 47.92 and 46.76 , respectively.

of students, an anonymous online survey was conducted. Unlike exit polls, this method allows non-voters to be included in the dataset. To incentivize participation, students were invited to take part in a lottery with prizes including 250 euros and 15 pairs of tickets to a local cinema. The survey was open from July 6 until November 11, 2010.

Summary statistics for Dataset I are reported in Table 4. This dataset consists of 1,189 observations after cleaning the data. ${ }^{6}$ Of these, 828 students took part in the referendum, showing an overrepresentation of voters in our sample. At the same time, these data allow us to base our analysis on detailed information on almost one sixth of all voters in the referendum. Among the voters in the sample, the share of yes votes is $68 \%$ and hence smaller than the share of yes votes in the polling box.

The key variable in this dataset which allows us to measure pocketbook benefits is the individual savings of each student. We construct an objective measure of the savings associated with the Bahn ticket by combining the number of trips to visit the respondent's parents using this ticket within the previous 12 months with the price that would have been paid in the absence of the ticket. ${ }^{7}$ As seen in the note

\footnotetext{
${ }^{6}$ See Appendix A.I in the supplementary material for a detailed description.
}

${ }^{7} \mathrm{~A}$ detailed description of the calculation of savings is included as Appendix A.II in the supple- 
below Table 4, the savings variable exhibits a large variation. We focus on trips to visit parents because this is the most common trip students make. Moreover, the two larger cities close to Goettingen, namely Hannover and Kassel, which might be attractive leisure destinations, can be reached using the MetroCan ticket (Figure 1). We define the difference between savings and the ticket price as net savings. In the econometric analysis, we use a transformation of net savings to measure pocketbook benefits $b_{i}^{1}-b_{i}^{0}$, as explained in Section 6.1.

A limitation of the savings variable is that students can also use the ticket for other trips than visiting their parents. To complement the savings measure, we asked whether the respondent visited people other than his or her parents using the ticket. We coded this information as a binary variable since quantifying monetary savings from these trips would have required the zip codes of other people visited and the number of times each of them was visited.

The key variables measuring social preferences or expressive concerns describe whether a student considered benefits of others in his or her voting decision and his or her evaluation of these benefits. These variables are based upon the answers to three questions. The first asks what is the highest price at which the respondent would vote in favor of the Bahn ticket. The second asks about the respondent's belief about the corresponding average of fellow students. The third question asks how the respondent weighted these two considerations in his or her vote. If the voting decision is influenced by the belief about the average preferences of other students, then the respondent is classified as an altruist. The resulting group of altruists is then split into those who think that students on average will gain from this ticket and those who think that students on average will lose. Accordingly, altruist $(+)$ is equal to one if the student is an altruist and believes that the price threshold of fellow students is on average at least as large as the price, and zero otherwise. We define altruist(-) analogously. If the student did not vote, then the third question on the actual voting decision is replaced by a corresponding question about a hypothetical voting decision. In the econometric analysis, the binary variables altruist $(+)$ and altruist(-) are components of the vector $\left(\mathbf{a}_{i}^{1}-\mathbf{a}_{i}^{0}\right)$ representing social preferences or expressive concerns.

The questionnaire also allowed students to enter free text regarding the primary reasons to vote for or against the Bahn ticket. To use this qualitative information, a content analysis was performed to identify the relevant topics. Afterward, three raters independently coded all of the answers with respect to whether a topic did appear. Finally, an indicator variable was defined that is equal to one if at least two of the three raters independently identified the topic in the statement given and zero otherwise. We use two variables resulting from this qualitative analysis. The first

mentary material. 
item, leisure/work, complements our measures of pocketbook benefits. It captures whether the student mentioned leisure activities other than visiting people, such as exploring the region, or work-related usage. The second item emerging from the content analysis is protest: some students expressed their unwillingness to accept the price of the ticket or feared that accepting the conditions would foster future price increases. It is plausible that this variable represents primarily expressive concerns, but it can also be viewed as a social consideration if students strategically cast a protest vote. Among the voters, the shares of students referring to leisure/work and protest are approximately $6 \%$ and $24 \%$, respectively.

The control variables in this dataset include gender, the party for which the student voted in the federal election in 2009 and whether the student is a freshman. Being a freshman is relevant because the first-year students in the dataset only began university in October 2009. Thus, they could not use the ticket for a full year which biases the savings variable downwards.

\subsection{Dataset II}

Dataset II covers the referenda on all three tickets that took place in January 2013 and was collected using exit polls. After leaving the polling place, students were approached by members of the survey team and asked to take part in a paper-based survey. To preserve anonymity, cubicles similar to polling booths were installed. Participation was incentivized by a lottery with prizes of 200, 100 and 50 euros.

After excluding those students who did not provide any voting decision, Dataset II contains 1,334 observations. Summary statistics are shown in Table 5. Within our sample, the shares of yes votes for all three tickets are slightly higher than the respective overall shares. The students in the dataset would have just passed the bus ticket, which narrowly missed the $50 \%$ approval threshold in the referendum. However, because our sample contains detailed information on one seventh of all the votes cast, we are confident that these deviations are of minor importance when analyzing individual voting decisions.

Pocketbook benefits $b_{i}^{1}-b_{i}^{0}$ are measured by categorical variables on the intensity of use, defined differently for the tickets (Table 5). For the train and culture tickets, students were asked about their use of these amenities within the last 12 months and the year before the ticket had been introduced, respectively. For the bus ticket, the intensity of use refers to teaching periods during term. In addition, students were asked if they would buy the ticket for themselves if the ticket were rejected in the referendum but were available for purchase at the same price on an individual basis. This provides a revealed preference measure of to what extent pocketbook benefits alone rationalize voting decisions on each ticket. 
Table 5: Summary statistics Dataset II

\begin{tabular}{|c|c|c|}
\hline Variable & $\mathrm{N}$ & Mean \\
\hline \multicolumn{3}{|l|}{ Train ticket } \\
\hline Train ticket: yes & 1252 & 0.86 \\
\hline Would buy it & 1248 & 0.64 \\
\hline Never & 1321 & 0.07 \\
\hline Rarely ( $\leq 5 /$ year $)$ & 1321 & 0.17 \\
\hline Sometimes (monthly) & 1321 & 0.32 \\
\hline Often (weekly) & 1321 & 0.21 \\
\hline Very often ( $\geq 2$ /week $)$ & 1321 & 0.24 \\
\hline Savings to others important & 1292 & 0.47 \\
\hline Environment important & 1284 & 0.38 \\
\hline \multicolumn{3}{|l|}{ Bus ticket $\diamond$} \\
\hline Bus ticket: yes & 1246 & 0.51 \\
\hline Would buy it & 1276 & 0.37 \\
\hline Never & 1329 & 0.40 \\
\hline Rarely ( 1 or 2 /semester) & 1329 & 0.24 \\
\hline Sometimes (monthly) & 1329 & 0.13 \\
\hline Often (weekly) & 1329 & 0.09 \\
\hline Very often $(\geq 2$ /week $)$ & 1329 & 0.14 \\
\hline Savings to others important & 1280 & 0.23 \\
\hline Strengthening bus important & 1245 & 0.12 \\
\hline \multicolumn{3}{|l|}{ Culture ticket } \\
\hline Culture ticket: yes & 1283 & 0.54 \\
\hline Would buy it & 1233 & 0.44 \\
\hline Never & 1234 & 0.56 \\
\hline Rarely ( 1 or $2 /$ year) & 1234 & 0.25 \\
\hline Sometimes ( 3 to $5 /$ year) & 1234 & 0.12 \\
\hline Often (6 to $10 /$ year) & 1234 & 0.04 \\
\hline Very often $(>10 /$ year $)$ & 1234 & 0.03 \\
\hline Savings to others important & 1235 & 0.24 \\
\hline Others should go important & 1201 & 0.29 \\
\hline Strengthening local culture important & 1229 & 0.39 \\
\hline \multicolumn{3}{|l|}{ Control variables } \\
\hline Female & 1276 & 0.50 \\
\hline Freshman & 1318 & 0.30 \\
\hline Christian Democrats & 1140 & 0.26 \\
\hline Social Democrats & 1140 & 0.29 \\
\hline Liberal Democrats & 1140 & 0.04 \\
\hline Green & 1140 & 0.31 \\
\hline Left & 1140 & 0.05 \\
\hline Other parties & 1140 & 0.05 \\
\hline Economic sciences & 1322 & 0.30 \\
\hline Social sciences & 1322 & 0.24 \\
\hline Forestry/Agriculture & 1322 & 0.06 \\
\hline Humanities & 1322 & 0.27 \\
\hline Geology/Geography & 1322 & 0.03 \\
\hline Law & 1322 & 0.11 \\
\hline Natural sciences & 1322 & 0.08 \\
\hline Other fields & 1322 & 0.03 \\
\hline
\end{tabular}

$\diamond$ Intensity of the use of the bus ticket refers to the lecture period. 
For each of the three tickets, the vector $\left(\mathbf{a}_{i}^{1}-\mathbf{a}_{i}^{0}\right)$ representing social preferences or expressive concerns contains as a common component information on whether the respondent considered savings to other students to be important in his or her vote. The answers to these questions were given on a four-point Likert scale ranging from 'not important' to 'important'. Furthermore, we asked about other motives, such as environmental aspects in the case of the train ticket or strengthening local transportation or local cultural life in the case of the bus or culture tickets, respectively, using the same Likert scale. In Table 5, we give the shares of students who replied that these other considerations were important. This binary coding is also used in the regression analysis presented in Section 6.

Additional control variables include gender, fields of study, ${ }^{8}$ and being a freshman. Moreover, political preferences were captured by a question on how the respondent would vote in a federal election if this election were to take place the following Sunday.

\section{The big picture}

In this section, we take a closer look at the data in a descriptive analysis.

\subsection{The voting decision}

The big picture that emerges is that there is strong evidence for pocketbook voting but that social preferences or expressive motivations also play an important role. In terms of the theory, this means that both null-hypotheses $\mathbf{H 1}$ and $\mathbf{H} \mathbf{2}$ are refuted. As discussed in Section 3.1, this suggests that either voters expect to have a non-negligible impact on the outcome, or that expressive voting is weak for a vast majority of voters. Hence, our findings rule out the case of purely expressive voting.

We first give the evidence for pocketbook voting in the 2010 vote on the Bahn ticket. For this purpose, we combine the savings variable with the information on whether students used the ticket for visits other than those to their parents. This allows us to define those for whom savings from visiting parents were less than the price of the ticket and who neither visit other people using the ticket nor mention leisure or work-related trips as losers in terms of private benefits. Similarly, we can classify those for whom the savings from visiting parents exceeded the price of the ticket as winners. Those for whom savings from visiting parents fell short of the price of the ticket but who also mentioned other trips are a middle category, in

\footnotetext{
${ }^{8}$ Due to the high number of polling stations, the survey team could not cover all of the stations during open hours on all three days. Therefore, the faculties of Law, Humanities, Economic Sciences and Social Sciences are overrepresented in the dataset.
} 
which we cannot say for sure whether the student in question privately gained or lost from the ticket. Of the 815 voters in Dataset I, $24 \%$ are classified as losers and $49 \%$ as winners.

Figure 2 depicts the share of yes votes for losers, the middle category, and winners. To illustrate how voting depends on the magnitude of pocketbook gains, the share of yes votes is calculated separately for each quartile of savings among the winners. The picture that we find is very much in line with pocketbook voting. Overall, $92 \%$ of the winners voted in favor of the ticket and $75 \%$ of the losers against. Among the winners, we see that the share of yes votes increases from $80 \%$ in the first quartile to $98 \%$ in the last.

Figure 2: Savings and share of yes votes - Dataset I

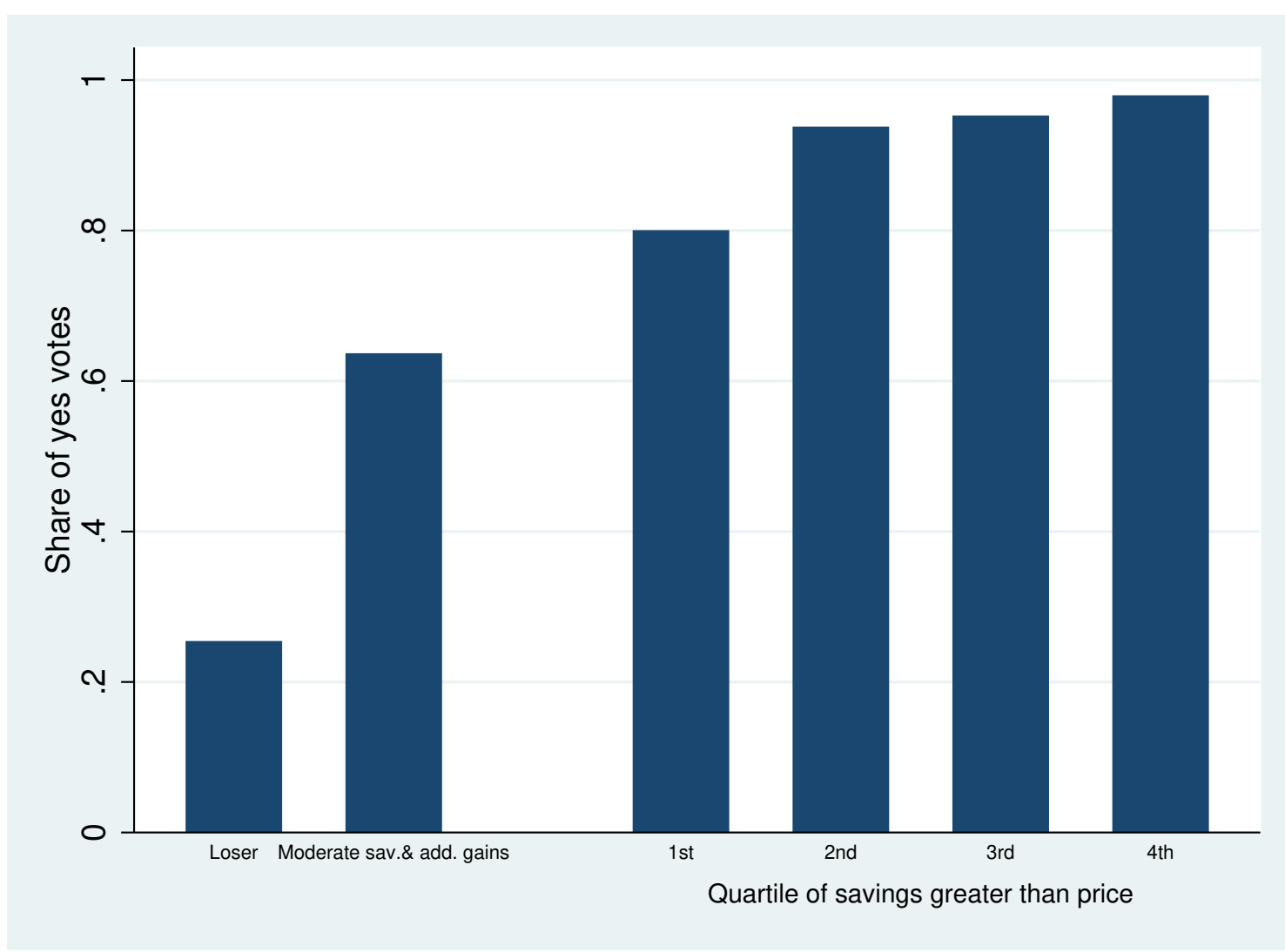

Losers' savings do not cover the ticket price, and they neither visit other people using the ticket nor mention leisure/work usage. The savings of the second group alone do not cover the ticket price; however, they mention other trips. The last four bars refer to respondents whose savings exceed the ticket price.

A corresponding picture of strong pocketbook voting also arises from Dataset II. Figure 3 depicts the share of yes votes depending on how intensively the voter 
used the service that was the subject of the vote. For each ticket, more than $90 \%$ of those who used the service very often voted in favor, while the share of yes votes varies between $24 \%$ and $32 \%$ for those who never used the service.

Figure 3: Intensity of use and yes votes - Dataset II

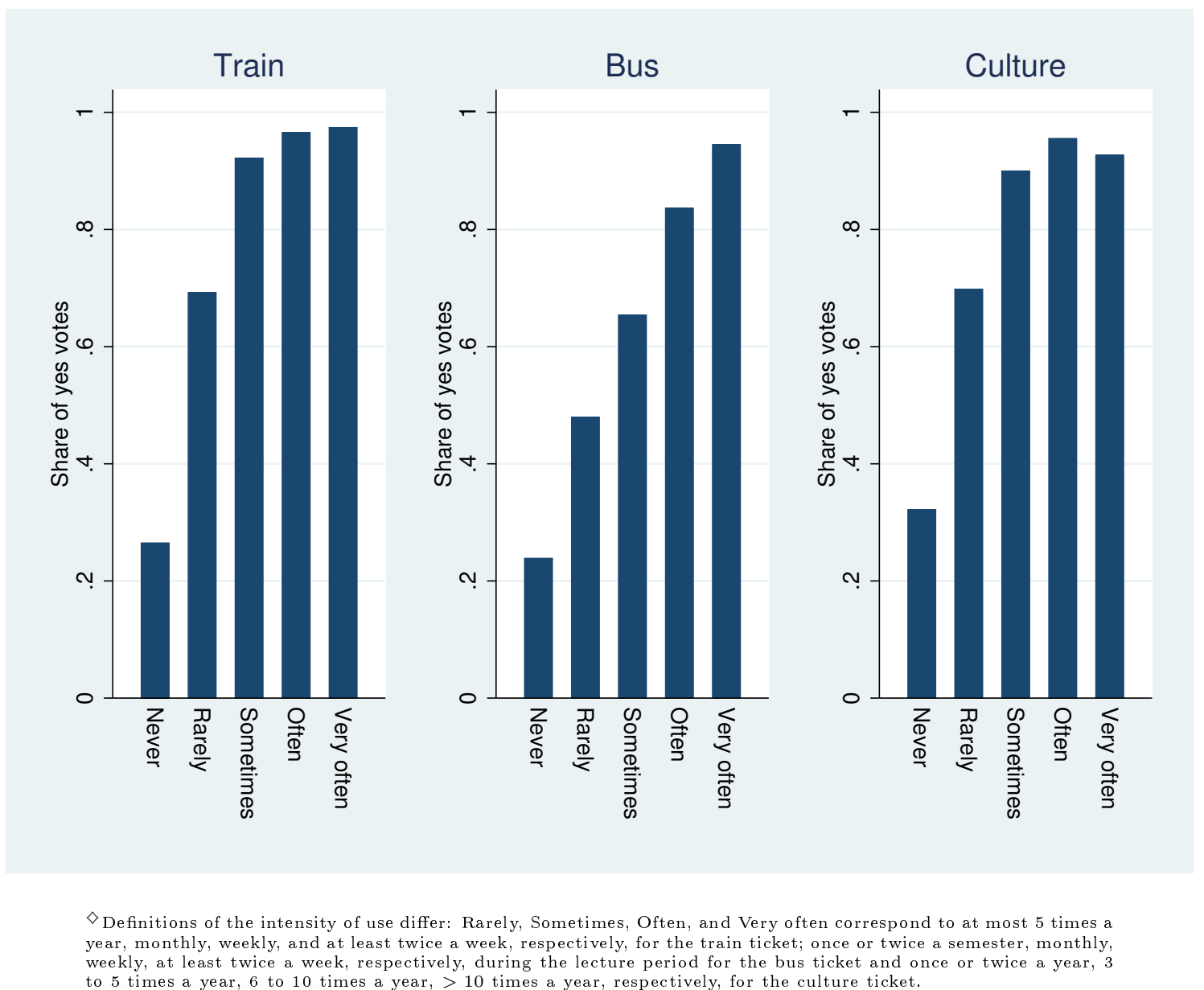

We complement the analysis of to what extent objective measures of use can explain voting with a comparison between actual voting and hypothetical choices as private consumers. In Dataset II, we asked respondents whether they would have bought the ticket individually if it were rejected in the referendum but available for individual purchase at the same price. If voting exclusively followed pocketbook considerations, we would expect those who voted in favor to also be willing to buy the ticket and those who voted against to be unwilling to do so. Table 6 shows that $93 \%$ to $96 \%$ of those who voted against a semester ticket would also decline 
Table 6: Pocketbook voting - Dataset II

\begin{tabular}{c|cc|c}
\hline \hline \multicolumn{4}{|c}{ Train ticket } \\
\hline \multirow{2}{*}{ Buy it } & \multicolumn{2}{|c}{ Vote } & \multirow{2}{*}{ Total } \\
& No & Yes & \\
\hline No & 158 & 256 & 414 \\
Yes & 11 & 755 & 766 \\
\hline Total & 169 & 1,011 & 1,180 \\
\hline \hline
\end{tabular}

\begin{tabular}{c|cc|c}
\hline \hline \multicolumn{4}{|c}{ Bus ticket } \\
\hline \multirow{2}{*}{ Buy it } & \multicolumn{2}{|c}{ Vote } & \multirow{2}{*}{ Total } \\
& No & Yes & \\
\hline No & 572 & 160 & 732 \\
Yes & 21 & 441 & 462 \\
\hline Total & 593 & 601 & 1,194 \\
\hline \hline
\end{tabular}

\begin{tabular}{c|cc|c}
\hline \hline \multicolumn{4}{c}{ Culture ticket } \\
\hline \multirow{2}{*}{ Buy it } & \multicolumn{2}{|c}{ Vote } & \multirow{2}{*}{ Total } \\
& No & Yes & \\
\hline No & 519 & 147 & 666 \\
Yes & 27 & 495 & 522 \\
\hline Total & 546 & 642 & 1,188 \\
\hline \hline
\end{tabular}

Table 7: Social preferences and protest among winners and losers - Dataset I

\begin{tabular}{|c|c|c|c|}
\hline \multicolumn{4}{|c|}{ Bahn ticket, only losers } \\
\hline \multirow{2}{*}{ Altruist $(+)$} & \multicolumn{2}{|c|}{ Vote } & \multirow{2}{*}{ Total } \\
\hline & No & Yes & \\
\hline No & 119 & 17 & 136 \\
\hline Yes & 12 & 23 & 35 \\
\hline Total & 131 & 40 & 171 \\
\hline
\end{tabular}

\begin{tabular}{|c|c|c|c|}
\hline \multicolumn{4}{|c|}{ Bahn ticket, only winners } \\
\hline \multirow{2}{*}{ Altruist(-) or protest } & \multicolumn{2}{|c|}{ Vote } & \multirow{2}{*}{ Total } \\
\hline & No & Yes & \\
\hline No & 9 & 239 & 248 \\
\hline Yes & 23 & 100 & 123 \\
\hline Total & 32 & 339 & 371 \\
\hline
\end{tabular}

Losers' savings do not cover the ticket price, and they neither visit other people using the ticket nor mention leisure/work usage. Winners' savings cover the ticket price.

the opportunity to buy it privately. Remarkably, $23 \%$ to $27 \%$ of those who voted in favor of a ticket would not be willing to buy it privately for the same price. Taken together, $15 \%$ to $23 \%$ of the respondents voted differently as citizens than they would have chosen as private consumers.

We next analyze those votes which are not in line with pocketbook considerations. We conjecture that social preferences and expressive motives explain most of these. To test this conjecture in Dataset I we use the variables altruist $(+)$ and altruist $(-)$. These variables describe students who stated that they cared about other students' benefits in their votes and at the same time thought that students on average would gain or lose, respectively, when the ticket was introduced. As seen in Table 7, the majority of respondents who voted in favor of the ticket even if they lost privately thought that other students gained from it and reported that they cared about this gain. Among those who voted against the ticket, even if it promised them higher private savings than the price of the ticket, a clear majority was either of the view that other students would lose from the ticket or mentioned protest motives regarding price or pricing policy in the questionnaire's write-in section.

In Dataset II, we calculated the fraction of those who voted in favor of each ticket but would not buy it privately who also reported at least one social or expressive motive. Here, we consider a social or expressive motive to be present if the respondent stated that an item was at least somewhat important for his or her decision. The motives that we consider relate to altruistic concerns toward other students and to common good considerations. The former are relevant when a respondent notes that savings to others are a motivation to vote in favor of a ticket. 
Table 8: Voting in favor but unwilling to buy: importance of social preferences

\begin{tabular}{lccc}
\hline \hline & Train & Bus & Culture \\
\hline Social preferences or expressive motives & 235 & 147 & 147 \\
Neither social preferences nor expressive motives & 21 & 10 & 0 \\
\hline Total & 256 & 157 & 147 \\
\hline \hline
\end{tabular}

The social preferences and expressive motives considered include for all three tickets savings to other students. They also include environmental aspects for the train ticket, strengthening local public transportation for the bus ticket, and strengthening local cultural life and the belief that others should visit cultural institutions more frequently for the culture ticket.

The latter is present when a respondent supports the service in question because it benefits the environment (in the case of the train ticket), because he or she wants to strengthen local culture or the bus system, or states, paternalistically, that other students should use cultural services more often. ${ }^{9}$ Table 8 shows that everyone who supported the culture ticket without being willing to buy it privately and more than $90 \%$ of those voting in favor of the train or bus ticket despite not being willing to buy it privately claimed at least one social or expressive motive.

Figure 4 summarizes our findings. It shows that $77 \%$ to $87 \%$ of all votes can be rationalized by pocketbook voting alone. In Dataset I this corresponds to losers voting against the ticket and winners voting in favor (see Table 7). Here, we disregard the middle category since it is not clear whether these respondents gain or lose from the ticket. In Dataset II a vote is rationalized by pocketbook considerations if the respondent votes in favor of a ticket if and only if he or she would buy it privately at the price charged (see Table 6). Almost all of the votes that cannot be rationalized in this way can be rationalized by social preferences or expressive motives. The shares of unrationalizable no votes are between one and two percent, and the shares of unrationalizable yes votes are between zero and three percent. ${ }^{10}$

When interpreting Figure 4, it is notable that individual financial interests and

\footnotetext{
${ }^{9}$ Strengthening local cultural institutions or the bus system can also be self-interested, to improve the choices that one has as a private consumer. Similarly, wanting other students to consume more culture might also reflect a desire to have more company at cultural events. Yet another reason for supporting the culture ticket could be related to problems of self-control. Students may want to commit themselves to consuming more culture, just as a flat rate gym membership can be seen as a commitment device to exercise more often (DellaVigna and Malmendier, 2006). Note, however, that buying this ticket privately would also provide a commitment device. The fact that a substantial number of respondents voting in favor of the ticket would not make this private purchase but at the same time state that others should attend cultural events more often suggests that most students saw the self-control problem in their fellow students rather than in themselves.

${ }^{10} \mathrm{We}$ also performed the analysis summarized in Figure 4, splitting the sample by gender and by political orientation. We find that the differences between men and women and between supporters of the left and those of the right are minor.
} 
Figure 4: Rationalizing votes - Datasets I and II

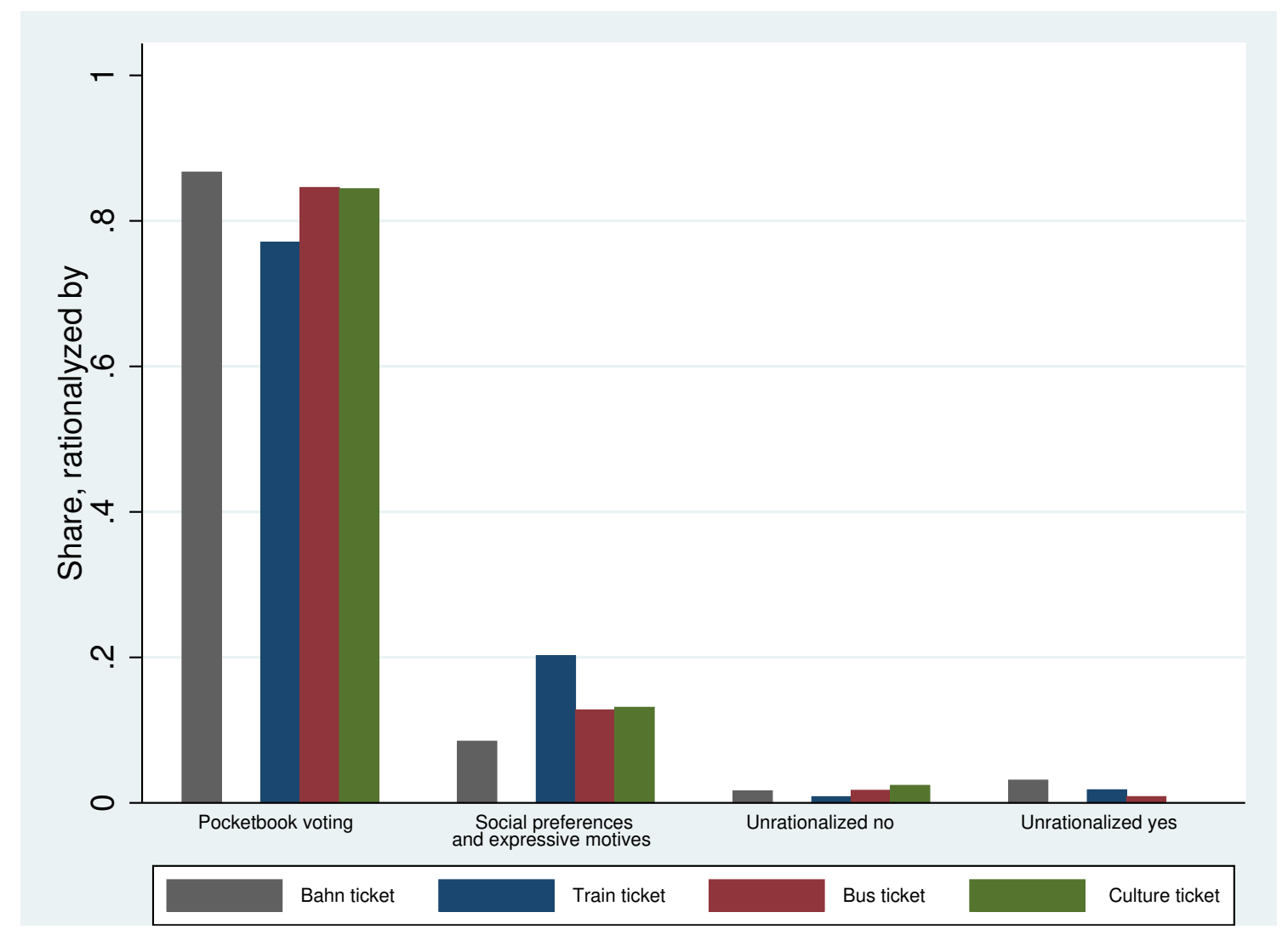

social preferences are not mutually exclusive but, rather, may coincide. Therefore, the figure does not state that around $80 \%$ of all voters would base their decisions only on their own financial benefit. Rather, it shows that there are very few votes that cannot be rationalized by either pocketbook voting or social or expressive considerations or these types of motivations together.

To observe the full power of social preferences or expressive motives, note in Table 6 that although only a minority of students in the sample would have bought the culture ticket or the bus ticket, a majority supported them in the referenda. As Figure 4 shows, for a sizable minority of the voters, social preferences or expressive motives were the decisive factor in their decision. The closeness of the results in the actual referenda on the culture and bus tickets suggests that such motives were pivotal in the former vote and close to pivotal in the latter.

The evidence presented in this section is consistent with an explanation based on pocketbook voting combined with social preferences or expressive motives or both. However, combining insights from our theory, Figure 4, and the election outcomes 
sheds light on the relative plausibility of social preferences and expressive motives as explanations. We first note that two referenda (bus and culture) were close and two referenda (both train tickets) were decided by a large margin. Voters with rational expectations should therefore have considered their likelihood of being pivotal to be much larger in the former votes than in the latter. This conclusion arises also if students form their expectations retrospectively, as approval rates are remarkably stable over time. ${ }^{11}$ In a trade-off between pocketbook benefits and expressive motives, pocketbook considerations are weighted by the probability to change the outcome, and should therefore become less important if this probability is low (see Proposition 1(iii)). In contrast, in a trade-off between pocketbook benefits and social preferences, this probability cancels out, and hence should not affect the relative importance of these motives (see Corollary 1). The finding that pocketbook voting explains around $80 \%$ of votes in each election, irrespective of whether it was close or not, suggests that social preferences play a bigger role than expressive concerns.

\subsection{The turnout decision}

We next analyze how the decision to participate in the vote is related to savings when visiting parents. Figure 5 presents turnout separately for three groups, defined above in Figure 2, according to the monetary gains conferred by the ticket: those who lost from the ticket; those with moderate savings who may also have netted personal gain from trips other than those to visit parents; and those whose savings from visiting parents exceeded the price. Those with zero and moderate savings are least likely to vote; after that, turnout increases monotonically. As predicted by Proposition 2(i), this finding suggests that those with higher stakes are more likely to vote, in line with the rational calculus of voting. It is noteworthy that gains and losses are asymmetrically distributed: the maximum loss is the price of a ticket (84.48 euros), while among the winners, the average savings just from visiting parents is 586 euros. This asymmetry explains why turnout is substantially higher among winners.

\section{The vote}

\subsection{Estimating the voting decision}

We now describe how we arrive from equation (1) at an equation which can be estimated. For the empirical analysis, we assume that the weights in the utility

\footnotetext{
${ }^{11}$ Before the ticket was split in two in 2010, the train ticket had been approved by $80 \%$ in 2008 and $84 \%$ in 2009. In the referenda in 2011 and 2012, the approval rates were $80 \%$ and $82 \%$. The culture ticket passed only narrowly in 2012 with a $51 \%$ share of yes votes.
} 
Figure 5: Turnout and savings - Dataset I

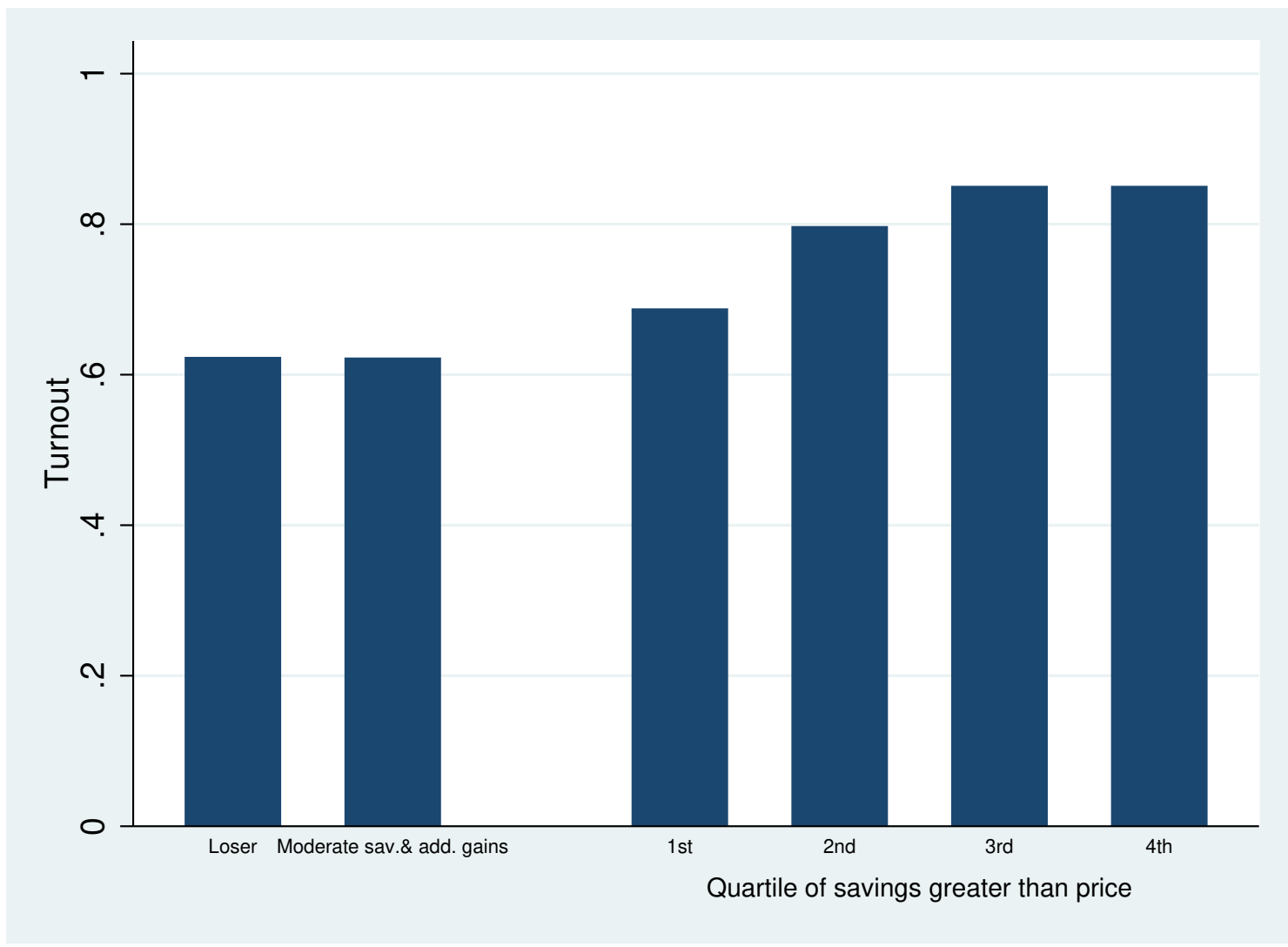

Losers' savings do not cover the ticket price, and they neither visit other people using the ticket nor mention leisure/work usage. For the middle group, the savings when visiting parents do not cover the ticket price; however, they mention other trips.

function and the perceived probability of changing the outcome are the same for all individuals, $\lambda_{i}=\lambda, \boldsymbol{\zeta}_{i}=\boldsymbol{\zeta}, \boldsymbol{\psi}_{i}=\boldsymbol{\psi}$, and $p_{i}=p$ for all voters $i$. Moreover, in line with Card et al. (2012) we allow for an additive impact of a vector of individual covariates $\mathbf{x}_{i}$ such as gender, political affiliation or fields of study, with the vector $\boldsymbol{\rho}$ measuring the effects of the various covariates. Finally, we add an error term $\mu_{i}$ with a standard normal distribution, which also includes any considerations not captured by our model.

Combining these elements, we modify equation (1) to

$$
y_{i}=\beta\left(b_{i}^{1}-b_{i}^{0}\right)+\boldsymbol{\alpha} \cdot\left(\mathbf{a}_{i}^{1}-\mathbf{a}_{i}^{0}\right)+\boldsymbol{\rho} \cdot \mathbf{x}_{i}+\mu_{i} .
$$

Equation (6) is the basis for the empirical analysis, where we estimate the coefficients $\beta, \boldsymbol{\alpha}$ and $\boldsymbol{\rho}$ using a probit regression. Individual $i$ votes in favor of the ticket if and 
only if $y_{i} \geq 0$, that is, $\mu_{i} \geq-\beta\left(b_{i}^{1}-b_{i}^{0}\right)-\boldsymbol{\alpha} \cdot\left(\mathbf{a}_{i}^{1}-\mathbf{a}_{i}^{0}\right)-\boldsymbol{\rho} \cdot \mathbf{x}_{i}$. Making use of the symmetry of the normal distribution, the probability that $i$ votes in favor of the ticket is given by $\Phi\left(\beta\left(b_{i}^{1}-b_{i}^{0}\right)+\boldsymbol{\alpha} \cdot\left(\mathbf{a}_{i}^{1}-\mathbf{a}_{i}^{0}\right)+\boldsymbol{\rho} \cdot \mathbf{x}_{i}\right)$, where $\Phi$ is the cumulative distribution function of the standard normal distribution.

The estimated coefficient $\beta=p \lambda$ measures the impact of pocketbook motives on the gain from switching from a negative to a positive vote. The coefficients $\boldsymbol{\alpha}=p \boldsymbol{\zeta}+\boldsymbol{\psi}$ are the joint impact of instrumental social preferences and expressive motives on this gain. Note, however, that due to the standardization of the error term inherent in the probit model only the relative sizes of the coefficients can be interpreted.

We cannot observe the exact monetary benefit $b_{i}^{1}-b_{i}^{0}$ since we do not have information about the shape of the function $w_{i}$ and the counterfactual intensity of use in case the ticket is not provided. In Dataset I (see Section 4.2), we have a continuous variable for the savings $\tau h_{i}^{1}$ provided by the ticket, measured by what would have been the price of the trips that were actually taken with the ticket in the absence of the ticket. We use savings net of the ticket price, $\tau h_{i}^{1}-t$, to approximate ${ }^{12}$ the equivalent variation by

$$
b_{i}^{1}-b_{i}^{0}=\left\{\begin{array}{cl}
\ln \left(\tau h_{i}^{1}-t+1\right) & \text { if } \tau h_{i}^{1}-t \geq 0 \\
\tau h_{i}^{1}-t & \text { if } \tau h_{i}^{1}-t<0 .
\end{array}\right.
$$

By using net savings directly we would risk overestimating the benefit which heavy users derive from the ticket, since individual use might drop considerably if marginal cost were positive instead of zero. Therefore, we use the logarithm of net savings in the empirical analysis for individuals whose savings exceed the ticket price. Here, we add +1 to ensure that net savings smaller than one are valued positively. In contrast, since negative net savings occur only with individuals who use the ticket rarely or not at all, we do not transform negative net savings. In the empirical application, we prefer not to force a common coefficient on the two branches of equation (7), and hence introduce both of them as separate regressors.

In Dataset II, the pocketbook benefit is quantified by categorical variables. The coefficients associated with these variables measure by how much the utility of a voter whose intensity of use of the service covered by the ticket falls in the respective category exceeds the utility of a voter who never uses it. ${ }^{13}$

Social preferences and expressive motives are measured by survey responses which are coded in binary variables. In all cases, these variables state whether

\footnotetext{
${ }^{12}$ See the Appendix A.III for a detailed discussion of this approximation.

${ }^{13}$ Formally, this amounts to approximating the linear function $\beta\left(b_{i}^{1}-b_{i}^{0}\right)$ by a step function. The coefficients estimate the vertical jumps from the lowest category "never" to the various categories of more intensive use, rather that a unique slope $\beta$.
} 
an individual cares for some issue linked to the outcome of the vote, such as benefits of others or the environment. In Dataset I, we use the variables altruist $(+)$, altruist(-), and protest, as explained in Section 4.2. In Dataset II, we code an individual as having a certain social or expressive motive if he or she mentioned, in the four-point Likert scale used in the survey (see Section 4.3), that the corresponding item is important. Thus, in the notation from above, for a voter who states (does not state) that an issue is important, the corresponding component of the vector $\mathbf{a}_{i}^{1}-\mathbf{a}_{i}^{0}$ is set to one (zero). The estimated coefficient then gives the aggregate effect of social and expressive benefits of an individual who states that he or she cares for the specific issue. Notice that the estimated social or expressive valuation of a ticket may be negative not only if an individual thinks that it is not valued by other students, but also if the individual has libertarian reservations against forcing a collective choice upon individuals. Such libertarian concerns may be social or expressive or a combination of the two, just as with arguments in favor of the ticket.

\subsection{Empirical results}

We now turn to the regression analysis of the voting decisions. The dependent variable is the probability of voting in favor of the respective ticket, which we estimate using probit models. We report results for Dataset I in Table 9. The main explanatory variables measuring monetary self-interest are log pos. net savings and neg. net savings, which refer to the two branches of equation (7). The table shows the corresponding marginal effects for the benchmark student who is defined by all indicator variables being zero. However, to account for the high variation with respect to individual savings on trips to visit parents, we also incorporate the individual values of the net savings variable in the calculation of marginal effects. Hence, we display average marginal effects for benchmark students. ${ }^{14}$

The variable $\log$ pos. net savings shows the expected positive sign and is significant at the 0.1 percent level. From column (2) onwards, the corresponding marginal effect remains virtually the same if we include additional variables. This implies that a benchmark student is on average 0.6-0.7 percentage points more likely to vote in

\footnotetext{
${ }^{14}$ We calculate marginal effects as discrete changes from zero to one for all indicator variables. For positive net savings, average marginal effects are determined as follows. Setting all indicator variables to zero, we first evaluate the derivative of the probability to vote in favor of the ticket with respect to the log of net savings +1 at the individual value of net savings of each observation with positive net savings. These derivatives are then averaged over all observations with positive net savings in the sample, yielding the average marginal effects displayed in the first row of Table 9 . Similarly, for observations with negative net savings we evaluate the derivative of the probability to vote for the ticket with respect to net savings at their individual values and average over these observations, yielding the average marginal effects in the second row of Table 9. Coefficients for all regressions are reported in Tables A.1-A.6 in the supplementary material.
} 
Table 9: Bahn ticket - Dataset I

\begin{tabular}{|c|c|c|c|c|c|}
\hline \multicolumn{6}{|c|}{ Dependent Variable: Supporting Bahn Ticket } \\
\hline & (1) & $(2)$ & $(3)$ & $(4)$ & (5) \\
\hline Log pos. net savings & $\begin{array}{c}0.028^{* * *} \\
(4.85)\end{array}$ & $\begin{array}{c}0.071^{* * *} \\
(5.59)\end{array}$ & $\begin{array}{c}0.073^{* * *} \\
(5.15)\end{array}$ & $\begin{array}{c}0.066^{* * *} \\
(3.78)\end{array}$ & $\begin{array}{c}0.062^{* * *} \\
(3.64)\end{array}$ \\
\hline Neg. net savings & $\begin{array}{c}0.0022^{*} \\
(1.98)\end{array}$ & $\begin{array}{c}0.0002 \\
(0.18)\end{array}$ & $\begin{array}{c}0.0005 \\
(0.52)\end{array}$ & $\begin{array}{l}0.0014 \\
(1.48)\end{array}$ & $\begin{array}{c}0.0016 \\
(1.57)\end{array}$ \\
\hline Leisure/work & & $\begin{array}{l}0.073 \\
(0.97)\end{array}$ & $\begin{array}{l}0.030 \\
(0.39)\end{array}$ & $\begin{array}{l}0.030 \\
(0.39)\end{array}$ & $\begin{array}{l}0.034 \\
(0.44)\end{array}$ \\
\hline Visiting others & & $\begin{array}{c}0.304^{* * *} \\
(8.83)\end{array}$ & $\begin{array}{c}0.313^{* * *} \\
(8.67)\end{array}$ & $\begin{array}{c}0.279 * * * \\
(6.86)\end{array}$ & $\begin{array}{c}0.281^{* * *} \\
(6.99)\end{array}$ \\
\hline Female & & & $\begin{array}{l}0.055 \\
(1.57)\end{array}$ & $\begin{array}{l}0.037 \\
(0.98)\end{array}$ & $\begin{array}{l}0.035 \\
(0.94)\end{array}$ \\
\hline Freshman & & & $\begin{array}{l}0.079 \\
(1.58)\end{array}$ & $\begin{array}{l}0.106^{*} \\
(2.01)\end{array}$ & $\begin{array}{c}0.114^{*} \\
(2.18)\end{array}$ \\
\hline Altruist $(-)$ & & & & $\begin{array}{c}-0.143^{* *} \\
(-2.98)\end{array}$ & $\begin{array}{c}-0.140^{* *} \\
(-2.88)\end{array}$ \\
\hline Altruist $(+)$ & & & & $\begin{array}{c}0.283^{* * *} \\
(6.66)\end{array}$ & $\begin{array}{c}0.281^{* * *} \\
(6.70)\end{array}$ \\
\hline Protest & & & & & $\begin{array}{c}-0.080^{*} \\
(-1.96)\end{array}$ \\
\hline Pseudo $\mathrm{R}^{2}$ & 0.232 & 0.321 & 0.344 & 0.438 & 0.442 \\
\hline Log likelihood & -392.2 & -344.4 & -302.2 & -234.5 & -232.6 \\
\hline Observations & 818 & 810 & 741 & 669 & 669 \\
\hline
\end{tabular}

Probit estimation; marginal effects for benchmark students; discrete changes from 0 to 1 for indicator variables; $\mathrm{z}$-statistic in parentheses. ${ }^{*} p<0.05,{ }^{* *} p<0.01,{ }^{* * *} p<0.001$.

favor if net savings increase by $10 \%$. Given the range of the variable, this translates into sizable differences in the prediction: based on the full specification, column (5), the probability of a positive vote is $32 \%$ if the benchmark student's savings only cover the ticket price. The predicted probability is $71 \%$ if his net savings are of average size and $82 \%$ if he saves one thousand euros more than the ticket price. The marginal effect of the variable neg. net savings is not statistically significantly but positive throughout. The lack of statistical significance may be due to the fact that this variable varies only between -84.48 and zero euros. Finally, visiting others using the ticket significantly increases the probability of voting in favor. These findings confirms the high importance of personal monetary benefits to individual votes. Hence, for the observations contained in Dataset I, we clearly reject null-hypothesis H1, which says that pocketbook motives do not influence the voting decision.

However, social preferences and/or expressive concerns also play a role in this vote: both altruism variables carry the expected sign and are significant at least at the 1 percent level. Approximately half of the students consider their fellow students' gains and losses in their votes. According to their own perception of whether the other students will on average gain or lose, these students are, ceteris 
paribus, respectively more or less likely than the benchmark to vote in favor of the ticket. Expecting other students to gain from the ticket and considering this expectation increases support for the ticket as much as using it oneself to visit other people aside from one's parents. Furthermore, the protest variable carries a negative sign and is significant at the 5 percent level. This suggests that some students protested against the train company's pricing policy by voting against the ticket. From these results we conclude that null-hypothesis $\mathbf{H 2}$, which states that neither social nor expressive motives affect the voting decision, is rejected for Dataset I.

To examine whether general political attitudes contribute to explaining individual votes, we include party preferences in the regressions. This does not change our main results. All else being equal, supporters of the left are not more likely to vote in favor of the ticket; we do not find significant effects for any of the parties (Table A.7 in the supplementary material). This finding also holds true when grouping parties on the left (Social Democrats, Greens, and Left Party) and on the right (Christian Democrats and Liberal Democrats) into blocs.

We summarize the quantitative results on the voting decision in Figure 6, based on the specification of column (5) in Table 9. In this figure, we restrict attention to monetary savings and altruism since these variables are our main focus of interest. Moreover, without placing too much emphasis on this, it is noted that the private savings and altruism variables particularly contribute to the pseudo R-squared in Table 9 .

The solid red line in Figure 6 plots the predicted probability of voting in favor of the Bahn ticket for a benchmark student as a function of net savings. This student's predicted probability of voting yes reaches $50 \%$ at net savings of 10 euros, which is plausible since students with very small net gains should be fairly indifferent between the alternatives. The broken blue line labelled $\operatorname{Pr}($ altruist $(+))$ shows that the predicted probability to support the ticket is shifted upwards by a substantial amount when the respondent cares about the benefit of others and anticipates that other students benefit from the ticket. Even when he does not use the ticket at all, such a student is more likely to support the ticket in the referendum than to reject it. The dotted line labelled $\operatorname{Pr}($ altruist(-)) shows the predicted voting behavior of an altruist who estimates that the ticket is harmful to the interests of others. Even with substantial net savings of 140 euros, such a student is more likely to vote against the ticket than in favor of it.

We present the results for the train, bus and culture tickets in Tables 10, 11 and 12, respectively. To interpret the results right away, we display the marginal effects for the benchmark students in the regression tables. These students are characterized by all indicator variables being zero. Thus, the benchmark is male and not a freshman, and savings to other students are not important to his decision. 
Figure 6: Predicted probability to vote in favor of Bahn ticket - Dataset I

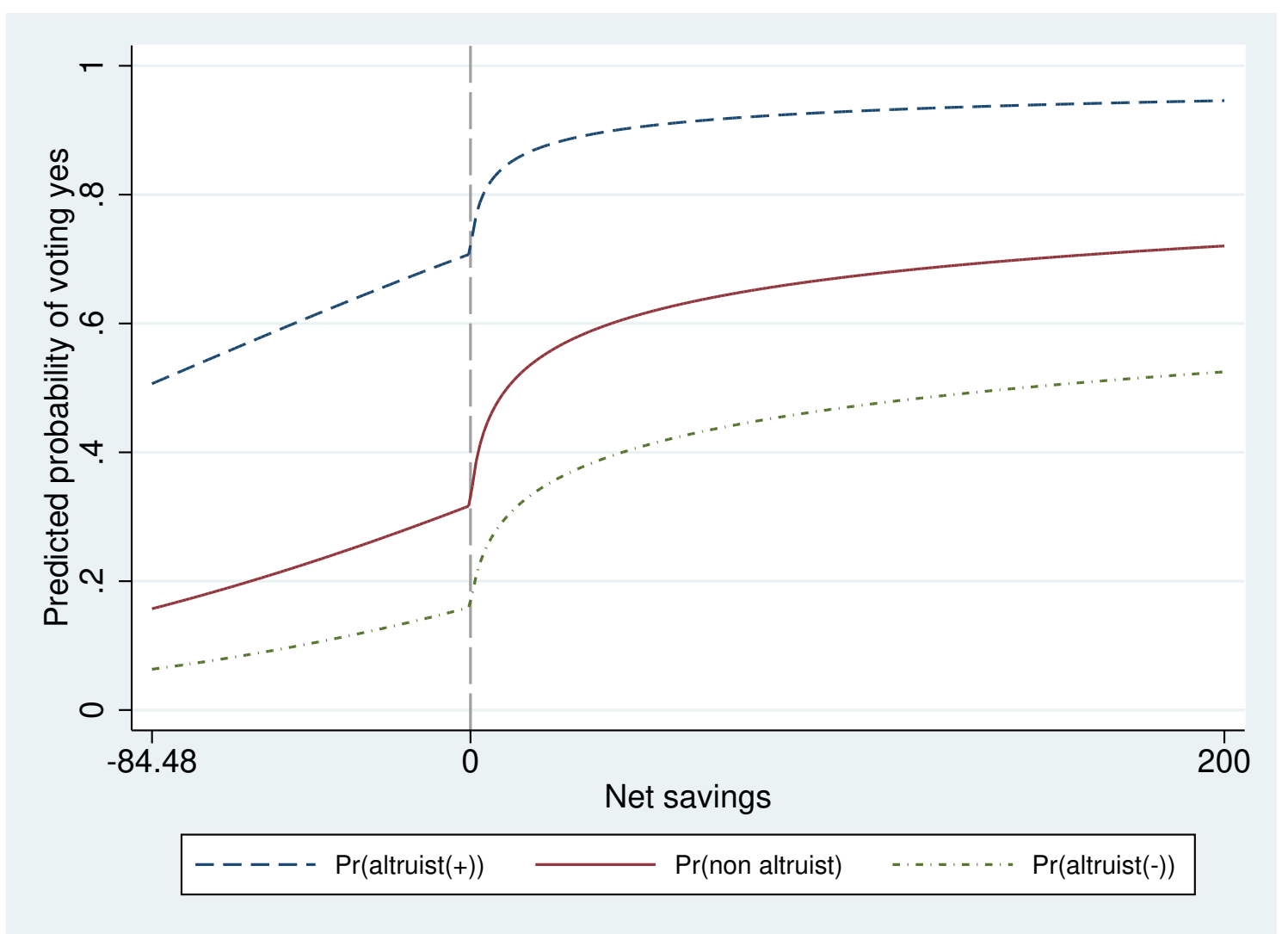

$\diamond$ Prediction based on Table 9 , column $(5)$, as function of net savings in euros. All binary variables except altruist $(+)$
and altruist $(-)$ are set to zero. The line labelled $\operatorname{Pr}($ non altruist) refers to an individual who states that he did
not consider the benefits of others in his vote. The line $\operatorname{Pr}($ altruist $(+))$ ( $\operatorname{Pr}($ altruist $(-)))$ refers to an individual
who considered benefits of others in his vote and estimates that others on average gain (lose) from the Bahn ticket.

The base category for the intensity of use is "never".

Our econometric results confirm the impressions gathered in Section 5: the probability of voting in favor of a ticket strongly increases with the intensity of personal use, suggesting a high degree of pocketbook voting. The effects are significant at the 0.1 percent level and of an economically relevant size. For example, concerning the bus ticket, estimations imply that an otherwise identical student who uses the bus several times per week is more than 70 percentage points more likely to vote in favor of this ticket than the benchmark student who does not use the bus. Thus, also for Dataset II, null-hypothesis $\mathbf{H 1}$ is rejected.

However, variables capturing social preferences and/or expressive concerns also show highly significant and positive effects. Those who consider savings to other 
Table 10: Train ticket - Dataset II

\begin{tabular}{|c|c|c|c|c|}
\hline \multicolumn{5}{|c|}{ Dependent Variable: Supporting Train Ticket } \\
\hline & $(1)$ & $(2)$ & $(3)$ & $(4)$ \\
\hline Rarely $(\leq 5 /$ year $)$ & $\begin{array}{c}0.428^{* * *} \\
(7.35)\end{array}$ & $\begin{array}{c}0.389^{* * *} \\
(6.36)\end{array}$ & $\begin{array}{c}0.393^{* * *} \\
(6.73)\end{array}$ & $\begin{array}{c}0.373^{* * *} \\
(6.21)\end{array}$ \\
\hline Sometimes (monthly) & $\begin{array}{c}0.657^{* * * *} \\
(13.05)\end{array}$ & $\begin{array}{c}0.648^{* * *} \\
(11.72)\end{array}$ & $\begin{array}{c}0.680^{* * *} \\
(13.56)\end{array}$ & $\begin{array}{c}0.676^{* * *} \\
(13.14)\end{array}$ \\
\hline Often (weekly) & $\begin{array}{c}0.701^{* * *} \\
(14.10)\end{array}$ & $\begin{array}{c}0.698 * * * \\
(12.45)\end{array}$ & $\begin{array}{c}0.749 * * * \\
(14.61)\end{array}$ & $\begin{array}{c}0.745^{* * *} \\
(14.19)\end{array}$ \\
\hline Very often $(\geq 2 /$ week $)$ & $\begin{array}{c}0.709 * * * \\
(14.39)\end{array}$ & $\begin{array}{c}0.709 * * * \\
(12.70)\end{array}$ & $\begin{array}{c}0.766^{* * *} \\
(15.19)\end{array}$ & $\begin{array}{c}0.760 * * * \\
(14.69)\end{array}$ \\
\hline Female & & $\begin{array}{c}0.119 * * \\
(3.01)\end{array}$ & $\begin{array}{c}0.090^{*} \\
(2.44)\end{array}$ & $\begin{array}{c}0.074^{*} \\
(2.04)\end{array}$ \\
\hline Freshman & & $\begin{array}{c}-0.075^{*} \\
(-2.10)\end{array}$ & $\begin{array}{l}-0.043 \\
(-1.39)\end{array}$ & $\begin{array}{l}-0.029 \\
(-0.92)\end{array}$ \\
\hline Savings to others & & & $\begin{array}{c}0.285^{* * * *} \\
(5.48)\end{array}$ & $\begin{array}{c}0.198^{* * * *} \\
(3.65)\end{array}$ \\
\hline Environment & & & & $\begin{array}{c}0.242^{* *} \\
(3.25)\end{array}$ \\
\hline Pseudo $\mathrm{R}^{2}$ & 0.285 & 0.297 & 0.342 & 0.364 \\
\hline Log likelihood & -356.2 & -334.4 & -298.1 & -277.9 \\
\hline Observations & 1247 & 1191 & 1163 & 1145 \\
\hline
\end{tabular}

students important to their decisions vote in favor of the respective ticket with a higher probability. Additionally, students who consider environmental aspects or strengthening local public transportation to be important are more likely to vote in favor of the train ticket or the bus ticket, respectively. The same holds true regarding the culture ticket for those who indicate that strengthening local cultural life or encouraging others to visit these institutions more frequently is important to them in their voting decisions. ${ }^{15}$ From these results, null-hypothesis $\mathbf{H 2}$ is also rejected in Dataset II.

Freshmen are, ceteris paribus, more likely to vote in favor of the bus and culture tickets. However, because for freshmen, the questions on the intensity of use refer to a shorter time period or might cover some time when they were not yet in Goettingen, we refrain from emphasizing this finding.

As a robustness check, we also control for the field of study (Table A.8 in the

\footnotetext{
${ }^{15} \mathrm{~A}$ similar picture emerges from the regression analysis if we use indicator variables encompassing motives that were at least somewhat important rather than focusing on motives that were important. In line with expectations, these variables capturing less pronounced social preferences or expressive concerns in general display smaller marginal effects than those shown in Tables 1012. We have also included complete sets of indicator variables containing information on whether someone considered a motive unimportant, somewhat important, or important, and the conclusions remained the same.
} 
Table 11: Bus ticket - Dataset II

\begin{tabular}{lcccc}
\hline \multicolumn{4}{c}{ Dependent Variable: Supporting Bus Ticket } \\
\hline Rarely (1 or 2/semester) & $0.241^{* * *}$ & $0.237^{* * *}$ & $0.217^{* * *}$ & $0.203^{* * *}$ \\
& $(6.94)$ & $(6.67)$ & $(6.15)$ & $(5.64)$ \\
Sometimes (monthly) & $0.415^{* * *}$ & $0.439^{* * *}$ & $0.416^{* * *}$ & $0.411^{* * *}$ \\
& $(9.81)$ & $(9.84)$ & $(8.79)$ & $(8.19)$ \\
Often (weekly) & $0.597^{* * *}$ & $0.613^{* * *}$ & $0.617^{* * *}$ & $0.587^{* * *}$ \\
& $(14.88)$ & $(14.45)$ & $(13.58)$ & $(11.38)$ \\
Very often ( $\geq 2 /$ week) & $0.706^{* * *}$ & $0.726^{* * *}$ & $0.744^{* * *}$ & $0.728^{* * *}$ \\
& $(27.55)$ & $(26.15)$ & $(24.39)$ & $(20.17)$ \\
Female & & -0.001 & -0.006 & 0.007 \\
Freshman & & $(-0.04)$ & $(-0.27)$ & $(0.29)$ \\
Savings to others & & $0.079^{* *}$ & $0.081^{* *}$ & $0.067^{*}$ \\
Strengthening bus system & & $(2.75)$ & $(2.92)$ & $(2.44)$ \\
& & & $0.204^{* * *}$ & $0.158^{* * *}$ \\
& & & $(5.36)$ & $(4.07)$ \\
Pseudo R ${ }^{2}$ & & & $0.418^{* * *}$ \\
Log likelihood & 0.224 & 0.232 & 0.252 & 0.285 \\
Observations & -668.1 & -629.3 & -589.1 & -539.1 \\
\hline \hline
\end{tabular}

Probit estimation; discrete effects for benchmark students due to changes from 0 to 1 for all variables; z-statistic in parentheses. ${ }^{*} p<0.05,{ }^{* *} p<0.01,{ }^{* * *} p<0.001$.

Table 12: Culture ticket - Dataset II

\begin{tabular}{lcccc}
\hline \hline \multicolumn{4}{c}{ Dependent Variable: Supporting Culture Ticket } \\
\hline & $(1)$ & $(2)$ & $(3)$ & $(4)$ \\
\hline Rarely (1 or 2/year) & $0.376^{* * *}$ & $0.389^{* * *}$ & $0.355^{* * *}$ & $0.290^{* * *}$ \\
& $(11.67)$ & $(11.18)$ & $(9.60)$ & $(6.83)$ \\
Sometimes (3 to 5/year) & $0.578^{* * *}$ & $0.611^{* * *}$ & $0.597^{* * *}$ & $0.572^{* * *}$ \\
& $(18.57)$ & $(15.83)$ & $(13.28)$ & $(7.80)$ \\
Often (6 to 10/year) & $0.634^{* * *}$ & $0.687^{* * *}$ & $0.693^{* * *}$ & $0.649^{* * *}$ \\
& $(17.78)$ & $(14.68)$ & $(12.09)$ & $(4.94)$ \\
Very often (> 10/year) & $0.605^{* * *}$ & $0.675^{* * *}$ & $0.640^{* * *}$ & $0.522^{* *}$ \\
& $(13.59)$ & $(12.42)$ & $(7.96)$ & $(2.95)$ \\
Female & & $0.073^{* *}$ & $0.071^{*}$ & 0.019 \\
& & $(2.65)$ & $(2.53)$ & $(0.80)$ \\
Freshman & & $0.101^{* *}$ & $0.109^{* *}$ & $0.084^{*}$ \\
& & $(3.01)$ & $(3.17)$ & $(2.58)$ \\
Savings to others & & & $0.262^{* * *}$ & $0.104^{*}$ \\
& & & $(6.21)$ & $(2.40)$ \\
Others should go & & & & $0.433^{* * *}$ \\
& & & & $(5.83)$ \\
Strengthening local culture & & & & $(8.74)$ \\
\hline Pseudo $\mathrm{R}^{2}$ & 0.192 & 0.191 & 0.215 & 0.440 \\
Log likelihood & -664.7 & -633.7 & -571.1 & -378.7 \\
Observations & 1189 & 1131 & 1055 & 988 \\
\hline \hline
\end{tabular}

Probit estimation; discrete effects for benchmark students due to changes from 0 to

1 for all variables; $\mathrm{z}$-statistic in parentheses. ${ }^{*} p<0.05,{ }^{* *} p<0.01,{ }^{* * *} p<0.001$ 
supplementary material). The overall picture is that it does not appear to matter much for individual votes. Only a small number of fields show significant effects on the voting decisions, and our main results remain robust. All else being equal, students of the humanities and social sciences are more likely to vote in favor of the culture ticket. One explanation for this finding may be the large share of students in these fields who study culture-related subjects.

The strong empirical support for both pocketbook voting and social or expressive considerations is robust to the inclusion of party preferences and to the inclusion of both fields and party preferences in the regressions (Tables A.9 and A.10 in the supplementary material). Furthermore, support for the train and bus tickets appears quite unrelated to party preferences. ${ }^{16}$ However, we find that support for the culture ticket, ceteris paribus, is larger among supporters of parties on the left. The qualitative results with respect to pocketbook voting and social or expressive considerations remain unchanged also if we group parties into a left bloc and a right bloc. The only statistically significant effect of party blocs is that supporters of the left are more likely to vote in favor of the culture ticket.

A general concern with survey data is the reliability of answers. In our setting, this problem may arise particularly with regard to social preferences because of social desirability. For example, one might wonder whether the respondents genuinely care for others or if they just feel social pressure to state this concern. However, both the descriptive and econometric analyses show that stated social preferences, altruism in particular, or expressive concerns have a substantial impact on the voting decision, explaining most votes that cannot be rationalized by monetary benefits.

From an econometric perspective, one might be concerned with reverse causality, omitted variables, or sample selection. A reverse causality problem would arise if those respondents who voted in favor of a ticket against their monetary interest ex post rationalized their decision by mentioning social preferences or expressive concerns. However, this argumentation leaves open the question of why they voted in favor of the ticket in the first place. Given the extensive set of controls that we use, there is no obvious candidate for an omitted variable that affects the voting decision and is correlated with the explanatory variables. An issue of sample selection could arise if the respondents in the surveys systematically differ from the student population. As shown in Section 4, voters are somewhat overrepresented in Dataset I. This is not, however, a problem because we exclude nonvoters from the analysis of voting decisions. Finally, semester tickets should only play a minor role in the decision whether to enroll at University of Goettingen. Taken together, we do not think that any of these issues is likely to seriously bias our results.

\footnotetext{
${ }^{16}$ The only statistically significant effect we find is that Liberal Democrats are less likely to support the train ticket (see Table A.10).
} 
Summarizing our empirical analysis so far, we find that in both datasets, the null-hypotheses $\mathbf{H 1}$ and $\mathbf{H} \mathbf{2}$ are rejected. Thus, pocketbook voting is an important determinant of referendum outcomes, but social preferences or expressive concerns also have measurable and sizeable effects on the voting decision. As explained in Section 3.1, we cannot determine which of these two motives is prevalent; they are observationally equivalent. We can, however, conclude that voting is not purely expressive. The strong impact of monetary benefits on voting shows that either voters assigned a non-negligible probability to their being decisive in the referendum, as in the bottom half of Table 2(b), or expressive motives are absent and altruism and protest motives are necessarily instrumental, as in the right half of Table 2(a).

\section{Participation}

We now turn to the second part of our analysis, which is to understand what induced students to vote in the referendum. For this purpose, we use Dataset I, which also contains responses by non-voters.

\subsection{Estimating the participation decision}

For the estimation, we assume as before that marginal utilities and perceived probabilities to affect the referendum outcome are the same for all individuals, $\lambda_{i}=\lambda$, $\boldsymbol{\zeta}_{i}=\boldsymbol{\zeta}, \boldsymbol{\psi}_{i}=\boldsymbol{\psi}, p_{i}^{1}=p^{1}$, and $p_{i}^{0}=p^{0}$ for all $i$. Moreover, we use (7) to quantify $b_{i}^{1}-b_{i}^{0}$, we measure $\mathbf{a}_{i}^{1}-\mathbf{a}_{i}^{0}$ by the same categorical variables as in the regression on the decision how to vote, and we introduce the same controls $\mathbf{x}_{i}$ and an error term $\nu_{i}$ with a standard normal distribution. In the participation equation, the controls also capture $d_{i}-c_{i}$, the net benefit from voting which is unrelated to how one votes.

There are two alternative measures for the indicators of the intended vote. First, a natural measure is provided by the actual vote of those who participated, and the self-reported hypothetical vote of those who did not participate. We capture this information by two indicators $I_{i}^{a 1}$ and $I_{i}^{a 0}$. The indicator $I_{i}^{a 1}\left(I_{i}^{a 0}\right)$ equals one if the individual voted or would have voted yes (no), and zero otherwise. However, the stated hypothetical vote of non-participants might differ from the vote they would have cast had they actually voted. In contrast to this, we have an objective measure for the monetary benefits. Exploiting this information, we define an indicator $I_{i}^{b 1}$ $\left(I_{i}^{b 0}\right)$ which equals one if the sign of net savings $\tau h_{i}^{1}-t$ is non-negative (negative), and zero otherwise. Relating to pocketbook benefits, we prefer to use this objective indicator. It would, however, be problematic to interact the variables measuring social and expressive motives with an indicator based exclusively on monetary benefits. Therefore, we prefer to attribute the two definitions to the two types of variables 
separately. That is, we interact monetary benefits with the indicators $I_{i}^{b 1}$ and $I_{i}^{b 0}$, while the variables $\mathbf{a}_{i}^{1}-\mathbf{a}_{i}^{0}$ are interacted with $I_{i}^{a 1}$ and $I_{i}^{a 0}$. Thus, we modify equation (5) to

$$
\begin{aligned}
z_{i}=\delta^{1} I_{i}^{b 1} \ln \left(\tau h_{i}^{1}-t+1\right)+\delta^{0} I_{i}^{b 0}\left(t-\tau h_{i}^{1}\right) \\
+\boldsymbol{\gamma}^{1} \cdot\left[I_{i}^{a 1}\left(\mathbf{a}_{i}^{1}-\mathbf{a}_{i}^{0}\right)\right]+\boldsymbol{\gamma}^{0} \cdot\left[I_{i}^{a 0}\left(\mathbf{a}_{i}^{1}-\mathbf{a}_{i}^{0}\right)\right]+\boldsymbol{\sigma} \cdot \mathbf{x}_{i}+\nu_{i} .
\end{aligned}
$$

Equation (8) is the basis of the probit estimation of the participation decision. It yields estimators for the coefficients $\delta^{1}=p^{1} \lambda, \delta^{0}=p^{0} \lambda, \boldsymbol{\gamma}^{1}=p^{1} \boldsymbol{\zeta}+\boldsymbol{\psi}, \boldsymbol{\gamma}^{0}=$ $-\left(p^{0} \boldsymbol{\zeta}+\boldsymbol{\psi}\right)$, and $\boldsymbol{\sigma}$, the impact of controls on the net benefit of participation. In this estimation, for individuals with negative net savings, we use the net loss $\left(t-\tau h_{i}^{1}\right)$ to have a positive coefficient associated with the regressor expressing monetary stakes. Moreover, similar to the analysis of the voting decision, we find only a combined impact of instrumental social preferences and expressive motivations, quantified by the coefficients $\gamma^{1}$ and $\gamma^{0}$.

\subsection{Empirical results}

The results from estimating equation (8) are presented in Table 13, which shows the average marginal effects for benchmark students. ${ }^{17}$ We see a highly significant positive effect from the variable $\log$ pos. stakes, which stands for $I_{i}^{b 1} \ln \left(\tau h_{i}^{1}-t+1\right)$, the monetary benefit of those who have positive net savings from the ticket on trips to parents. This effect is robust to the inclusion of additional motives and control variables. Quantitatively, a 10\% increase in the net gain from the ticket raises the probability of participation by 0.3 to 0.4 percentage points. Using the specification from column (5), for a benchmark student, this results in an increase in the predicted probability of turnout from $54 \%$ at net savings of zero to $80 \%$ at average net savings and to $86 \%$ at net savings of a thousand euros.

The variable neg. stakes, which stands for $I_{i}^{b 0}\left(t-\tau h_{i}^{1}\right)$, the absolute value of the monetary loss inflicted by the ticket, also shows a positive sign. It is insignificant in the first columns but approaches significance when more explanatory variables are included. Similarly to the voting decision, the difference in significance between both stakes variables may be due to the asymmetric distribution of gains and losses: the latter are limited to the yearly price of the ticket, 84.48 euros, whereas the stakes of someone who uses the Bahn ticket every weekend to visit his or her parents could be much higher. In terms of size, the coefficient estimated for negative stakes is again substantial. Based on the specification in column (5) of Table 13, a benchmark

\footnotetext{
${ }^{17}$ As in Section 6, all binary variables are zero for benchmark students. The continuous variables enter into the calculation of marginal effects at individual values.
} 
student whose savings just cover ticket cost is 14 percentage points less likely to participate in the referendum than someone who does not use the ticket at all.

These results are in line with Proposition 2(i) which states that sufficiently high monetary stakes induce an individual to participate in the referendum. As seen from the different significance levels, the empirical support for this statement is, however, stronger for individuals with pocketbook gains than for those with pocketbook losses.

Table 13: Taking part - Dataset I

\begin{tabular}{|c|c|c|c|c|c|}
\hline \multicolumn{6}{|c|}{ Dependent Variable: Taking Part in Referendum } \\
\hline & (1) & $(2)$ & (3) & (4) & (5) \\
\hline Log pos. stakes & $\begin{array}{c}0.029^{* * *} \\
(3.44)\end{array}$ & $\begin{array}{c}0.031^{* * *} \\
(3.56)\end{array}$ & $\begin{array}{c}0.033^{* * *} \\
(3.90)\end{array}$ & $\begin{array}{c}0.035^{* * *} \\
(3.76)\end{array}$ & $\begin{array}{c}0.037^{* * *} \\
(3.81)\end{array}$ \\
\hline Neg. stakes & $\begin{array}{c}0.0003 \\
(0.31)\end{array}$ & $\begin{array}{c}0.0004 \\
(0.51)\end{array}$ & $\begin{array}{c}0.0009 \\
(1.10)\end{array}$ & $\begin{array}{c}0.0014 \\
(1.47)\end{array}$ & $\begin{array}{c}0.0016 \\
(1.65)\end{array}$ \\
\hline Leisure/work & & $\begin{array}{l}-0.012 \\
(-0.21)\end{array}$ & $\begin{array}{l}-0.003 \\
(-0.05)\end{array}$ & $\begin{array}{l}0.035 \\
(0.64)\end{array}$ & $\begin{array}{l}0.035 \\
(0.62)\end{array}$ \\
\hline Visiting others & & $\begin{array}{l}0.006 \\
(0.22)\end{array}$ & $\begin{array}{l}0.006 \\
(0.20)\end{array}$ & $\begin{array}{l}0.010 \\
(0.32)\end{array}$ & $\begin{array}{l}0.007 \\
(0.21)\end{array}$ \\
\hline Female & & & $\begin{array}{c}-0.096^{* * *} \\
(-3.32)\end{array}$ & $\begin{array}{c}-0.105^{* * *} \\
(-3.44)\end{array}$ & $\begin{array}{c}-0.109 * * * \\
(-3.48)\end{array}$ \\
\hline Freshman & & & $\begin{array}{c}0.132^{* * *} \\
(4.27)\end{array}$ & $\begin{array}{c}0.115^{* * *} \\
(3.56)\end{array}$ & $\begin{array}{c}0.113^{* * *} \\
(3.30)\end{array}$ \\
\hline Altruist(-) (no) & & & & $\begin{array}{l}0.008 \\
(0.17)\end{array}$ & $\begin{array}{l}0.003 \\
(0.06)\end{array}$ \\
\hline Altruist $(-)$ (yes) & & & & $\begin{array}{l}-0.134 \\
(-1.86)\end{array}$ & $\begin{array}{c}-0.167^{*} \\
(-2.22)\end{array}$ \\
\hline Altruist(+) (no) & & & & $\begin{array}{c}-0.194^{* *} \\
(-2.79)\end{array}$ & $\begin{array}{c}-0.204^{* *} \\
(-2.83)\end{array}$ \\
\hline Altruist $(+)$ (yes) & & & & $\begin{array}{l}0.001 \\
(0.02)\end{array}$ & $\begin{array}{l}-0.003 \\
(-0.09)\end{array}$ \\
\hline Protest (no) & & & & & $\begin{array}{l}0.060 \\
(1.26)\end{array}$ \\
\hline Protest (yes) & & & & & $\begin{array}{c}0.126^{* * *} \\
(3.53) \\
\end{array}$ \\
\hline Pseudo $\mathrm{R}^{2}$ & 0.034 & 0.035 & 0.056 & 0.066 & 0.075 \\
\hline Log likelihood & -705.4 & -694.4 & -621.7 & -551.9 & -545.2 \\
\hline Observations & 1189 & 1174 & 1075 & 963 & 962 \\
\hline
\end{tabular}

Probit estimation; marginal effects for benchmark students; discrete changes from 0 to 1 for indicator variables; z-statistic in parentheses. ${ }^{*} p<0.05,{ }^{* *} p<0.01,{ }^{* * *} p<0.001$.

Unlike in the decision to vote in favor or against, visiting others does not have a significant effect on the decision to take part (column 2). Being female reduces the probability of voting, whereas being a freshman increases it (column 3). However, we cannot disentangle alternative possible explanations driving the freshman effect. It could be based on the fact that the savings of freshmen refer to a shorter period of time, or alternatively, it may reflect that many freshmen are more easily reached by voting advertisements or more enthusiastic about taking the opportunity to vote in this referendum. 
Columns (4) and (5) introduce variables measuring the joint effect of social preferences and expressive concerns. We find large negative effects for altruist(-) (yes) and altruist $(+)$ (no), the first effect being statistically significant in column (5) and the second one in both columns. The first of these variables equals one for an individual who states that he or she cares for the benefit of others, thinks that others on average lose out by the ticket, and voted or would have voted in favor of the ticket. According to column (5), such an individual is $17 \%$ less likely to participate than someone who does not mention that he or she cares for others. Similarly, an altruist $(+)$ (no), i.e. someone who mentions to care for others, thinks that other students gain from the ticket, and voted or would have voted against, is $20 \%$ less likely to take part. These two effects show that students who consider the benefits of others but whose intended vote goes against the perceived interests of others are substantially less likely to participate. In contrast, the variables altruist(-) (no) and altruist(+) (yes) are not significantly different from zero.

This result can be explained by Corollary 2. To see this, consider a group of voters who have zero or very small net cost of participation $c_{i}-d_{i}$. Among these voters, everyone who has no social or expressive concern for others will participate and vote in line with his or her monetary interests. Those whose social or expressive concerns for others go in the same direction as their pocketbook motive will also participate. Thus, adding an altruistic motive which is in line with monetary interests does not change the participation decision if net costs of participation are very small. This explains why the variables altruist(-) (no) and altruist(+) (yes) have no impact. In contrast, voters whose expressive concern for others suggests voting against their monetary interests face a trade-off. As Corollary 2 shows, they may solve this conflict by abstaining. This explains the negative impacts of altruist(-) (yes) and altruist(+) (no).

Those who mention protest motives took part in the referendum with higher probability (column 5), even if the corresponding variable is significant only for those whose actual or intended vote is yes. Possibly, students who mention protest motives are generally more engaged in the debate about the ticket, and therefore more likely to turn out. They may also want to support student representatives, who campaigned for accepting the ticket, and therefore felt compelled to vote in favor, even if they still considered the price too high. Altogether, we see this result in line with Proposition 2(ii), which states that stronger expressive or social concerns make participation more attractive.

Our results on the participation decision imply that a substantial share of the electorate considered their probability of being pivotal to be non-negligible. To see this, note first that if the net cost of participation $c_{i}-d_{i}$ were non-positive, then every individual whose monetary and social or expressive benefits both are in favor 
of a yes vote would participate by Equation (2). In our data, however, among those who at the same time have positive stakes and are altruist $(+)($ yes $), 24 \%$ did not vote. Hence, for a substantial share of voters the net cost of voting is positive. The fact that we find a positive and statistically significant coefficient on positive monetary stakes therefore implies that the perceived probability $p_{i}^{1}$ is non-negligible for a substantial share of the electorate.

We next examine whether students with high gains from the ticket drive the finding that pocketbook benefits are relevant for participation. For this purpose, we gradually remove observations with the highest net savings from the dataset. Table 14 contains corresponding average marginal effects for benchmark students. In regression (1), we omit the top $5 \%$ in terms of net savings, whereas in regression (6), we omit the top $30 \%$. Positive stakes have a positive and highly significant effect if we use almost all of the observations. The more observations with high net savings we remove, the smaller the size and z-statistic of the marginal effects become. Looking at the bottom $75 \%$ only, the effect is no longer significantly different from zero. In contrast, the significance of most other variables suffers much less from this reduction of the sample, and the marginal effects are much more stable. This suggests that the loss of significance for the stakes variable should not be attributed to the smaller sample size alone. Instead, it appears that in particular students with very high gains from the ticket are responsible for the effect found for the full sample in Table 13, as they made sure to take part in the referendum. This is again in line with Proposition 2(i), which requires that, to induce participation, monetary stakes must exceed a certain threshold determined by social and expressive concerns as well as the net cost of participation.

Some econometric issues may arise related to the estimation of the participation equation. The indicator for the actual or intended vote might not be exogenous. This would be the case if the error terms in the voting and the participation equations (6) and (8) were correlated, for example if there is an omitted variable which affects both decisions. However, it is not clear what such an omitted variable could be and in which direction it would bias our results.

Reverse causality should not be a problem here. The variables capturing travel patterns and demographics are clearly not affected by the decision to vote. The protest variable is derived from the write-in section; consequently, it appears unlikely that it captures ex-post rationalization of the participation decision. In contrast, stated altruism could be affected by the participation decision. One could argue that it is easy to claim noble motivations if one does not make an actual decision. In this case, we would expect the share of those who state that they care for others and would have voted in line with the perceived interest of others to be higher among non-voters than among voters. This would imply negative coefficients of the 
Table 14: Taking part, reduced sample - Dataset I

\begin{tabular}{|c|c|c|c|c|c|c|}
\hline \multicolumn{7}{|c|}{ Dependent Variable: Taking Part in Referendum } \\
\hline & $(1)$ & $(2)$ & $(3)$ & $(4)$ & $(5)$ & $(6)$ \\
\hline Bottom & $95 \%$ & $90 \%$ & $85 \%$ & $80 \%$ & $75 \%$ & $70 \%$ \\
\hline \multirow[t]{2}{*}{ Log pos. stakes } & $0.038^{* * *}$ & $0.035^{* *}$ & $0.036^{* *}$ & $0.030^{*}$ & 0.019 & 0.007 \\
\hline & $(3.48)$ & $(2.86)$ & $(2.79)$ & $(2.01)$ & $(1.04)$ & $(0.34)$ \\
\hline \multirow[t]{2}{*}{ Neg. stakes } & 0.0015 & 0.0012 & 0.0013 & 0.0010 & 0.0006 & 0.0003 \\
\hline & $(1.54)$ & $(1.24)$ & $(1.29)$ & $(0.95)$ & $(0.57)$ & $(0.27)$ \\
\hline \multirow[t]{2}{*}{ Leisure/work } & 0.040 & 0.037 & 0.032 & 0.040 & 0.027 & -0.001 \\
\hline & $(0.66)$ & $(0.58)$ & $(0.48)$ & $(0.57)$ & $(0.37)$ & $(-0.02)$ \\
\hline \multirow{2}{*}{ Visiting others } & 0.009 & 0.010 & 0.016 & 0.018 & 0.002 & -0.013 \\
\hline & $(0.28)$ & $(0.30)$ & $(0.45)$ & $(0.47)$ & $(0.04)$ & $(-0.33)$ \\
\hline \multirow[t]{2}{*}{ Female } & $-0.109 * * *$ & $-0.112 * * *$ & $-0.111^{* *}$ & $-0.104^{* *}$ & $-0.110^{* *}$ & $-0.099^{*}$ \\
\hline & $(-3.34)$ & $(-3.29)$ & $(-3.18)$ & $(-2.87)$ & $(-2.91)$ & $(-2.53)$ \\
\hline \multirow[t]{2}{*}{ Freshman } & $0.119^{* *}$ & $0.122^{* *}$ & $0.117^{* *}$ & $0.126^{* *}$ & $0.127^{* *}$ & $0.130 * *$ \\
\hline & $(3.22)$ & $(3.14)$ & $(2.89)$ & $(2.91)$ & $(2.72)$ & $(2.63)$ \\
\hline \multirow{2}{*}{$\operatorname{Altruist}(-)($ no) } & 0.006 & 0.007 & 0.006 & 0.000 & 0.001 & 0.005 \\
\hline & $(0.11)$ & $(0.13)$ & $(0.10)$ & $(0.01)$ & $(0.02)$ & $(0.08)$ \\
\hline \multirow{2}{*}{$\operatorname{Altruist}(-)$ (yes) } & -0.111 & -0.107 & -0.148 & -0.144 & -0.151 & -0.123 \\
\hline & $(-1.38)$ & $(-1.30)$ & $(-1.70)$ & $(-1.56)$ & $(-1.54)$ & $(-1.16)$ \\
\hline \multirow{2}{*}{ Altruist $(+)$ (no) } & $-0.206^{* *}$ & $-0.208^{* *}$ & $-0.212^{* *}$ & $-0.216^{* *}$ & $-0.220 * *$ & $-0.215^{* *}$ \\
\hline & $(-2.83)$ & $(-2.83)$ & $(-2.88)$ & $(-2.92)$ & $(-2.92)$ & $(-2.84)$ \\
\hline \multirow{2}{*}{ Altruist $(+)$ (yes) } & -0.010 & -0.008 & -0.015 & -0.022 & 0.000 & 0.030 \\
\hline & $(-0.26)$ & $(-0.21)$ & $(-0.36)$ & $(-0.50)$ & $(0.00)$ & $(0.64)$ \\
\hline \multirow[t]{2}{*}{ Protest (no) } & 0.063 & 0.066 & 0.061 & 0.066 & 0.080 & 0.096 \\
\hline & $(1.28)$ & (1.31) & (1.18) & $(1.23)$ & $(1.47)$ & $(1.74)$ \\
\hline \multirow[t]{2}{*}{ Protest (yes) } & $0.140 * * *$ & $0.145 * * *$ & $0.146 * * *$ & $0.150 * * *$ & $0.190 * * *$ & $0.183^{* * *}$ \\
\hline & $(3.70)$ & $(3.69)$ & $(3.51)$ & $(3.37)$ & $(4.30)$ & $(3.72)$ \\
\hline Pseudo $\mathrm{R}^{2}$ & 0.071 & 0.062 & 0.059 & 0.050 & 0.050 & 0.042 \\
\hline Log likelihood & -520.0 & -508.2 & -487.1 & -469.2 & -444.3 & -422.8 \\
\hline Observations & 902 & 862 & 817 & 769 & 719 & 673 \\
\hline
\end{tabular}

variables altruist(-) (no) and altruist(+) (yes). Similarly, we would expect that among those who did not vote a lower share would admit that they would have voted against the interest of others than among those that actually voted. This would lead to positive coefficients of the variables altruist(-) (yes) and altruist $(+)$ (no). These predictions are opposite to the observed patterns in Table 13, which speaks against this kind of reverse causality.

In summary, the results from this section shed some light on the motives for voting in referenda. First, our results are in line with the theory of instrumental voting, which predicts that for a given probability of being pivotal and a given cost of voting, participation should increase with stakes. More specifically, we conclude that those who gain substantially, and hence lose substantially if the ticket fails, particularly drive this result. Thus, in a referendum, one may expect disproportion- 
ately higher turnout by voters who stand to benefit or lose substantially when the proposal passes, whereas voters who are affected only moderately are more likely to abstain. Second, we also find evidence that social preferences or expressive considerations, for example protest motives or a concern for others, have an impact on participation.

\section{Conclusion}

We provide a theory of individual voting and turnout decisions which integrates monetary benefits, social preferences, and expressive concerns. Social preferences are instrumental in the sense that voters want to change the outcome in a way that benefits others. The theory of expressive voting suggests that voters perceive their probability of being pivotal to be negligible, and that they derive utility from voting in a particular way irrespective of the outcome. A major challenge in previous literature on voting has been how to distinguish social preferences from expressive motivations, as both could be driven by what appears good for others. Our model allows to identify conditions under which each of these motives can be shown to be relevant.

We show that pocketbook benefits being relevant indicates that either voters do not perceive their impact on the outcome to be negligible, or expressive motivations do not play a role in the decision on how to vote. Conversely, if own pocketbook benefits do not explain voting, then voting is expressive. If the perceived probability of being pivotal is non-negligible, social preferences and expressive concerns are observationally equivalent. In terms of whether to vote, we show that if pocketbook motives and expressive concerns would require voting differently, abstention can be rational even if voting is costless. This would be the case if expressive feelings from abstention are the same as expressive feelings from voting differently than one would have actually done. Abstaining is then a cheaper way of obtaining the expressive benefit than voting against one's pocketbook.

We test our theory using individual data on referenda on deeply discounted flat rate tickets for train, bus, and cultural services held among university students. The service becomes much cheaper by being bought collectively; however, all members of society, including those who do not use the service, must pay to finance it. The same logic applies to any collective provision of a public good.

Our results show that monetary interests are a major driver of both turnout and voting decisions. However, we also find that in addition to this 'pocketbook voting', social or expressive motives such as the costs and benefits to other students or the desire to support local public transportation or cultural life are also important and occasionally even decisive for the referendum outcome. Based on our 
theory, the strong empirical support of pocketbook voting rules out a purely expressive explanation of observed voting behavior. The fact that also the probability of participation increases in monetary stakes implies that a substantial share of the electorate considered their probability of affecting the outcome to be non-negligible.

Our setting can be described as a real-world laboratory of direct democracy. Like in a laboratory, voters faced a clearly defined decision and had very good information on the individual costs and benefits associated with both outcomes. Thus, confounding influences such as asymmetric information should be minimized. At the same time, the polity to which the respondents belong is real and the social ties between the participants are independent of researchers. Since the decisions we study have strong parallels with decisions on local public goods, our results are particularly informative for direct democracy at the local level. We invite other researchers to test the predictions of our theory in other settings. It would be especially interesting to compare the relative importance of pocketbook benefits, social preferences, and expressive motives across referenda taking place at different levels of government. Jurisdictions like California and Switzerland would be fertile testing grounds for such analyses.

\section{Appendix}

\section{A.I Data handling}

The survey for Dataset I was conducted online between July and November 2010. It was advertised in lectures, on posters on campus, and in two e-mails that were sent to all students of University of Goettingen from the office of student affairs. On the survey webpage students were informed that the survey was conducted for research purposes and about a possibility to participate in a lottery. At the end of the questionnaire, students were provided with a link to another webpage, where they could register for the lottery. In order to identify winners without ambiguity, we required the (unique) student ID number for a registration for the lottery as well as an e-mail address. For the sake of data protection, questionnaire data and lottery data were collected in separate databases. As the savings variable plays a key role in explaining the decision on whether to vote and if voting, on how to vote, we excluded observations without information on voting behavior or for which we cannot assess savings, because, for instance, we lack data on traveling behavior or the corresponding student started studying only one month before the referendum took place. Besides, we removed less than fifteen observations from the dataset due to clear data errors or where answers given seemed highly implausible, such as visiting parents 20,000 times in one year. 
A challenge in the data management is that 75 ID numbers show up twice and on different days, suggesting that 75 students also show up twice in the dataset. Using the time stamps of the lottery data showed that in most cases, the responses were entered shortly after receiving an e-mail from the office of student affairs that was advertising the survey. Therefore, it is likely that these students had forgot that they had already answered a survey or thought that they should answer for a second time. After we explored this issue, we realized that we also have time stamps for survey responses, although in a separate data base. To guarantee the anonymity of respondents, we recruited a research assistant who was not otherwise connected to the project to select those entries from the lottery data that belong to duplicates in the lottery data base and to provide the two time stamps for each of the 75 pairs. As only time stamps were extracted pairwisely from the lottery database, anonymity was guaranteed at all times. Specifically, no individual information that could be used to identify the person behind a time stamp, such as a student ID number, was extracted from the lottery data.

Time stamps from the lottery were then assigned to the time stamps in the response dataset. For all pairs of time stamps, we examined responses that were submitted close to the time stamp in the lottery. For all pairs of these subsamples, we identified potential pairs of observations present in both subsamples, based on the data provided. Initial selection was made using gender, year of birth and the zip code of their parents. If students indicated that their parents do not live together, then the maternal, or if missing the paternal, zip code was used. If these three variables, year of birth, gender and parental zip code, were the same for observations in both subsamples, they were considered potential duplicates based on the data provided. Potential duplicates were then compared based on additional variables, such as subjects studied, travel frequencies, general attitudes and voting decisions, party preferences and reasons to be in favor and against the ticket. This procedure allowed us to identify 46 pairs of assigned duplicates. Consequently, we dropped the later entry of every pair of duplicates from the dataset. This left us with 29 pairs of duplicates which we could not identify. This corresponds to about two percent of the dataset and should, therefore, have only a tiny effect on the results. To be on the safe side, we also replicated the analysis of the second dataset without excluding observations based on this assignment. The results remain virtually unchanged.

\section{A.II Construction of the savings variable}

In the survey, students were asked about their parents' address (zip code) and how many times they visited their parents' residence within the last 12 months (July 1, 2009 - June 30, 2010) using the Bahn ticket. If students indicated that their parents 
did not live in the same city, questions were asked for both parents separately.

To translate trips to parents into monetary savings, the nearest train station covered by the Bahn ticket was identified for every parental address (zip code) using a standard route planner. ${ }^{18}$ Afterwards, for each station, the relevant price was derived. Therefore, we identified the suggested route to Goettingen using local trains for all stations on the Bahn tracks (dashed blue lines on the map, Figure 1) using software provided by Deutsche Bahn. At the time of the referendum, it was already known that students could use the MetroCan tracks (solid red lines) without additional costs. Consequently, savings per trip from the Bahn ticket are the price that would have to be paid to the station on this route where the "free" train (red) is entered. ${ }^{19}$

If the determined price was greater than 21 euros it was capped to this amount to reflect the possibility to buy the so called Lower-Saxony-ticket that is valid on all local trains in the state on the day of validation at this price. As furthermore every visit consists of the way back and forth, final savings are calculated as the product of the relevant price and twice the number of visits using the Bahn ticket. To also reflect the opportunity to buy a train ticket for all trains in Germany including high speed trains (BahnCard100) at a price of 3800 euros per year at the time of the survey, the savings variable is capped at 3800 euros.

For some students in Dataset I, Goettingen is not the nearest train station. Most of these students live in the same town as their parents, presumably with their parents. For these students, the savings variable captures direct monetary savings when they come to campus. If they live apart from their parents we calculate savings correspondingly, starting from their respective nearest station. Ten students, however, live outside Goettingen apart from their parents and save little on trips to them, but could primarily use this ticket for commuting. We refrain from calculating savings in these cases as they might severely misrepresent the benefit from the ticket. Consequently, these observations are dropped.

\section{A.III Approximation of $b_{i}^{1}-b_{i}^{0}$}

In this Appendix, we show how our measure of the monetary gains given in (7) relates to the equivalent variation $b_{i}^{1}-b_{i}^{0}$. Consider first voters with $h_{i}^{1}=0$, who do not care for the public good. These voters will also choose $h_{i}^{0}=0$, so that one immediately has $b_{i}^{1}-b_{i}^{0}=-t=\tau h_{i}^{1}-t$. Thus, for voters with a corner solution for the use of the public good, our measure is exact.

\footnotetext{
${ }^{18}$ Google maps, standard proposal for cars.

${ }^{19}$ Due to the non-linear pricing in the German railway market, this price is in most cases not equal to the price from a station to Goettingen less the price from the station where the free train is entered to Goettingen.
} 
Consider then voters with $h_{i}^{1}>0$. From the strict concavity of $w_{i}$ and the optimality conditions, for these individuals it follows $\tau \geq w_{i}^{\prime}\left(h_{i}^{0}\right)>0=w_{i}^{\prime}\left(h_{i}^{1}\right)$ and $0 \leq h_{i}^{0}<h_{i}^{1}$. Hence

$$
\begin{aligned}
w_{i}\left(h_{i}^{0}\right)+\tau\left(h_{i}^{1}-h_{i}^{0}\right) & \geq w_{i}\left(h_{i}^{0}\right)+w_{i}^{\prime}\left(h_{i}^{0}\right)\left(h_{i}^{1}-h_{i}^{0}\right)>w_{i}\left(h_{i}^{1}\right) \\
\Longrightarrow \tau h_{i}^{1}-t & >w_{i}\left(h_{i}^{1}\right)-t-\left[w_{i}\left(h_{i}^{0}\right)-\tau h_{i}^{0}\right]=b_{i}^{1}-b_{i}^{0} .
\end{aligned}
$$

There are three cases.

(a) $b_{i}^{1}-b_{i}^{0}>0$. Then with (A.9) also $\tau h_{i}^{1}-t>0$, and hence (A.9) shows that observed net savings $\tau h_{i}^{1}-t$ overestimate the true net monetary gain $b_{i}^{1}-b_{i}^{0}$.

(b) $\tau h_{i}^{1}-t<0$. Then with (A.9) also $b_{i}^{1}-b_{i}^{0}<0$, and hence, (A.9) is equivalent to $\left|\tau h_{i}^{1}-t\right|<\left|b_{i}^{1}-b_{i}^{0}\right|$. Hence, in this case the absolute value of observed net savings, or 'stakes', underestimates the absolute value of the true monetary loss.

(c) $b_{i}^{1}-b_{i}^{0}<0<\tau h_{i}^{1}-t$. Here, the approximation of the equivalent variation by net savings goes in the wrong direction. We expect, however, that the error introduced by this approximation is moderate given that this case occurs when net savings are rather close to zero.

To correct for the overestimation in case (a), positive net savings should be reduced. This is particularly important for observations with very high values of $h_{i}^{1}$, of which our dataset contains several. We choose to take the log to achieve this, after augmenting net savings by one to make sure the measure is positive also for observations with net savings between 0 and 1 .

To correct for the underestimation in case (b), we could in the same way enhance the loss $\left|\tau h_{i}^{1}-t\right|$ conferred by the public good. However, the difference between $h_{i}^{1}$ and $h_{i}^{0}$, and hence the difference between $\left|b_{i}^{1}-b_{i}^{0}\right|$ and $\left|\tau h_{i}^{1}-t\right|$ is rather small when net savings are negative. As mentioned above, the underestimation even vanishes completely for individuals who do not use the public good at all. Given that in our dataset a large share of the voters with negative net savings display such a corner solution, we consider it the best choice to approximate, for individuals with negative net savings, the loss conferred by the public good by the absolute value of net savings without any correction.

Altogether, we approximate

$$
b_{i}^{1}-b_{i}^{0}=\left\{\begin{array}{cl}
\ln \left(\tau h_{i}^{1}-t+1\right) & \text { if } \tau h_{i}^{1}-t \geq 0 \\
\tau h_{i}^{1}-t & \text { if } \tau h_{i}^{1}-t<0 .
\end{array}\right.
$$

Notice that this function is differentiable at $\tau h_{i}^{1}-t=0$. At this point, on both branches, it takes on the value 0 and the first derivative is 1 . 


\section{A.IV Coefficients}

Table A.1: Bahn ticket - Dataset I, coefficients for Table 9

\begin{tabular}{|c|c|c|c|c|c|}
\hline \multicolumn{6}{|c|}{ Dependent Variable: Supporting Bahn Ticket } \\
\hline & (1) & $(2)$ & $(3)$ & $(4)$ & $(5)$ \\
\hline Log pos. net savings & $\begin{array}{c}0.200^{* * *} \\
(0.04)\end{array}$ & $\begin{array}{c}0.231^{* * *} \\
(0.05)\end{array}$ & $\begin{array}{c}0.232^{* * *} \\
(0.05)\end{array}$ & $\begin{array}{c}0.203^{* * *} \\
(0.06)\end{array}$ & $\begin{array}{c}0.200^{* * *} \\
(0.06)\end{array}$ \\
\hline Neg. net savings & $\begin{array}{l}0.006 \\
(0.00)\end{array}$ & $\begin{array}{l}0.001 \\
(0.00)\end{array}$ & $\begin{array}{l}0.002 \\
(0.00)\end{array}$ & $\begin{array}{l}0.006 \\
(0.00)\end{array}$ & $\begin{array}{l}0.006 \\
(0.00)\end{array}$ \\
\hline Leisure/work & & $\begin{array}{l}0.235 \\
(0.24)\end{array}$ & $\begin{array}{l}0.101 \\
(0.26)\end{array}$ & $\begin{array}{l}0.108 \\
(0.28)\end{array}$ & $\begin{array}{l}0.123 \\
(0.28)\end{array}$ \\
\hline Visiting others & & $\begin{array}{c}1.053^{* * *} \\
(0.11)\end{array}$ & $\begin{array}{c}1.095^{* * *} \\
(0.12)\end{array}$ & $\begin{array}{c}1.001^{* * *} \\
(0.14)\end{array}$ & $\begin{array}{c}1.023^{* * *} * \\
(0.14)\end{array}$ \\
\hline Female & & & $\begin{array}{l}0.183 \\
(0.12)\end{array}$ & $\begin{array}{l}0.131 \\
(0.13)\end{array}$ & $\begin{array}{l}0.126 \\
(0.13)\end{array}$ \\
\hline Freshman & & & $\begin{array}{l}0.260 \\
(0.16)\end{array}$ & $\begin{array}{c}0.373^{*} \\
(0.19)\end{array}$ & $\begin{array}{l}0.405^{*} \\
(0.19)\end{array}$ \\
\hline Altruist $(-)$ & & & & $\begin{array}{c}-0.534^{* *} \\
(0.18)\end{array}$ & $\begin{array}{c}-0.521^{* *} \\
(0.18)\end{array}$ \\
\hline Altruist $(+)$ & & & & $\begin{array}{c}1.016^{* * *} \\
(0.16)\end{array}$ & $\begin{array}{c}1.022^{* * * *} \\
(0.17)\end{array}$ \\
\hline Protest & & & & & $\begin{array}{r}-0.290 \\
(0.15)\end{array}$ \\
\hline Constant & $\begin{array}{c}0.312 \\
(0.22) \\
\end{array}$ & $\begin{array}{c}-0.640^{*} \\
(0.25)\end{array}$ & $\begin{array}{c}-0.681^{*} \\
(0.28)\end{array}$ & $\begin{array}{l}-0.564 \\
(0.33)\end{array}$ & $\begin{array}{l}-0.474 \\
(0.34)\end{array}$ \\
\hline Pseudo $\mathrm{R}^{2}$ & 0.232 & 0.321 & 0.344 & 0.438 & 0.442 \\
\hline Log likelihood & -392.2 & -344.4 & -302.2 & -234.5 & -232.6 \\
\hline Observations & 818 & 810 & 741 & 669 & 669 \\
\hline
\end{tabular}


Table A.2: Train ticket - Dataset II, coefficients for Table 10

\begin{tabular}{lcccc}
\hline \hline \multicolumn{4}{c}{ Dependent Variable: Supporting Train Ticket } \\
\hline & $(1)$ & $(2)$ & $(3)$ & $(4)$ \\
\hline Rarely $(\leq 5 /$ year $)$ & $1.131^{* * *}$ & $1.025^{* * *}$ & $1.099^{* * *}$ & $1.060^{* * *}$ \\
& $(0.17)$ & $(0.18)$ & $(0.19)$ & $(0.20)$ \\
Sometimes (monthly) & $2.047^{* * *}$ & $1.980^{* * *}$ & $1.997^{* * *}$ & $1.976^{* * *}$ \\
& $(0.18)$ & $(0.18)$ & $(0.20)$ & $(0.21)$ \\
Often (weekly) & $2.452^{* * *}$ & $2.392^{* * *}$ & $2.386^{* * *}$ & $2.334^{* * *}$ \\
& $(0.21)$ & $(0.21)$ & $(0.23)$ & $(0.24)$ \\
Very often $(\geq 2 /$ week) & $2.574^{* * *}$ & $2.535^{* * *}$ & $2.528^{* * *}$ & $2.439^{* * *}$ \\
& $(0.21)$ & $(0.22)$ & $(0.24)$ & $(0.24)$ \\
Female & & $0.334^{* *}$ & $0.301^{* *}$ & $0.260^{*}$ \\
& & $(0.11)$ & $(0.12)$ & $(0.12)$ \\
Freshman & & $-0.251^{*}$ & -0.179 & -0.122 \\
& & $(0.11)$ & $(0.12)$ & $(0.13)$ \\
Savings to others & & & $0.826^{* * *}$ & $0.613^{* * *}$ \\
& & & $(0.13)$ & $(0.15)$ \\
Environment & & & & $0.730^{* * *}$ \\
& & & & \\
Constant & $-0.628^{* * *}$ & $-0.635^{* * *}$ & $-0.912^{* * *}$ & $-0.947^{* * *}$ \\
& $(0.15)$ & $(0.17)$ & $(0.19)$ & $(0.20)$ \\
\hline Pseudo $\mathrm{R}^{2}$ & 0.285 & 0.297 & 0.342 & 0.364 \\
Log likelihood & -356.2 & -334.4 & -298.1 & -277.9 \\
Observations & 1247 & 1191 & 1163 & 1145 \\
\hline \hline
\end{tabular}


Table A.3: Bus ticket - Dataset II, coefficients for Table 11

\begin{tabular}{|c|c|c|c|c|}
\hline \multicolumn{5}{|c|}{ Dependent Variable: Supporting Bus Ticket } \\
\hline & (1) & $(2)$ & $(3)$ & $(4)$ \\
\hline Rarely ( 1 or $2 /$ semester) & $\begin{array}{c}0.659^{* * *} \\
(0.10)\end{array}$ & $\begin{array}{c}0.672^{* * *} \\
(0.10)\end{array}$ & $\begin{array}{c}0.654^{* * *} \\
(0.10)\end{array}$ & $\begin{array}{c}0.631^{* * *} \\
(0.11)\end{array}$ \\
\hline Sometimes (monthly) & $\begin{array}{c}1.106^{* * *} \\
(0.12)\end{array}$ & $\begin{array}{c}1.188^{* * *} \\
(0.13)\end{array}$ & $\begin{array}{c}1.159^{* * * *} \\
(0.13)\end{array}$ & $\begin{array}{c}1.160^{* * *} \\
(0.14)\end{array}$ \\
\hline Often (weekly) & $\begin{array}{c}1.690^{* * *} * \\
(0.16)\end{array}$ & $\begin{array}{c}1.735^{* * *} \\
(0.16)\end{array}$ & $\begin{array}{c}1.746^{* * *} \\
(0.16)\end{array}$ & $\begin{array}{c}1.653^{* * *} \\
(0.17)\end{array}$ \\
\hline Very often $(\geq 2 /$ week $)$ & $\begin{array}{c}2.306^{* * *} \\
(0.16)\end{array}$ & $\begin{array}{c}2.339^{* * *} \\
(0.17)\end{array}$ & $\begin{array}{c}2.350^{* * *} \\
(0.18)\end{array}$ & $\begin{array}{c}2.222^{* * *} \\
(0.19)\end{array}$ \\
\hline Female & & $\begin{array}{r}-0.003 \\
(0.08)\end{array}$ & $\begin{array}{r}-0.023 \\
(0.09)\end{array}$ & $\begin{array}{l}0.026 \\
(0.09)\end{array}$ \\
\hline Freshman & & $\begin{array}{c}0.249^{* *} \\
(0.09)\end{array}$ & $\begin{array}{c}0.273^{* *} \\
(0.09)\end{array}$ & $\begin{array}{c}0.238^{*} \\
(0.09)\end{array}$ \\
\hline Savings to others & & & $\begin{array}{c}0.621^{* * *} \\
(0.10)\end{array}$ & $\begin{array}{c}0.509 * * * \\
(0.11)\end{array}$ \\
\hline Strengthening bus system & & & & $\begin{array}{c}1.179^{* * *} \\
(0.18)\end{array}$ \\
\hline Constant & $\begin{array}{c}-0.710^{* * *} \\
(0.06)\end{array}$ & $\begin{array}{c}-0.799^{* * * *} \\
(0.08)\end{array}$ & $\begin{array}{c}-0.915^{* * *} \\
(0.09)\end{array}$ & $\begin{array}{c}-0.959^{* * * *} \\
(0.09)\end{array}$ \\
\hline Pseudo $\mathrm{R}^{2}$ & 0.224 & 0.232 & 0.252 & 0.285 \\
\hline Log likelihood & -668.1 & -629.3 & -589.1 & -539.1 \\
\hline Observations & 1242 & 1183 & 1137 & 1090 \\
\hline
\end{tabular}


Table A.4: Culture ticket - Dataset II, coefficients for Table 12

\begin{tabular}{|c|c|c|c|c|}
\hline \multicolumn{5}{|c|}{ Dependent Variable: Supporting Culture Ticket } \\
\hline & (1) & $(2)$ & $(3)$ & (4) \\
\hline Rarely ( 1 or $2 /$ year) & $\begin{array}{c}0.982^{* * *} \\
(0.09)\end{array}$ & $\begin{array}{c}1.030^{* * *} \\
(0.10)\end{array}$ & $\begin{array}{c}0.961^{* * *} \\
(0.10)\end{array}$ & $\begin{array}{c}0.931^{* * *} \\
(0.12)\end{array}$ \\
\hline Sometimes ( 3 to $5 /$ year) & $\begin{array}{c}1.744^{* * *} \\
(0.15)\end{array}$ & $\begin{array}{c}1.758^{* * *} \\
(0.16)\end{array}$ & $\begin{array}{c}1.677^{* * *} \\
(0.17)\end{array}$ & $\begin{array}{c}1.664^{* * *} \\
(0.22)\end{array}$ \\
\hline Often (6 to $10 /$ year) & $\begin{array}{c}2.164^{* * *} \\
(0.33)\end{array}$ & $\begin{array}{c}2.198^{* * *} \\
(0.34)\end{array}$ & $\begin{array}{c}2.138^{* * *} * \\
(0.35)\end{array}$ & $\begin{array}{c}1.901^{* * *} \\
(0.44)\end{array}$ \\
\hline Very often $(>10 /$ year $)$ & $\begin{array}{c}1.915^{* * *} \\
(0.30)\end{array}$ & $\begin{array}{c}2.103 * * * \\
(0.35)\end{array}$ & $\begin{array}{c}1.855^{* * *} \\
(0.36)\end{array}$ & $\begin{array}{c}1.523^{* *} \\
(0.48)\end{array}$ \\
\hline Female & & $\begin{array}{c}0.217^{* *} \\
(0.08)\end{array}$ & $\begin{array}{l}0.220^{*} \\
(0.09)\end{array}$ & $\begin{array}{l}0.085 \\
(0.10)\end{array}$ \\
\hline Freshman & & $\begin{array}{c}0.292^{* *} \\
(0.10)\end{array}$ & $\begin{array}{c}0.327^{* *} \\
(0.10)\end{array}$ & $\begin{array}{c}0.338^{* *} \\
(0.12)\end{array}$ \\
\hline Savings to others & & & $\begin{array}{c}0.726^{* * * *} \\
(0.11)\end{array}$ & $\begin{array}{c}0.405^{* *} \\
(0.15)\end{array}$ \\
\hline Others should go & & & & $\begin{array}{c}1.293^{* * *} \\
(0.19)\end{array}$ \\
\hline Strengthening local culture & & & & $\begin{array}{c}1.344^{* * *} \\
(0.13)\end{array}$ \\
\hline Constant & $\begin{array}{c}-0.463^{* * *} \\
(0.05) \\
\end{array}$ & $\begin{array}{c}-0.681^{* * *} \\
(0.07) \\
\end{array}$ & $\begin{array}{c}-0.767^{* * *} \\
(0.08) \\
\end{array}$ & $\begin{array}{c}-1.141^{* * * *} \\
(0.10) \\
\end{array}$ \\
\hline Pseudo $\mathrm{R}^{2}$ & 0.192 & 0.191 & 0.215 & 0.440 \\
\hline Log likelihood & -664.7 & -633.7 & -571.1 & -378.7 \\
\hline Observations & 1189 & 1131 & 1055 & 988 \\
\hline
\end{tabular}


Table A.5: Taking part - Dataset I, coefficients for Table 13

\begin{tabular}{|c|c|c|c|c|c|}
\hline \multicolumn{6}{|c|}{ Dependent Variable: Taking Part in Referendum } \\
\hline & $(1)$ & $(2)$ & $(3)$ & $(4)$ & $(5)$ \\
\hline Log pos. stakes & $\begin{array}{c}0.104^{* * *} \\
(0.03)\end{array}$ & $\begin{array}{c}0.111^{* * * *} \\
(0.03)\end{array}$ & $\begin{array}{c}0.129^{* * *} \\
(0.03)\end{array}$ & $\begin{array}{c}0.139^{* * *} \\
(0.04)\end{array}$ & $\begin{array}{c}0.141^{* * *} \\
(0.04)\end{array}$ \\
\hline Neg. stakes & $\begin{array}{l}0.001 \\
(0.00)\end{array}$ & $\begin{array}{l}0.001 \\
(0.00)\end{array}$ & $\begin{array}{l}0.003 \\
(0.00)\end{array}$ & $\begin{array}{l}0.004 \\
(0.00)\end{array}$ & $\begin{array}{l}0.004 \\
(0.00)\end{array}$ \\
\hline Leisure/work & & $\begin{array}{r}-0.035 \\
(0.16)\end{array}$ & $\begin{array}{r}-0.008 \\
(0.18)\end{array}$ & $\begin{array}{l}0.118 \\
(0.19)\end{array}$ & $\begin{array}{l}0.113 \\
(0.19)\end{array}$ \\
\hline Visiting others & & $\begin{array}{l}0.019 \\
(0.09)\end{array}$ & $\begin{array}{l}0.018 \\
(0.09)\end{array}$ & $\begin{array}{l}0.032 \\
(0.10)\end{array}$ & $\begin{array}{l}0.021 \\
(0.10)\end{array}$ \\
\hline Female & & & $\begin{array}{l}-0.278^{* * *} \\
\quad(0.08)\end{array}$ & $\begin{array}{c}-0.310^{* * *} \\
\quad(0.09)\end{array}$ & $\begin{array}{c}-0.316^{* * *} \\
(0.09)\end{array}$ \\
\hline Freshman & & & $\begin{array}{c}0.487^{* * *} * \\
(0.13)\end{array}$ & $\begin{array}{c}0.434^{* *} \\
(0.14)\end{array}$ & $\begin{array}{c}0.408^{* *} \\
(0.14)\end{array}$ \\
\hline Altruist(-) (no) & & & & $\begin{array}{l}0.027 \\
(0.16)\end{array}$ & $\begin{array}{l}0.009 \\
(0.16)\end{array}$ \\
\hline Altruist (-) (yes) & & & & $\begin{array}{c}-0.390 * \\
(0.20)\end{array}$ & $\begin{array}{c}-0.471^{*} \\
(0.20)\end{array}$ \\
\hline Altruist $(+)$ (no) & & & & $\begin{array}{c}-0.549^{* *} \\
(0.18)\end{array}$ & $\begin{array}{c}-0.567^{* *} \\
(0.19)\end{array}$ \\
\hline Altruist $(+)$ (yes) & & & & $\begin{array}{c}0.002 \\
(0.11)\end{array}$ & $\begin{array}{r}-0.010 \\
(0.11)\end{array}$ \\
\hline Protest (no) & & & & & $\begin{array}{l}0.200 \\
(0.17)\end{array}$ \\
\hline Protest (yes) & & & & & $\begin{array}{c}0.469^{* *} \\
(0.15)\end{array}$ \\
\hline Constant & $\begin{array}{r}0.254 \\
(0.17) \\
\end{array}$ & $\begin{array}{l}0.212 \\
(0.19) \\
\end{array}$ & $\begin{array}{l}0.205 \\
(0.20) \\
\end{array}$ & $\begin{array}{r}0.174 \\
(0.22) \\
\end{array}$ & $\begin{array}{c}0.104 \\
(0.22)\end{array}$ \\
\hline Pseudo $\mathrm{R}^{2}$ & 0.034 & 0.035 & 0.056 & 0.066 & 0.075 \\
\hline Log likelihood & -705.4 & -694.4 & -621.7 & -551.9 & -545.2 \\
\hline Observations & 1189 & 1174 & 1075 & 963 & 962 \\
\hline
\end{tabular}


Table A.6: Taking part, reduced sample - Dataset I, coefficients for Table 14

\begin{tabular}{|c|c|c|c|c|c|c|}
\hline \multicolumn{7}{|c|}{ Dependent Variable: Taking Part in Referendum } \\
\hline & (1) & $(2)$ & (3) & (4) & $(5)$ & (6) \\
\hline Bottom & $95 \%$ & $90 \%$ & $85 \%$ & $80 \%$ & $75 \%$ & $70 \%$ \\
\hline \multirow[t]{2}{*}{ Log pos. stakes } & $0.139^{* * *}$ & $0.118^{* *}$ & $0.122^{* *}$ & 0.094 & 0.054 & 0.020 \\
\hline & $(0.04)$ & $(0.04)$ & $(0.04)$ & $(0.05)$ & $(0.05)$ & $(0.06)$ \\
\hline \multirow[t]{2}{*}{ Neg. stakes } & 0.004 & 0.003 & 0.004 & 0.003 & 0.002 & 0.001 \\
\hline & $(0.00)$ & $(0.00)$ & $(0.00)$ & $(0.00)$ & $(0.00)$ & $(0.00)$ \\
\hline \multirow[t]{2}{*}{ Leisure/work } & 0.126 & 0.113 & 0.095 & 0.115 & 0.077 & -0.003 \\
\hline & $(0.20)$ & $(0.20)$ & $(0.20)$ & $(0.21)$ & $(0.21)$ & $(0.22)$ \\
\hline \multirow[t]{2}{*}{ Visiting others } & 0.029 & 0.031 & 0.047 & 0.050 & 0.005 & -0.036 \\
\hline & $(0.10)$ & $(0.10)$ & $(0.10)$ & $(0.11)$ & $(0.11)$ & $(0.11)$ \\
\hline \multirow[t]{2}{*}{ Female } & $-0.309^{* * *}$ & $-0.309^{* *}$ & $-0.304^{* *}$ & $-0.279^{* *}$ & $-0.290^{* *}$ & $-0.259 *$ \\
\hline & $(0.09)$ & $(0.09)$ & $(0.10)$ & $(0.10)$ & $(0.10)$ & $(0.10)$ \\
\hline \multirow[t]{2}{*}{ Freshman } & $0.415^{* *}$ & $0.410^{* *}$ & $0.385^{* *}$ & $0.405^{* *}$ & $0.395^{*}$ & $0.396^{*}$ \\
\hline & $(0.14)$ & $(0.15)$ & $(0.15)$ & $(0.15)$ & $(0.16)$ & $(0.17)$ \\
\hline \multirow[t]{2}{*}{ Altruist $(-)$ (no) } & 0.017 & 0.021 & 0.016 & 0.001 & 0.004 & 0.013 \\
\hline & $(0.16)$ & $(0.16)$ & $(0.16)$ & $(0.17)$ & $(0.17)$ & $(0.17)$ \\
\hline \multirow[t]{2}{*}{ Altruist $(-)$ (yes) } & -0.314 & -0.297 & -0.402 & -0.383 & -0.394 & -0.319 \\
\hline & $(0.22)$ & $(0.22)$ & $(0.23)$ & $(0.24)$ & $(0.25)$ & $(0.27)$ \\
\hline \multirow[t]{2}{*}{ Altruist $(+)$ (no) } & $-0.561 * *$ & $-0.556^{* *}$ & $-0.563^{* *}$ & $-0.565^{* *}$ & $-0.567^{* *}$ & $-0.550^{* *}$ \\
\hline & $(0.19)$ & $(0.19)$ & $(0.19)$ & $(0.19)$ & $(0.19)$ & $(0.19)$ \\
\hline \multirow[t]{2}{*}{ Altruist $(+)$ (yes) } & -0.029 & -0.024 & -0.043 & -0.061 & 0.000 & 0.082 \\
\hline & $(0.11)$ & $(0.11)$ & $(0.12)$ & $(0.12)$ & $(0.13)$ & $(0.13)$ \\
\hline \multirow[t]{2}{*}{ Protest (no) } & 0.203 & 0.208 & 0.190 & 0.198 & 0.236 & 0.281 \\
\hline & $(0.17)$ & $(0.17)$ & $(0.17)$ & $(0.17)$ & $(0.17)$ & $(0.17)$ \\
\hline \multirow[t]{2}{*}{ Protest (yes) } & $0.505^{* *}$ & $0.508^{* *}$ & $0.505^{* *}$ & $0.498^{* *}$ & $0.649 * * *$ & $0.598^{* *}$ \\
\hline & $(0.16)$ & $(0.16)$ & $(0.17)$ & $(0.17)$ & $(0.19)$ & $(0.19)$ \\
\hline \multirow[t]{2}{*}{ Constant } & 0.101 & 0.158 & 0.146 & 0.206 & 0.295 & 0.348 \\
\hline & $(0.23)$ & $(0.24)$ & $(0.24)$ & $(0.25)$ & $(0.25)$ & $(0.26)$ \\
\hline Pseudo $\mathrm{R}^{2}$ & 0.071 & 0.062 & 0.059 & 0.050 & 0.050 & 0.042 \\
\hline Log likelihood & -520.0 & -508.2 & -487.1 & -469.2 & -444.3 & -422.8 \\
\hline Observations & 902 & 862 & 817 & 769 & 719 & 673 \\
\hline
\end{tabular}

Probit estimation; coefficients; standard errors in parentheses. Columns show percentiles with respect to net savings, e.g., column (1) contains those observations that belong to the bottom $95 \%$ with respect to net savings. ${ }^{*} p<0.05,{ }^{* *} p<0.01,{ }^{* * *} p<0.001$ 


\section{A.V Political parties and fields of study}

Table A.7: General political preferences - Dataset I, coefficients

\begin{tabular}{|c|c|}
\hline Dependent Variable & : Supporting Bahn Ticket \\
\hline & (1) \\
\hline Log pos. net savings & $\begin{array}{l}0.159^{*} \\
(0.07)\end{array}$ \\
\hline Neg. net savings & $\begin{array}{l}0.010^{*} \\
(0.00)\end{array}$ \\
\hline Leisure/work & $\begin{array}{l}0.331 \\
(0.34)\end{array}$ \\
\hline Visiting others & $\begin{array}{c}1.051^{* * * *} \\
(0.16)\end{array}$ \\
\hline Female & $\begin{array}{l}0.136 \\
(0.16)\end{array}$ \\
\hline Freshman & $\begin{array}{c}0.576^{* *} \\
(0.22)\end{array}$ \\
\hline Altruist(-) & $\begin{array}{c}-0.621^{* *} \\
(0.21)\end{array}$ \\
\hline Altruist(+) & $\begin{array}{c}1.118^{* * *} \\
(0.19)\end{array}$ \\
\hline Protest & $\begin{array}{c}-0.384^{*} \\
(0.17)\end{array}$ \\
\hline Social Democrats & $\begin{array}{l}-0.017 \\
(0.23)\end{array}$ \\
\hline Liberal Democrats & $\begin{array}{r}-0.270 \\
(0.28)\end{array}$ \\
\hline Green & $\begin{array}{l}-0.257 \\
(0.21)\end{array}$ \\
\hline Left & $\begin{array}{c}0.018 \\
(0.49)\end{array}$ \\
\hline Other parties & $\begin{array}{l}0.314 \\
(0.37)\end{array}$ \\
\hline Constant & $\begin{array}{c}-0.092 \\
(0.42) \\
\end{array}$ \\
\hline Pseudo $\mathrm{R}^{2}$ & 0.453 \\
\hline Log likelihood & -179.8 \\
\hline Observations & 534 \\
\hline
\end{tabular}


Table A.8: Fields of study - Dataset II, coefficients

\begin{tabular}{|c|c|c|c|}
\hline \multicolumn{4}{|c|}{ Dependent Variable: Supporting Ticket } \\
\hline & $\begin{array}{c}(1) \\
\text { Train } \\
\end{array}$ & $\begin{array}{l}(2) \\
\text { Bus }\end{array}$ & $\begin{array}{c}(3) \\
\text { Culture } \\
\end{array}$ \\
\hline Rarely $^{\diamond}$ & $\begin{array}{c}1.083^{* * *} \\
(0.20)\end{array}$ & $\begin{array}{c}0.640^{* * *} \\
(0.11)\end{array}$ & $\begin{array}{c}0.859^{* * *} \\
(0.13)\end{array}$ \\
\hline Sometimes $\diamond$ & $\begin{array}{l}2.010^{* * *} \\
(0.21)\end{array}$ & $\begin{array}{l}1.174 * * * \\
(0.14)\end{array}$ & $\begin{array}{c}1.642^{* * *} \\
(0.23)\end{array}$ \\
\hline Often $\diamond$ & $\begin{array}{l}2.402^{* * *} \\
\quad(0.24)\end{array}$ & $\begin{array}{c}1.644^{* * *} \\
(0.17)\end{array}$ & $\begin{array}{l}1.755^{* * *} \\
(0.45)\end{array}$ \\
\hline Very often $\diamond$ & $\begin{array}{l}2.438^{* * *} \\
(0.25)\end{array}$ & $\begin{array}{c}2.237^{* * *} \\
(0.19)\end{array}$ & $\begin{array}{c}1.430^{* *} \\
(0.51)\end{array}$ \\
\hline Savings to others & $\begin{array}{c}0.632^{* * *} \\
(0.15)\end{array}$ & $\begin{array}{c}0.500 * * * \\
(0.11)\end{array}$ & $\begin{array}{c}0.398^{* *} \\
(0.15)\end{array}$ \\
\hline Female & $\begin{array}{l}0.186 \\
(0.13)\end{array}$ & $\begin{array}{l}0.025 \\
(0.09)\end{array}$ & $\begin{array}{l}0.015 \\
(0.11)\end{array}$ \\
\hline Freshman & $\begin{array}{l}-0.117 \\
(0.13)\end{array}$ & $\begin{array}{l}0.242^{*} \\
(0.10)\end{array}$ & $\begin{array}{c}0.319^{*} \\
(0.13)\end{array}$ \\
\hline Environment & $\begin{array}{c}0.707^{* * *} \\
(0.18)\end{array}$ & & \\
\hline Strengthening bus system & & $\begin{array}{c}1.177^{* * *} \\
(0.18)\end{array}$ & \\
\hline Others should go & & & $\begin{array}{c}1.333^{* * *} \\
(0.19)\end{array}$ \\
\hline Strengthening local culture & & & $\begin{array}{c}1.317^{* * *} \\
(0.14)\end{array}$ \\
\hline Social sciences & $\begin{array}{c}0.149 \\
(0.17)\end{array}$ & $\begin{array}{c}0.086 \\
(0.12)\end{array}$ & $\begin{array}{c}0.560^{* * *} \\
(0.14)\end{array}$ \\
\hline Forestry/Agriculture & $\begin{array}{c}-0.623^{* *} \\
(0.22)\end{array}$ & $\begin{array}{l}0.262 \\
(0.19)\end{array}$ & $\begin{array}{r}-0.147 \\
(0.24)\end{array}$ \\
\hline Humanities & $\begin{array}{l}0.210 \\
(0.16)\end{array}$ & $\begin{array}{l}0.040 \\
(0.11)\end{array}$ & $\begin{array}{l}0.343^{*} \\
(0.14)\end{array}$ \\
\hline Geology/Geography & $\begin{array}{c}0.016 \\
(0.41)\end{array}$ & $\begin{array}{l}0.082 \\
(0.26)\end{array}$ & $\begin{array}{l}0.545 \\
(0.32)\end{array}$ \\
\hline Law & $\begin{array}{l}-0.046 \\
(0.20)\end{array}$ & $\begin{array}{l}0.318^{*} \\
(0.15)\end{array}$ & $\begin{array}{l}0.089 \\
(0.17)\end{array}$ \\
\hline Natural sciences & $\begin{array}{r}-0.191 \\
(0.21)\end{array}$ & $\begin{array}{l}-0.026 \\
(0.17)\end{array}$ & $\begin{array}{l}0.440^{*} \\
(0.20)\end{array}$ \\
\hline Other fields & $\begin{array}{l}0.397 \\
(0.47)\end{array}$ & $\begin{array}{l}-0.146 \\
(0.25)\end{array}$ & $\begin{array}{l}0.240 \\
(0.33)\end{array}$ \\
\hline Constant & $\begin{array}{c}-0.949 * * * \\
(0.21) \\
\end{array}$ & $\begin{array}{c}-1.039^{* * *} \\
(0.10)\end{array}$ & $\begin{array}{c}-1.336 * * * \\
(0.12)\end{array}$ \\
\hline Pseudo $\mathrm{R}^{2}$ & 0.381 & 0.290 & 0.460 \\
\hline Log Likelihood & -270.4 & -534.9 & -364.3 \\
\hline Observations & 1143 & 1088 & 986 \\
\hline
\end{tabular}

Probit estimation; coefficients; standard errors in parentheses.

$\diamond$ Definitions of the intensity of use differ: Rarely, Sometimes, Often, and Very often correspond to at most 5 times a year, monthly, weekly, and at least twice a week, respectively, for the train ticket; once or twice a semester, monthly, weekly, at least twice a week, respectively, during the lecture period for the bus ticket and once or twice a year, 3 to 5 times a year, 6 to 10 times a year, $>10$ times a year, respectively, for the culture ticket. The base categories for the fields of study is economic sciences. ${ }^{*} p<0.05,{ }^{* *} p<0.01,{ }^{* * *} p<0.001$. 
Table A.9: General political preferences - Dataset II, coefficients

\begin{tabular}{|c|c|c|c|}
\hline \multicolumn{4}{|c|}{ Dependent Variable: Supporting Ticket } \\
\hline & $\begin{array}{l}(1) \\
\text { Train }\end{array}$ & $\begin{array}{l}\text { (2) } \\
\text { Bus }\end{array}$ & $\begin{array}{c}(3) \\
\text { Culture }\end{array}$ \\
\hline Rarely & $\begin{array}{c}1.034^{* * *} \\
(0.21)\end{array}$ & $\begin{array}{c}0.585^{* * *} \\
(0.11)\end{array}$ & $\begin{array}{c}0.893^{* * *} \\
(0.14)\end{array}$ \\
\hline Sometimes $\diamond$ & $\begin{array}{c}1.930 * * * \\
(0.22)\end{array}$ & $\begin{array}{c}1.083^{* * *} \\
(0.15)\end{array}$ & $\begin{array}{c}1.712^{* * *} \\
(0.25)\end{array}$ \\
\hline Often $\diamond$ & $\begin{array}{c}2.408^{* * *} \\
(0.26)\end{array}$ & $\begin{array}{c}1.626^{* * *} \\
(0.18)\end{array}$ & $\begin{array}{c}1.804^{* * *} \\
(0.44)\end{array}$ \\
\hline Very often $\diamond$ & $\begin{array}{c}2.344 * * * \\
(0.25)\end{array}$ & $\begin{array}{c}2.104^{* * *} \\
(0.20)\end{array}$ & $\begin{array}{c}1.981^{* * * *} \\
(0.60)\end{array}$ \\
\hline Savings to others & $\begin{array}{c}0.690^{* * *} \\
(0.15)\end{array}$ & $\begin{array}{c}0.538^{* * *} \\
(0.12)\end{array}$ & $\begin{array}{c}0.366^{*} \\
(0.16)\end{array}$ \\
\hline Female & $\begin{array}{c}0.332^{*} \\
(0.13)\end{array}$ & $\begin{array}{c}0.040 \\
(0.10)\end{array}$ & $\begin{array}{l}-0.049 \\
(0.12)\end{array}$ \\
\hline Freshman & $\begin{array}{l}-0.176 \\
(0.14)\end{array}$ & $\begin{array}{c}0.272^{* *} \\
(0.10)\end{array}$ & $\begin{array}{l}0.346^{*} \\
(0.14)\end{array}$ \\
\hline Environment & $\begin{array}{c}0.663^{* * *} \\
(0.19)\end{array}$ & & \\
\hline Strengthening bus system & & $\begin{array}{c}1.225^{* * *} \\
(0.20)\end{array}$ & \\
\hline Others should go & & & $\begin{array}{c}1.267^{* * *} \\
(0.20)\end{array}$ \\
\hline Strengthening local culture & & & $\begin{array}{c}1.287^{* * *} \\
(0.15)\end{array}$ \\
\hline Social Democrats & $\begin{array}{l}-0.104 \\
(0.16)\end{array}$ & $\begin{array}{l}0.017 \\
(0.12)\end{array}$ & $\begin{array}{c}0.628^{* * * *} \\
(0.15)\end{array}$ \\
\hline Liberal Democrats & $\begin{array}{l}-0.562 \\
(0.29)\end{array}$ & $\begin{array}{l}-0.232 \\
(0.27)\end{array}$ & $\begin{array}{c}0.171 \\
(0.27)\end{array}$ \\
\hline Green & $\begin{array}{l}-0.104 \\
(0.17)\end{array}$ & $\begin{array}{l}-0.109 \\
(0.13)\end{array}$ & $\begin{array}{c}0.611^{* * *} \\
(0.16)\end{array}$ \\
\hline Left & $\begin{array}{l}-0.065 \\
(0.34)\end{array}$ & $\begin{array}{l}0.037 \\
(0.25)\end{array}$ & $\begin{array}{c}0.970^{* *} \\
(0.34)\end{array}$ \\
\hline Other parties & $\begin{array}{l}0.153 \\
(0.31)\end{array}$ & $\begin{array}{l}-0.076 \\
(0.23)\end{array}$ & $\begin{array}{l}0.260 \\
(0.28)\end{array}$ \\
\hline Constant & $\begin{array}{c}-0.884^{* * *} \\
(0.23)\end{array}$ & $\begin{array}{c}-0.935^{* * *} \\
(0.11)\end{array}$ & $\begin{array}{c}-1.486^{* * *} \\
(0.14)\end{array}$ \\
\hline Pseudo $\mathrm{R}^{2}$ & 0.371 & 0.281 & 0.466 \\
\hline Log Likelihood & -246.5 & -477.1 & -312.7 \\
\hline Observations & 1000 & 958 & 858 \\
\hline
\end{tabular}

Probit estimation; coefficients; standard errors in parentheses.

$\diamond$ Definitions of the intensity of use differ: Rarely, Sometimes, Often, and Very often correspond to at most 5 times a year, Often, and Very often correspond to at most 5 times a year, monthly, weekly, and at least twice a week, respectively, for the
train ticket; once or twice a semester, monthly, weekly, at least train ticket; once or twice a semester, monthly, weekly, at least twice a week, respectively, during the lecture period for the bus a year, $>10$ times a year, respectively, for the culture ticket. The base category for the party preferences is Christian Democrats. ${ }^{*} p<0.05,{ }^{* *} p<0.01,{ }^{* * *} p<0.001$ 
Table A.10: Political preferences and fields of study - Dataset II, coefficients

\begin{tabular}{|c|c|c|c|}
\hline \multicolumn{4}{|c|}{ Dependent Variable: Supporting Ticket } \\
\hline & $\begin{array}{c}\text { (1) } \\
\text { Train }\end{array}$ & $\begin{array}{l}\text { (2) } \\
\text { Bus }\end{array}$ & $\begin{array}{c}(3) \\
\text { Culture }\end{array}$ \\
\hline Rarely $\diamond$ & $\begin{array}{c}1.060^{* * *} \\
(0.22)\end{array}$ & $\begin{array}{c}0.583^{* * *} \\
(0.12)\end{array}$ & $\begin{array}{c}0.853^{* * *} \\
(0.14)\end{array}$ \\
\hline Sometimes $\diamond$ & $\begin{array}{c}1.963^{* * *} \\
(0.22)\end{array}$ & $\begin{array}{c}1.081 * * * \\
(0.15)\end{array}$ & $\begin{array}{c}1.728^{* * *} \\
(0.25)\end{array}$ \\
\hline Often $\diamond$ & $\begin{array}{c}2.475^{* * *} \\
(0.26)\end{array}$ & $\begin{array}{c}1.619^{* * *} \\
(0.18)\end{array}$ & $\begin{array}{c}1.762^{* * *} \\
(0.45)\end{array}$ \\
\hline Very often $\diamond$ & $\begin{array}{c}2.331^{* * *} \\
(0.26)\end{array}$ & $\begin{array}{c}2.112^{* * *} \\
(0.20)\end{array}$ & $\begin{array}{c}1.971 * * \\
(0.63)\end{array}$ \\
\hline Savings to others & $\begin{array}{c}0.718^{* * *} \\
(0.16)\end{array}$ & $\begin{array}{c}0.529^{* * *} \\
(0.12)\end{array}$ & $\begin{array}{l}0.361^{*} \\
(0.16)\end{array}$ \\
\hline Female & $\begin{array}{l}0.261 \\
(0.14)\end{array}$ & $\begin{array}{c}0.035 \\
(0.10)\end{array}$ & $\begin{array}{l}-0.062 \\
(0.12)\end{array}$ \\
\hline Freshman & $\begin{array}{l}-0.178 \\
(0.14)\end{array}$ & $\begin{array}{c}0.275^{* *} \\
(0.10)\end{array}$ & $\begin{array}{l}0.348^{*} \\
(0.14)\end{array}$ \\
\hline Environment & $\begin{array}{c}0.644^{* * *} \\
(0.19)\end{array}$ & & \\
\hline Strengthening bus system & & $\begin{array}{c}1.225^{* * *} \\
(0.20)\end{array}$ & \\
\hline Others should go & & & $\begin{array}{c}1.316^{* * * *} \\
(0.20)\end{array}$ \\
\hline Strengthening local culture & & & $\begin{array}{c}1.294^{* * *} \\
(0.15)\end{array}$ \\
\hline Social sciences & $\begin{array}{c}0.172 \\
(0.18)\end{array}$ & $\begin{array}{l}0.127 \\
(0.12)\end{array}$ & $\begin{array}{c}0.446^{* *} \\
(0.15)\end{array}$ \\
\hline Forestry/Agriculture & $\begin{array}{c}-0.661^{* *} \\
(0.24)\end{array}$ & $\begin{array}{l}0.165 \\
(0.21)\end{array}$ & $\begin{array}{r}-0.004 \\
(0.27)\end{array}$ \\
\hline Humanities & $\begin{array}{c}0.201 \\
(0.17)\end{array}$ & $\begin{array}{l}0.033 \\
(0.12)\end{array}$ & $\begin{array}{l}0.176 \\
(0.16)\end{array}$ \\
\hline Geology/Geography & $\begin{array}{l}0.013 \\
(0.42)\end{array}$ & $\begin{array}{l}0.267 \\
(0.28)\end{array}$ & $\begin{array}{l}0.608 \\
(0.36)\end{array}$ \\
\hline Law & $\begin{array}{l}-0.097 \\
(0.21)\end{array}$ & $\begin{array}{c}0.414^{*} \\
(0.16)\end{array}$ & $\begin{array}{l}0.073 \\
(0.18)\end{array}$ \\
\hline Natural sciences & $\begin{array}{r}-0.367 \\
(0.23)\end{array}$ & $\begin{array}{r}0.059 \\
(0.18)\end{array}$ & $\begin{array}{c}0.379 \\
(0.22)\end{array}$ \\
\hline Other fields & $\begin{array}{c}0.328 \\
(0.52)\end{array}$ & $\begin{array}{r}-0.065 \\
(0.28)\end{array}$ & $\begin{array}{c}0.231 \\
(0.38)\end{array}$ \\
\hline Social Democrats & $\begin{array}{l}-0.231 \\
(0.17)\end{array}$ & $\begin{array}{l}0.046 \\
(0.13)\end{array}$ & $\begin{array}{c}0.541^{* * *} \\
(0.16)\end{array}$ \\
\hline Liberal Democrats & $\begin{array}{c}-0.674^{*} \\
(0.29)\end{array}$ & $\begin{array}{r}-0.218 \\
(0.27)\end{array}$ & $\begin{array}{c}0.178 \\
(0.27)\end{array}$ \\
\hline Green & $\begin{array}{l}-0.221 \\
(0.19)\end{array}$ & $\begin{array}{l}-0.081 \\
(0.13)\end{array}$ & $\begin{array}{c}0.466^{* *} \\
(0.17)\end{array}$ \\
\hline Left & $\begin{array}{l}-0.217 \\
(0.35)\end{array}$ & $\begin{array}{l}0.058 \\
(0.26)\end{array}$ & $\begin{array}{c}0.930^{* *} \\
(0.36)\end{array}$ \\
\hline Other parties & $\begin{array}{l}0.106 \\
(0.33)\end{array}$ & $\begin{array}{l}-0.037 \\
(0.24)\end{array}$ & $\begin{array}{c}0.238 \\
(0.29)\end{array}$ \\
\hline Constant & $\begin{array}{c}-0.780^{* *} \\
(0.24)\end{array}$ & $\begin{array}{c}-1.057^{* * *} \\
(0.13)\end{array}$ & $\begin{array}{c}-1.612^{* * *} \\
(0.16)\end{array}$ \\
\hline Pseudo $\mathrm{R}^{2}$ & 0.391 & 0.287 & 0.477 \\
\hline Log likelihood & -238.6 & -472.9 & -306.0 \\
\hline Observations & 999 & 957 & 857 \\
\hline
\end{tabular}

Probit estimation; coefficients; standard errors in parentheses. $\diamond$ Definitions of the intensity of use differ: Rarely, Sometimes, Often, and Very often correspond to at most 5 times a year, monthly, weekly, and at least twice a week, respectively, for the train ticket; once or twice a semester, monthly, weekly, at least train ticket; once or twice a semester, monthly, weekly, at least
twice a week, respectively, during the lecture period for the bus twice a week, respectively, during the lecture period for the bus ticket and once or twice a year, 3 to 5 times a year, 6 to 10
times a year, $>10$ times a year, respectively, for the culture times a year, $>10$ times a year, respectively, for the culture
ticket. The base categories for the fields of study and party preferences are economic sciences and Christian Democrats, respectively. ${ }^{*} p<0.05,{ }^{* *} p<0.01,{ }^{* * *} p<0.001$ 


\section{References}

Akerlof, George A. and Rachel E. Kranton, "Economics and Identity," Quarterly Journal of Economics, 2000, 115 (3), 715-753.

Alesina, Alberto and George-Marios Angeletos, "Fairness and Redistribution," American Economic Review, 2005, 95 (4), 960-980.

Andersen, Jørgen J., Jon H. Fiva, and Gisle J. Natvik, "Voting when the Stakes are High," Journal of Public Economics, 2014, 110, 157-166.

Balafoutas, Loukas, Martin G. Kocher, Louis Putterman, and Matthias Sutter, "Equality, Equity and Incentives: An Experiment," European Economic Review, 2013, 60, 32-51.

Ben-Bassat, Avi and Momi Dahan, "Social Identity and Voting Behavior," Public Choice, 2012, 151 (1), 193-214.

Bénabou, Roland and Jean Tirole, "Belief in a Just World and Redistributive Politics," Quarterly Journal of Economics, 2006, 121 (2), 699-746.

Börgers, Tilman, "Costly Voting," American Economic Review, 2004, 94 (1), 5766.

Brennan, Geoffrey and James Buchanan, "Voter Choice: Evaluating Political Alternatives," American Behavioral Scientist, 1984, 28 (2), 185-201.

- and Alan Hamlin, "Expressive Voting and Electoral Equilibrium," Public Choice, 1998, 95 (1-2), 149-175.

California State Board of Equalization, "California Property Tax: An Overview," Publication 29, November 2012, available at http://www.boe.ca. gov/proptaxes/pdf/pub29.pdf (accessed September 28th, 2014) 2012.

Card, David, Christian Dustmann, and Ian Preston, "Immigration, Wages, and Compositional Amenities," Journal of the European Economic Association, 2012, $10(1), 78-119$.

Carlsson, Fredrik and Olof Johansson-Stenman, "Why Do You Vote and Vote as You Do?," Kyklos, 2010, 63 (4), 495-516.

Carter, John and Stephen D. Guerette, "An Experimental Study of Expressive Voting," Public Choice, 1992, 73, 251-260. 
Coate, Stephen and Michael Conlin, "A Group Rule: Utilitarian Approach to Voter Turnout: Theory and Evidence," American Economic Review, 2004, 94 (5), 1476-1504.

_ , _, and Andrea Moro, "The Performance of Pivotal-Voter Models in SmallScale Elections: Evidence from Texas Liquor Referenda," Journal of Public Economics, 2008, 92 (3-4), 582-596.

Coates, Dennis and Brad R. Humphreys, "Proximity Benefits and Voting on Stadium and Arena Subsidies," Journal of Urban Economics, 2006, 59 (2), 285299.

De Tocqueville, Alexis, De la démocratie en Amérique, Vol. I, Paris: GarnierFlammarion, 1981 [1835].

Deacon, Robert and Perry Shapiro, "Private Preference for Collective Goods Revealed through Voting on Referenda," American Economic Review, 1975, 65 (5), 943-955.

DellaVigna, Stefano and Ulrike Malmendier, "Paying not to Go to the Gym," American Economic Review, 2006, 96 (3), 694-719.

Dixit, Avinash and John Londregan, "The Determinants of Success of Special Interests in Redistributive Politics," Journal of Politics, 1996, 58 (4), 1132-1155.

Downs, Anthony, An Economic Theory of Democracy, New York: Harper and Row Publishers, 1957.

Duffy, John and Margit Tavits, "Beliefs and Voting Decisions: A Test of the Pivotal Voter Model," American Journal of Political Science, 2008, 52 (3), 603618.

Elinder, Mikael, Henrik Jordahl, and Panu Poutvaara, "Promises, Policies and Pocketbook Voting," European Economic Review, 2015, 75, 177-194.

Epple, Dennis N. and Richard E. Romano, "Public Provision of Private Goods," Journal of Political Economy, 1996, 104 (1), 57-84.

_, Thomas Romer, and Holger Sieg, "Interjurisdictional Sorting and Majority Rule: An Empirical Analysis," Econometrica, 2001, 69 (6), 1437-1465.

Fehr, Ernst and Klaus M. Schmidt, "A Theory of Fairness, Competition, and Cooperation," Quarterly Journal of Economics, 1999, 114 (3), 817-868. 
Feld, Lars P. and Gebhard Kirchgässner, "The Political Economy of Direct Legislation: Direct Democracy and Local Decision-Making," Economic Policy, 2001, 16 (33), 329-367.

- and John G. Matsusaka, "Budget Referendums and Government Spending: Evidence from Swiss Cantons," Journal of Public Economics, 2003, 87 (12), 27032724.

Fiorina, Morris P., "Economic Retrospective Voting in American National Elections: A Micro-Analysis," American Journal of Political Science, 1978, 22 (2), $426-443$.

Fischer, A. J., "A Further Experimental Study of Expressive Voting," Public Choice, 1996, 88, 171-184.

Fong, Christina, "Social Preferences, Self-Interest, and the Demand for Redistribution," Journal of Public Economics, 2001, 82 (2), 225-246.

Funk, Patricia, "Social Incentives and Voter Turnout: Evidence from the Swiss Mail Ballot System," Journal of the European Economic Association, 2010, 8 (5), 1077-1103.

- and Christina Gathmann, "Does Direct Democracy Reduce the Size of Government? New Evidence from Historical Data, 1890-2000," Economic Journal, 2011, 121 (557), 1252-1280.

Gerber, Alan S., Donald P. Green, and Christopher W. Larimer, "Social Pressure and Voter Turnout: Evidence from a Large-Scale Field Experiment," American Political Science Review, 2008, 102 (1), 33-48.

Hamlin, Alan and Colin Jennings, "Expressive Political Behaviour: Foundations, Scope and Implications," British Journal of Political Science, 2011, 41 (3), 645-670.

Harsanyi, John C., "Rule Utilitarianism, Rights, Obligations and the Theory of Rational Behavior," Theory and Decision, 1980, 12 (2), 115-133.

Hillman, Arye L., "Expressive Behavior in Economics and Politics," European Journal of Political Economy, 2010, 26 (4), 403-418.

_ , Kfir Metsuyanim, and Niklas Potrafke, "Democracy with Group Identity," European Journal of Political Economy, 2015, 40 (B), 274-287. 
Kahneman, Daniel and Amos Tversky, "Prospect Theory: An Analysis of Decision under Risk," Econometrica, 1979, 47 (2), 263-292.

Levitt, Steven D. and James M. Snyder, "The Impact of Federal Spending on House Election Outcomes," Journal of Political Economy, 1997, 105 (1), 30-53.

Lewis-Beck, Michael S. and Mary Stegmaier, "Economic Models of Voting," in Russel J. Dalton and Hans-Dieter Klingemann, eds., The Oxford Handbook of Political Behavior, 2007, pp. 518-537.

Lindbeck, Assar and Jörgen W. Weibull, "A Model of Political Equilibrium in a Representative Democracy," Journal of Public Economics, 1993, 51 (2), 195-209.

Manacorda, Marco, Edward Miguel, and Andrea Vigorito, "Government Transfers and Political Support," American Economic Journal: Applied Economics, 2011, 3 (3), 1-28.

Markus, Gregory B., "The Impact of Personal and National Economic Conditions on the Presidential Vote: A Pooled Cross-Sectional Analysis," American Journal of Political Science, 1988, 32 (1), 137-154.

Matsusaka, John G., "Fiscal Effects of the Voter Initiative: Evidence from the Last 30 Years," Journal of Political Economy, 1995, 103 (3), 587-623.

Meltzer, Allan H. and Scott F. Richard, "A Rational Theory of the Size of Government," Journal of Political Economy, 1981, 89 (5), 914-927.

Messer, Kent D., Gregory L. Poe, Daniel Rondeau, William D. Schulze, and Christian A. Vossler, "Social Preferences and Voting: An Exploration Using a Novel Preference Revealing Mechanism," Journal of Public Economics, 2010, 94 (3-4), 308-317.

Piketty, Thomas, "Social Mobility and Redistributive Politics," Quarterly Journal of Economics, 1995, 110 (3), 551-584.

Potrafke, Niklas, "Evidence on the Political Principal-Agent Problem from Voting on Public Finance for Concert Halls," Constitutional Political Economy, 2013, 24 (3), 215-238.

Riker, William H. and Peter C. Ordeshook, "A Theory of the Calculus of Voting," American Political Science Review, 1968, 62 (1), 25-42. 
Romer, Thomas, Howard Rosenthal, and Vincent G. Munley, "Economic Incentives and Political Institutions: Spending and Voting in School Budget Referenda," Journal of Public Economics, 1992, 49 (1), 1-33.

Rousseau, Jean-Jacques, On the Social Contract, Mineola, New York: Dover Publications, 2012 [1762].

Schumpeter, Joseph A., Capitalism, Socialism and Democracy, London, New York: Routledge, 1994 [1942].

Taylor, Curtis R. and Huseyin Yildirim, "A Unified Analysis of Rational Voting with Private Values and Group-specific Costs," Games and Economics Behavior, 2010, 70, 457-471.

Tiebout, Charles, "A Pure Theory of Local Expenditures," Journal of Political Economy, 1956, 64 (5), 416-424.

Tullock, Gordon, "The Charity of the Uncharitable," Western Economic Journal, 1971, 9 (4), 379-392.

Tyran, Jean-Robert, "Voting when Money and Morals Conflict: An Experimental Test of Expressive Voting," Journal of Public Economics, 2004, 88, 1645-1664.

- and Rupert Sausgruber, "A Little Fairness May Induce a lot of Redistribution in Democracy," European Economic Review, 2006, 50 (2), 469-485.

Vlachos, Jonas, "Who Wants Political Integration? Evidence from the Swedish EU-Membership Referendum," Journal of Public Economics, 2004, 88 (7-8), 15891604 . 